\title{
REAL SECOND ORDER FREENESS AND HAAR ORTHOGONAL MATRICES
}

\author{
JAMES A. $\operatorname{MINGO}^{(*)}$ AND MIHAI POPA $(*)(\ddagger)$
}

\begin{abstract}
We demonstrate the asymptotic real second order freeness of Haar distributed orthogonal matrices and an independent ensemble of random matrices. Our main result states that if we have two independent ensembles of random matrices with a real second order limit distribution and one of them is invariant under conjugation by an orthogonal matrix, then the two ensembles are asymptotically real second order free. This captures the known examples of asymptotic real second order freeness introduced by Redelmeier $\left[\mathrm{R}_{1}, \mathrm{R}_{2}\right]$.
\end{abstract}

\section{INTRODUCTION}

The large $N$ behaviour of random matrices has been actively studied since Wigner's celebrated semi-circle law was found in 1955, [W]. Subsequently in 1967 Marchenko and Pastur found the limit distribution for Wishart matrices [MP1], now called the Marchenko-Pastur distribution. The essential point of these discoveries is that for many ensembles of random matrices the description of the distribution of the eigenvalues gets much simpler in the large $N$ limit. Much subsequent work has been devoted to expanding and refining this work, see for example the recent book of Anderson, Guionnet, and Zeitouni [AGZ].

Another direction of research in random matrices deals with the interaction of independent ensembles of random matrices. In this direction one studies the limit eigenvalue distribution of sums and products of ensembles whose limit distributions are already known. The direction was discovered by Voiculescu in his work on free probability. In $\left[\nabla_{1}\right]$ and later in $\left[\nabla_{2}\right]$, Voiculescu showed that independent ensembles were asymptotically free if at least one was unitarily invariant. Recall that if two random variables are freely independent then there is

* Research supported by a Discovery Grant from the Natural Sciences and Engineering Research Council of Canada.

$(\ddagger)$ Research supported by the Natural Science Foundation of China Grant No. 11150110456, and the Romanian National Authority for Scientific Research, CNCS UEFISCDI, Project Number PN-II-ID-PCE-2011-3-0119. 
a universal rule for finding the mixed moments from the moments of the individual random variables. One does this either analytically by using the $R$ and $S$ transform, see [VDN], or combinatorially using free cumulants, see [NS].

In the last two decades the fluctuations of the eigenvalues have been studied both in the physics and the mathematics literate, see e.g. [AZ, BS, FMP, J, K, KKP]. In [MN] it was shown that the fluctuations of Wishart matrices could be analyzed using the non-crossing diagrams introduced in [S], but by using an annulus instead of a disc or line, see Figure 1, hence all the combinatorial techniques developed by Nica and Speicher [NS] could be brought to bear on the study of fluctuations. Thus motivated, second order freeness was introduced in [MS, MŚS] and later higher order freeness in [CMŚS.

The point of second and higher order freeness is that it enables one to do for fluctuation moment and higher order trace-moments what Voiculesu's first order freeness did for moments. In particular if two random variables are second order free and one knows the moments and the fluctuation moments of each variable then there is a universal rule for finding fluctuation moments of sums and products, see [MST].

In [CMŚS, MN, MS, MŚS the random matrices considered were either Hermitian or unitary. This left the question of how to deal with real symmetric and orthogonal matrices. On the first order level the techniques of Voiculescu were equally applicable to real and complex ensembles. However it was shown in $\left[\mathrm{R}_{1}, \mathrm{R}_{2}\right]$ that the universal rule found in [MS] needed to be modified for the real case; in particular the transpose of the various operators made an appearance. This led to a new kind of second order freeness, called real second order freeness in $\left[\mathrm{R}_{1}, \mathrm{R}_{2}\right]$.

The non-crossing diagrams introduced in [MN] had to augmented by diagrams in which the orientation of one of the circles was reversed. The operators on the reversed side get transposed. One can give a heuristic interpretation of this using maps on surfaces, see [LZ]. In the complex case we only work with orientable surfaces and in the real case we also have to also deal with non-orientable surfaces. So we imagine that our surfaces are marked our operators and the graphs tell us how they get multiplied, see Figure 5. Wherever we put an operator on the front side of the surface, we put its transpose on the back. The non-orientability of the surface means that we can cross from font to back and see the transposed operators, something that we cannot do in the complex case.

The main result of this paper, Theorem 54, asserts that if $\left\{A_{i}\right\}_{i}$ and $\left\{B_{j}\right\}_{j}$ are independent ensembles of random matrices and if at least one 
of them is invariant under conjugation by an orthogonal matrix then the ensembles are asymptotically real second order free. The proof of this theorem occupies nearly the whole paper. This theorem is the orthogonal version of a theorem in [MŚS], where we assumed that one of the ensembles is invariant under conjugation by an unitary matrix. While the statements of the two theorems are similar the proofs follow quite different paths. In MŚs the asymptotics of the cumulants of the unitary Weingarten function, from [C], were heavily used. In this paper we only need the multiplicitivity of the leading order of the orthogonal Weingarten function, see [CS]. We work with centred elements and this obviates the need to work with the cumulants of the Weingarten function.

Illustrative examples. Let us conclude this introduction with some examples. Suppose that $A_{1}, A_{2}, A_{3}, A_{4}$ are $d \times d$ deterministic matrices and $O$ is a $d \times d$ Haar distributed random orthogonal matrix and $U$ be a $d \times d$ Haar distributed random unitary matrix. From $\mathrm{MŚS}$, Prop. 3.4] we have

$$
\begin{gathered}
\mathrm{E}\left(\operatorname{Tr}\left(U A_{1} U^{-1} A_{2}\right)\right)=d^{-1} \operatorname{Tr}\left(A_{1}\right) \operatorname{Tr}\left(A_{2}\right) \text { and } \\
\mathrm{E}\left(\operatorname{Tr}\left(U A_{1} U A_{2}\right)\right)=0 .
\end{gathered}
$$

According to Proposition 12

$$
\begin{gathered}
\mathrm{E}\left(\operatorname{Tr}\left(O A_{1} O^{-1} A_{2}\right)\right)=d^{-1} \operatorname{Tr}\left(A_{1}\right) \operatorname{Tr}\left(A_{2}\right) \text { and } \\
\mathrm{E}\left(\operatorname{Tr}\left(O A_{1} O A_{2}\right)\right)=d^{-1} \operatorname{Tr}\left(A_{1} A_{2}^{t}\right) .
\end{gathered}
$$

So we already see a bit a difference between the orthogonal and unitary cases; namely the appearance of transposes in lower order terms. When we consider covariances we see more differences. First in the unitary case we have

$$
\begin{gathered}
\operatorname{cov}\left(\operatorname{Tr}\left(U A_{1} U^{-1} A_{2}\right), \operatorname{Tr}\left(U A_{3} U^{-1} A_{4}\right)\right) \\
=\frac{d^{-4}}{1-d^{-2}} \operatorname{Tr}\left(A_{1}\right) \operatorname{Tr}\left(A_{2}\right) \operatorname{Tr}\left(A_{3}\right) \operatorname{Tr}\left(A_{4}\right) \\
\quad+\frac{d^{-2}}{1-d^{-2}} \operatorname{Tr}\left(A_{1} A_{3}\right) \operatorname{Tr}\left(A_{2} A_{4}\right) \\
-\frac{d^{-3}}{1-d^{-2}}\left\{\operatorname{Tr}\left(A_{1} A_{3}\right) \operatorname{Tr}\left(A_{2}\right) \operatorname{Tr}\left(A_{4}\right)+\operatorname{Tr}\left(A_{1}\right) \operatorname{Tr}\left(A_{2} A_{4}\right) \operatorname{Tr}\left(A_{3}\right)\right\} .
\end{gathered}
$$

Now in the orthogonal case we have

$$
\begin{array}{r}
\left(1+d^{-1}-2 d^{-2}\right) \operatorname{cov}\left(\operatorname{Tr}\left(O A_{1} O^{-1} A_{2}\right), \operatorname{Tr}\left(O A_{3} O^{-1} A_{4}\right)\right) \\
=d^{-4}\left\{\operatorname{Tr}\left(A_{1}\right) \operatorname{Tr}\left(A_{2}\right) \operatorname{Tr}\left(A_{3}\right) \operatorname{Tr}\left(A_{4}\right)\right.
\end{array}
$$




$$
\begin{gathered}
\left.+\operatorname{Tr}\left(A_{1}\right) \operatorname{Tr}\left(A_{2}\right) \operatorname{Tr}\left(A_{3}^{t}\right) \operatorname{Tr}\left(A_{4}^{t}\right)\right\} \\
-d^{-3}\left\{\operatorname{Tr}\left(A_{1} A_{3}\right) \operatorname{Tr}\left(A_{2}\right) \operatorname{Tr}\left(A_{4}\right)+\operatorname{Tr}\left(A_{1} A_{3}^{t}\right) \operatorname{Tr}\left(A_{2}\right) \operatorname{Tr}\left(A_{4}^{t}\right)\right. \\
\left.+\operatorname{Tr}\left(A_{1}\right) \operatorname{Tr}\left(A_{2} A_{4}\right) \operatorname{Tr}\left(A_{3}\right)+\operatorname{Tr}\left(A_{1}\right) \operatorname{Tr}\left(A_{2} A_{4}^{t}\right) \operatorname{Tr}\left(A_{3}^{t}\right)\right\} \\
+\left(d^{-2}+d^{-3}\right)\left\{\operatorname{Tr}\left(A_{1} A_{3}\right) \operatorname{Tr}\left(A_{2} A_{4}\right)+\operatorname{Tr}\left(A_{1} A_{3}^{t}\right) \operatorname{Tr}\left(A_{2} A_{4}^{t}\right)\right\} \\
-d^{-3}\left\{\operatorname{Tr}\left(A_{1} A_{3}^{t}\right) \operatorname{Tr}\left(A_{2} A_{4}\right)+\operatorname{Tr}\left(A_{1} A_{3}\right) \operatorname{Tr}\left(A_{2} A_{4}^{t}\right)\right\} .
\end{gathered}
$$

Note the similarity to the unitary case except that each term of leading order appears twice-once with no transposes and once with transposes on $A_{3}$ and $A_{4}$. Moreover when the $A_{i}$ 's are centred, i.e. $\operatorname{Tr}\left(A_{i}\right)=0$, the only remaining terms are $\operatorname{Tr}\left(A_{1} A_{3}\right) \operatorname{Tr}\left(A_{2} A_{4}\right)$ and $\operatorname{Tr}\left(A_{1} A_{3}^{t}\right) \operatorname{Tr}\left(A_{2} A_{4}^{t}\right)$. These terms correspond to spoke diagrams which are discussed in the next section, see Figure 2. By working with centred elements the number of terms is significantly reduced, it is in this way that we can skip the calculations requiring the cumulants of the Weingarten function.

The Organization of the Paper. In section 2 we review the definitions of non-crossing partitions. In section 3 we use the Weingarten function of [CS] to compute the trace of a product of orthogonal matrices and independent random matrices. This is how the calculations in the examples above were done. In section 4 we prove two important lemmas on a special kind of non-crossing partition called a spoke diagram. These are the only diagrams that survive in the large $d$ limit. In section 5 we recall the notions of second order freeness from $\left[\mathrm{R}_{1}, \mathrm{R}_{2}\right]$ and prove that real second order freeness satisfies an associative law. In section 6 we prove that Haar distributed orthogonal matrices and an independent ensemble are first order free. That this could be done was already suggested by Voiculescu in $\left[\mathrm{V}_{1}\right]$ some twenty years ago and was later proved in [CS, Thm. 5.2]. In section 7 we show that the fluctuation moments of Haar distributed orthogonal matrices and an independent ensemble of random matrices satisfy the universal rule required for second order freeness. In section 8 we show that the third and higher cumulants of traces of products of Haar distributed orthogonal matrices and an independent ensemble of random matrices satisfy the final condition for asymptotic real second order freeness. This completes the proof of their asymptotic real second order freeness. In section 9 we use this result to obtain all our other results on asymptotic real second order freeness. In section 10 we present some concluding remarks and indications of future work. 


\section{NON-CROSSING DIAGRAMS AND PAIRINGS}

Central to the combinatorial approach to freeness is the idea of a noncrossing partition. A partition of $[n]$ is non-crossing is one in which the blocks can be drawn in a non-crossing way; see the left half of Figure 1. For second order freeness we need non-crossing annular partitions. This means we can draw the blocks on an annulus in a non-crossing way; see the right half of Figure 1. In the case of second order freeness additional information about the partitions is needed, namely the order in which they visit the points. For this reason we regard our partitions as permutations by interpreting the blocks of the partition as cycles in the cycle decomposition of the corresponding permutation.

Notation 1. For any integer $n \geq 1$, let $[n]=\{1,2,3, \ldots, n\}$. Let $\mathcal{P}(n)$ be the set of all partitions of $[n]$. For any partition $\pi$ of $[n]$ let $\#(\pi)$ denote the number of blocks of $\pi$, and $|\pi|=n-\#(\pi)$. The set $\mathcal{P}(n)$ is a partially ordered set in which $\pi \leq \sigma$ means every block of $\pi$ is contained in some block of $\sigma$. With this order $\mathcal{P}(n)$ is partially ordered set and is in fact a lattice. We denoted the join of two partitions $\pi$ and $\sigma$ by $\pi \vee \sigma$.

Given a permutation it can be difficult to decide if there is a noncrossing way of drawing its cycles, however there is an algebraic way to see if such a diagram exists. Let $\gamma=(1, \ldots, m)(m+1, \ldots, m+n)$ and let $\pi$ be a permutation of $[m+n]$ and denote by $\langle\pi, \gamma\rangle$ the subgroup of $S_{n}$ generated by $\pi$ and $\gamma$. If the subgroup $\langle\pi, \gamma\rangle$ acts transitively on $[m+n]$ then we have that $\pi$ is non-crossing if and only if

$$
\#(\pi)+\#\left(\pi^{-1} \gamma\right)=m+n .
$$

Note that the condition that $\langle\pi, \gamma\rangle$ act transitively is the same as requiring that there is at least one cycle of $\pi$ that contains points in both cycles of $\gamma$. When this happen we shall say that $\pi$ connects the cycles of $\gamma$

We can extend this to the case of $\gamma$ having any number of cycles. Let $\pi$ and $\gamma$ be permutations of $[n]$. Let $k$ be the number of orbits of $\langle\pi, \gamma\rangle$. Then

$$
\#(\pi)+\#\left(\pi^{-1} \gamma\right)+\#(\gamma) \leq n+2 k
$$

with equality only if $\pi$ is non-crossing with respect to $\gamma$, see e.g. [MN, Remark 2.11].

In the case of real second order freeness we require an additional set of non-crossing diagrams, we call these reversed non-crossing annular permutations. If we let $\gamma^{\prime}=(1, \ldots, m)(m+n, m+n-1, \ldots, m+2, m+1)$ then we say that a permutation $\pi \in S_{m+n}$ is a reversed non-crossing 

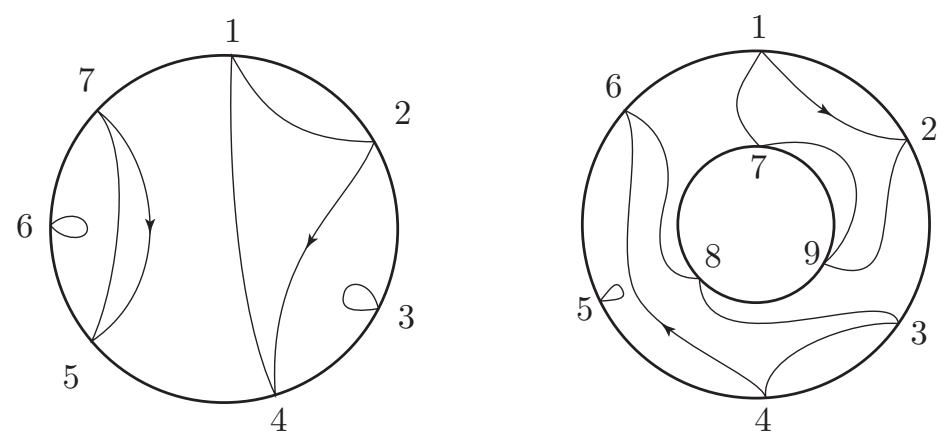

Figure 1. On the left we have the non-crossing disc permutation $(1,2,4)(3)(5,7)(6)$. On the right we have the non-crossing annular permutation $(1,2,9,7)$ $(3,4,6,8)(5)$.

permutation of a $(m, n)$-annulus if

$$
\#(\pi)+\#\left(\pi^{-1} \gamma^{\prime}\right)=m+n .
$$

Notice that this is the same condition as in Equation (1) but $\gamma$ is replaced with $\gamma^{\prime}$. Graphically, this corresponds to using the same orientation for labelling the points on each circle; see the right hand side of Figure 2,

A special kind of a non-crossing annular permutation that we shall make use of is that of a spoke diagram, see Figure 2. Recall that a pairing of $[n]$ is a partition in which each block has two elements. We usually regard a pairing as a permutation, by considering each block to be a cycle with two elements. By a standard spoke diagram we mean a non-crossing pairing of an $(m, n)$-annulus in which all pairs connect the two circles. Note that means that $m=n$ and there is $l$ such that $m+1 \leq l \leq 2 m$ such that every cycle of $p$ is of the form $\left(k, \gamma^{-k}(l)\right)$ for $1 \leq k \leq m$.

By a reversed spoke diagram we mean a reversed non-crossing annular pairing in which all blocks connect the two circles; see Figure 2. By a spoke diagram we mean either a standard or reversed spoke diagram. See Figure 2. Note that means that $m=n$ and there is $l$ such that $m+1 \leq l \leq 2 m$ such that every cycle of $p$ is of the form $\left(k, \gamma^{k}(l)\right)$ for $1 \leq k \leq m$.

We denote by $\mathcal{P}_{2}(n)$ the pairings of $[n]$. If $p$ is a pairing of $[n]$ and $(r, s)$ is a cycle of $p$ we shall denote this by $(r, s) \in p$. We denote by $\mathrm{Sp}^{+}(\mathrm{m})$ the set of all standard spoke diagrams and by $\mathrm{Sp}^{-}(\mathrm{m})$ the set of all reversed spoke diagrams. 

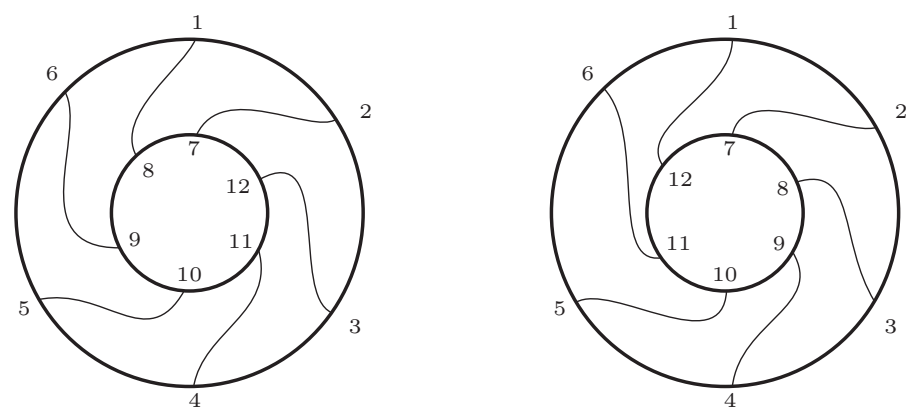

Figure 2. On the left we have a non-crossing pairing of a $(6,6)$-annulus in which all blocks connect the two circles, i.e. a standard spoke diagram. Note that the two circles have opposite orientations. In the figure on the right we have a reversed non-crossing pairing of a $(6,6)$ annulus. i.e. a reversed spoke diagram. Note that the two circles having the same orientation.

Given a permutation $\pi \in S_{n}$, we shall frequently consider the cycles of $\pi$ as a partition of $[n]$. This map $S_{n} \longrightarrow \mathcal{P}(n)$ forgets the order of elements in a cycle and so is not a bijection. Conversely given a partition $\pi \in \mathcal{P}(n)$ we put the elements of each block into increasing order and consider this a permutation. Restricted to pairings this is a bijection.

\section{The Trace of a Product}

Given a permutation $\sigma \in S_{n}$ and $d \times d$ matrices $A_{1}, \ldots, A_{n}$ we let $a_{p, q}^{(i)}$ be the $(p, q)$-entry of $A_{i}$ and

$$
\operatorname{Tr}_{\sigma}\left(A_{1}, \ldots, A_{n}\right)=\sum_{i_{1}, \ldots, i_{n}=1}^{d} a_{i_{1} i_{\sigma(1)}}^{(1)} \cdots a_{i_{n} i_{\sigma(n)}}^{(n)} .
$$

This expression can also be written as a product of traces as follows. Write $\sigma=c_{1} \cdots c_{k}$ in cycle form. If $c=\left(i_{1}, \ldots, i_{r}\right)$ is a cycle of $\sigma$ we let $\operatorname{Tr}_{c}\left(A_{1}, \ldots, A_{n}\right)=\operatorname{Tr}\left(A_{i_{1}} \cdots A_{i_{r}}\right)$. Then

$$
\operatorname{Tr}_{\sigma}\left(A_{1}, \ldots, A_{n}\right)=\prod_{i=1}^{k} \operatorname{Tr}_{c_{i}}\left(A_{1}, \ldots, A_{n}\right) .
$$

Let $O=\left(o_{i j}\right)$ be a $d \times d$ Haar distributed random orthogonal matrix and $\left\{Y_{1}, \ldots, Y_{n}\right\}$ be $d \times d$ random matrices whose entries have moments of all orders. Let $\gamma \in S_{n}$ be a permutation, and let $\epsilon_{1}, \epsilon_{2}, \ldots, \epsilon_{n} \in$ 
$\{-1,1\}$. In this section we wish to find a simple expression for

$$
\mathrm{E}\left(\operatorname{Tr}_{\gamma}\left(O^{\epsilon_{1}} Y_{1}, \ldots, O^{\epsilon_{n}} Y_{n}\right)\right)
$$

We shall use the Weingarten function introduced by Collins and Śniady [CS]. The Weingarten expresses the expectation $\mathrm{E}\left(o_{i_{1} i_{-1}} \cdots\right.$ $o_{i_{n} i_{-n}}$ ) as a sum over pairings of $[n]$. The first question we need to address is, for two pairings $p$ and $q$, the relationship between the cycles of $p q$ and the blocks of $p \vee q$. See Figure 3. This is a standard fact; for the reader's convenience and to establish our notation we give a proof.

Lemma 2. Let $p, q \in \mathcal{P}_{2}(n)$ be pairings and $\left(i_{1}, i_{2}, \ldots, i_{k}\right)$ a cycle of $p q$. Let $j_{r}=q\left(i_{r}\right)$. Then $\left(j_{k}, j_{k-1}, \ldots, j_{1}\right)$ is also a cycle of $p q$, and these two cycles are distinct; $\left\{i, \ldots, i_{k}, j_{1}, \ldots, j_{k}\right\}$ is a block of $p \vee q$ and all are of this form; $2 \#(p \vee q)=\#(p q)$.

Proof. We have $p q\left(i_{r}\right)=i_{r+1}$, thus $j_{r}=q\left(i_{r}\right)=p\left(i_{r+1}\right)$. Hence $p q\left(j_{r+1}\right)=p\left(q\left(q\left(i_{r+1}\right)\right)\right)=p\left(i_{r+1}\right)=j_{r}$. If $\left\{i_{1}, \ldots, i_{k}\right\}$ and $\left\{j_{1}, \ldots, j_{k}\right\}$ were to have a non-empty intersection then, for some $n, q(p q)^{n}$ would have a fixed point, but this would in turn imply that either $p$ or $q$ had a fixed point, which is impossible. Since $\left\{q\left(i_{r}\right)\right\}_{r}=\left\{j_{s}\right\}_{s}$ and $\left\{p\left(j_{s}\right)\right\}_{s}=\left\{i_{r}\right\}_{r},\left\{i, \ldots, i_{k}, j_{1}, \ldots, j_{k}\right\}$ must be a block of $p \vee q$. Since every point of $[n]$ is in some cycle of $p q$, all blocks must be of this form. Since every block of $p \vee q$ is the union of two cycles of $p q$, we have $2 \#(p \vee q)=\#(p q)$.

Notation 3. Let $[-n]=\{-n,-n+1, \ldots,-2,-1\}$ and $[ \pm n]=[-n] \cup$ $[n]$. Let $\delta$ be the permutation of $[ \pm n]$ which sends $k$ to $-k$ for $k \in[ \pm n]$. Since each cycle of $\delta$ is of the form $(k,-k)$, we shall also regard $\delta$ as a pairing of $[ \pm n]$. If $\epsilon \in \mathbb{Z}_{2}^{n}=\{-1,1\}^{n}$, let $\delta_{\epsilon}$ denote the permutation of $[ \pm n]$ given by $k \mapsto \epsilon_{|k|} k$.

Given $\pi$ a permutation on $[n]$ we shall regard $\pi$ also a permutation of $[ \pm n]$ where for $1 \leq k \leq n$, we let $\pi(-k)=-k$. Let $\gamma$ be the permutation of $[n]$ with the one cycle $(1,2,3, \ldots, n)$, but following the convention mentioned above we also have $\gamma(-k)=-k$ for $1 \leq k \leq n$.

Lemma 4. Let $p, q \in \mathcal{P}_{2}(n)$ be pairings then $\#(p q)=\#(p \delta q)$.

Proof. Note that for $1 \leq k \leq n$ we have $p \delta q(k)<0$ and $p \delta q(-k)>0$. Thus the elements in an orbit of $p \delta q$ always alternate in sign. Moreover $(p \delta q)^{2}=p q$. Hence the positive elements of a cycle of $p \delta q$ form a cycle of $p q$. Conversely let $\left(i_{1}, i_{2}, \ldots, i_{r}\right)$ be a cycle of $p q$. Then $\left(i_{1},-q\left(i_{1}\right), i_{2},-q\left(i_{2}\right), \ldots, i_{r},-q\left(i_{r}\right)\right)$ is a cycle of $p \delta q$. This establishes a bijection between the cycles of $p \delta q$ and the cycles of $p q$. 


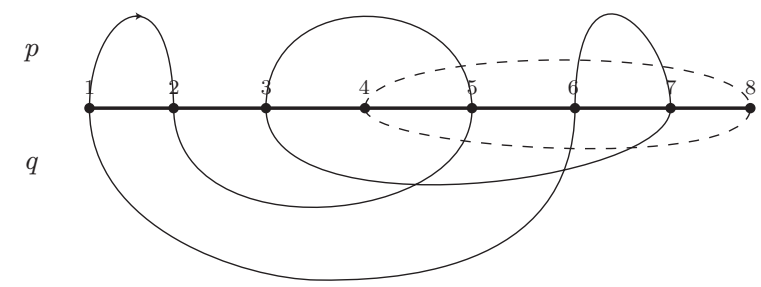

Figure 3. In this example $n=8, p=(1,2)(3,5)$ $(4,8)(6,7)$, and $q=(1,6)(2,5)(3,7)(4,8)$. Then $p q=$ $(1,7,5)(2,3,6)(4)(8)$ and $p \vee q=\{(1,2,3,5,6,7)(4,8)\}$.

The pairings of $[ \pm n]$ shall be denoted $\mathcal{P}_{2}( \pm n)$. For a pairing $p \in$ $\mathcal{P}_{2}( \pm n)$, and a $2 n$-tuple $i=\left(i_{1}, i_{-1}, \ldots, i_{n}, i_{-n}\right)$ we write $i=i \circ p$ to mean that whenever $p(r)=s$ we have $i_{r}=i_{s}$. For a $d \times d$ matrix $A$ let $A^{(-1)}=A^{t}$, the transpose of $A$, and $A^{(1)}=A$. For $\eta=\left(\eta_{1}, \eta_{2}, \ldots, \eta_{n}\right) \in$ $\mathbb{Z}_{2}^{n}$ and $\pi \in S_{n}$, let $\operatorname{Tr}_{(\pi, \eta)}\left(A_{1}, \ldots, A_{n}\right)=\operatorname{Tr}_{\pi}\left(A_{1}^{\left(\eta_{1}\right)}, \ldots, A_{n}^{\left(\eta_{n}\right)}\right)$

Lemma 5. Let $p \in \mathcal{P}_{2}( \pm n)$. The there is $\pi \in S_{n}$ and $\eta \in \mathbb{Z}_{2}^{n}$ such that

$$
\sum_{\substack{i=i_{1}, \ldots, i_{n}, i_{-n}=1 \\ i=i \circ p}}^{d} a_{i_{1} i_{-1}}^{(1)} a_{i_{2} i_{-2}}^{(2)} \cdots a_{i_{k} i_{-k}}^{(k)} \cdots a_{i_{n} i_{-n}}^{(n)}=\operatorname{Tr}_{\pi}\left(A_{1}^{\left(\eta_{1}\right)}, \ldots, A_{n}^{\left(\eta_{n}\right)}\right)
$$

Proof. We saw that the cycle decomposition of $p \delta$ may be written $c_{1} c_{1}{ }^{\prime} \cdots c_{s} c_{s}{ }^{\prime}$ where $c_{i}{ }^{\prime}=\delta c_{i}^{-1} \delta$. It is arbitrary which of the pair $\left\{c_{i}, c_{i}{ }^{\prime}\right\}$ is called $c_{i}$ and which $c_{i}{ }^{\prime}$.

For each $i$, choose a representative of each pair $\left\{c_{i}, c_{i}{ }^{\prime}\right\}$, say $c_{1}, c_{2}, \ldots$, $c_{s}$. For each $i$ we construct a cycle $\tilde{c}_{i}$ as follows. Suppose $c_{i}=$ $\left(l_{1}, \ldots, l_{r}\right)$. Let $\tilde{c}_{i}=\left(j_{1}, j_{2}, \ldots, j_{r}\right)$ where

$$
j_{k}=\left\{\begin{array}{ll}
-l_{k} & l_{k}<0 \\
l_{k} & l_{k}>0
\end{array} \text { and } \eta_{j_{k}}=\left\{\begin{array}{ll}
-1 & l_{k}<0 \\
1 & l_{k}>0
\end{array} .\right.\right.
$$

Note that $j_{k}=\eta_{j_{k}} l_{k}=\left|l_{k}\right|$. Then we let $\pi=\tilde{c}_{1} \cdots \tilde{c}_{s}$ and $\eta=$ $\left(\eta_{1}, \ldots, \eta_{n}\right)$.

We denote the $(m, n)$ entry of $A_{i}^{\left(\eta_{i}\right)}$ by $a_{m, n}^{\left(i, \eta_{i}\right)}$. Let $\left(l_{1}, \ldots, l_{r}\right)$ be a cycle of $p \delta$. Let $\left(j_{1}, \ldots, j_{1}\right)$ and $\left(\eta_{1}, \ldots, \eta_{n}\right)$ be as above i.e. $j_{k}=\left|l_{k}\right|$ and $\eta_{j_{k}}=l_{k} /\left|l_{k}\right|$. Then

$$
a_{i_{j_{k}} i_{-j_{k}}}^{\left(j_{j_{k}}\right)}=\left\{\begin{array}{ll}
a_{i_{j_{k}} i_{-j_{k}}}^{\left(j_{k}, \eta_{j_{k}}\right)} & \text { if } \eta_{j_{k}}=1 \\
a_{i_{-j_{k}} i_{j_{k}}}^{\left(j_{k}, \eta_{j_{k}}\right)} & \text { if } \eta_{j_{k}}=-1
\end{array}\right\}=a_{\left.i_{l_{k}} i_{-l_{k}} i_{j_{k}}\right)}^{\left(j_{k}, \eta_{j_{k}}\right)}
$$

Thus

$$
a_{i_{j_{1}} i_{-j_{1}}}^{\left(j_{1}\right)} \cdots a_{i_{j_{r}} i_{-j_{r}}}^{\left(j_{r}\right)}=a_{i_{l_{1}} i_{-l_{1}}}^{\left(j_{1}, \eta_{j_{1}}\right)} \cdots a_{i_{l_{r}} i_{-i_{r}}}^{\left(j_{j_{2}, \eta_{j_{r}}}\right)}
$$


Note that $i_{-l_{k}}=i\left(\delta\left(l_{k}\right)\right)=i\left(p \delta\left(l_{k}\right)\right)=i\left(l_{k+1}\right)=i_{l_{k+1}}$, as $i=i \circ p$. Thus

$$
\begin{aligned}
& \sum_{\substack{i_{1}, \ldots, i_{-n}=1 \\
i=i \circ p}}^{d} a_{i_{1} i_{-1}}^{(1)} a_{i_{2} i_{-2}}^{(2)} \cdots a_{i_{k} i_{-k}}^{(k)} \cdots a_{i_{n} i_{-n}}^{(n)} \\
= & \sum_{\substack{i_{1}, \ldots, i_{-n}=1 \\
i=i \circ p}}^{d} \prod_{\substack{\tilde{c} \in \pi \\
c=\left(j_{1}, \ldots, j_{r}\right)}} a_{i_{j_{1}} i_{-j_{1}}}^{\left(j_{1}\right)} \cdots a_{i_{j_{r}} i_{-j_{r}}}^{\left(j_{r}\right)} \\
= & \sum_{\substack{i_{1}, \ldots, i_{2 n}=1 \\
i=i \circ p}}^{d} \prod_{\substack{\tilde{c} \in \pi \\
\tilde{c}=\left(j_{1}, \ldots, j_{r}\right)}} a_{i_{l_{1}} i_{-l_{1}}}^{\left(j_{1}, \eta_{j_{1}}\right)} \cdots a_{i_{l_{r}} i_{-i_{r}}}^{\left(j_{r}, \eta_{j_{r}}\right)} \\
= & \prod_{\substack{\tilde{c} \in \pi \\
\tilde{c}=\left(j_{1}, \ldots, j_{r}\right)}}^{\operatorname{Tr}\left(A_{j_{1}}^{\left(\eta_{j_{1}}\right)} \cdots A_{j_{r}}^{\left(\eta_{j_{r}}\right)}\right)} \\
= & \operatorname{Tr}_{\pi}\left(A_{1}^{\left(\eta_{1}\right)}, \ldots, A_{n}^{\left(\eta_{n}\right)}\right)
\end{aligned}
$$

Remark 6 . The pair $(\pi, \eta)$ constructed in Lemma 5 is not unique; however since

$$
\operatorname{Tr}\left(A_{j_{1}}^{\left(\eta_{j_{1}}\right)} \cdots A_{j_{r}}^{\left(\eta_{j_{r}}\right)}\right)=\operatorname{Tr}\left(A_{j_{r}}^{\left(-\eta_{j_{r}}\right)} \cdots A_{j_{1}}^{\left(-\eta_{j_{1}}\right)}\right)
$$

the value of $\operatorname{Tr}_{\pi}\left(A_{1}^{\left(\eta_{1}\right)}, \ldots, A_{n}^{\left(\eta_{n}\right)}\right)$ is independent of the choices made.

Notation 7. Let $\mathbb{C}\left[\mathcal{P}_{2}(n)\right]$ be the inner product vector space with orthonormal basis $\mathcal{P}_{2}(n)$. For an integer $d \geq n$, define $\varphi: \mathbb{C}\left[\mathcal{P}_{2}(n)\right] \longrightarrow$ $\mathbb{C}\left[\mathcal{P}_{2}(n)\right]$ by

$$
\langle\varphi(p), q\rangle=d^{\#(p \vee q)}
$$

In [CS, §3], Collins and Śniady showed that $\varphi$ is an invertible linear transformation and denoted its inverse $\mathrm{Wg}$, the orthogonal Weingarten function. From the construction, $\langle\mathrm{Wg}(p), q\rangle$ is always a rational function of $d$. Collins and Sniady showed [CS, Thm. 3.13] that given $p, q \in \mathcal{P}_{2}(n)$ if we expand in power series in $d^{-1}$ then we have

$$
\langle\mathrm{Wg}(p), q\rangle=\mathrm{O}\left(d^{-n+\#(p \vee q)}\right) .
$$

Remark 8. It was shown in [CS] that the coefficient of $d^{-n+\#(p \vee q)}$ can be written as a product of signed Catalan numbers. Indeed, write $p q=\rho q \rho^{-1} q$ and factor $\rho$ into a product of cycles $c_{1} \cdots c_{k}$. Let $C_{m}$ be the $m^{t h}$ Catalan number $\frac{1}{m+1}\left(\begin{array}{c}2 m \\ m\end{array}\right)$. Then the coefficient of $d^{-n+\#(p \vee q)}$ is

$$
(-1)^{r_{1}-1} C_{r_{1}-1} \cdots(-1)^{r_{k}-1} C_{r_{k}-1}
$$


where the $i^{\text {th }}$ cycle $c_{i}$ has $r_{i}$ elements.

The reason for introducing $\mathrm{Wg}$ is its use in computing matrix expectations. For pairings $p, q \in \mathcal{P}_{2}(n), p \delta q \delta$ is a pairing of $[ \pm n]$. For a pairing $r$ of $[ \pm n]$ and $i_{1}, i_{-1}, \ldots, i_{n}, i_{-n} \in[d]$ we let $\delta_{r}^{i}=1$ if $i_{s}=i_{t}$ whenever $(s, t)$ is a pair of $r$ and 0 otherwise.

Theorem 9 ([CS, Cor. 3.4]). When $n$ is even

$$
\mathrm{E}\left(o_{i_{1} i_{-1}} \cdots o_{i_{n} i_{-n}}\right)=\sum_{p, q \in \mathcal{P}_{2}(n)}\langle\mathrm{Wg}(p), q\rangle \delta_{p \delta q \delta}^{i} .
$$

When $n$ is odd, $\mathrm{E}\left(o_{i_{1} i_{-1}} \cdots o_{i_{n} i_{-n}}\right)=0$.

Corollary 10. Let $O$ be a $d \times d$ Haar distributed orthogonal matrix and $m$ a non-zero integer. Then

$$
\lim _{d \rightarrow \infty} \mathrm{E}\left(\operatorname{tr}\left(O^{m}\right)\right)=0 .
$$

Proof. Let $\gamma \in S_{m}$ be the permutation with the one cycle $(1,2,3, \ldots$, $m)$. If $m$ is odd then $\mathrm{E}\left(\operatorname{Tr}\left(O^{m}\right)\right)=0$. So suppose that $m$ is even. First let us consider

$$
\begin{aligned}
\mathrm{E}\left(\operatorname{Tr}\left(O^{m}\right)\right) & =\sum_{i_{1}, \ldots, i_{m}=1}^{d} \mathrm{E}\left(o_{i_{1} i_{\gamma(1)}} \cdots o_{i_{n} i_{\gamma(n)}}\right) \\
& =\sum_{i_{1}, \ldots, i_{m}} \sum_{p, q \in \mathcal{P}_{2}(m)}\langle\mathrm{Wg}(p), q\rangle \delta_{p}^{i} \delta_{q}^{i \gamma},
\end{aligned}
$$

where $i \gamma$ is the $m$-tuple $\left(i_{\gamma(1)}, \ldots, i_{\gamma(m)}\right)$. Now $\delta_{q}^{i \gamma}=\delta_{\gamma q \gamma^{-1}}^{i}$. Thus $\delta_{p}^{i} \delta_{q}^{i \gamma}=1$ only when $i$ is constant on the blocks of $p \vee \gamma q \gamma^{-1}$. Hence

$$
\mathrm{E}\left(\operatorname{Tr}\left(O^{m}\right)\right)=\sum_{p, q \in \mathcal{P}_{2}(m)}\langle\mathrm{Wg}(p), q\rangle d^{\#\left(p \vee \gamma q \gamma^{-1}\right)} .
$$

Thus $\mathrm{E}\left(\operatorname{Tr}\left(O^{m}\right)\right)=\mathrm{O}\left(d^{-m+\#(p \vee q)+\#\left(p \vee \gamma q \gamma^{-1}\right)}\right)$. But $-m+\#(p \vee q)+$ $\#\left(p \vee \gamma q \gamma^{-1}\right) \leq 0$. Hence $\lim _{d \rightarrow \infty} \mathrm{E}\left(\operatorname{tr}\left(O^{m}\right)\right)=0$.

Notation 11. Let $\gamma \in S_{n}$ be a permutation of $[n]$ but, as in Notation 3. considered as a permutation of $[ \pm n]$ by setting $\gamma(-k)=-k$ for $1 \leq k \leq n$. Given $\epsilon \in \mathbb{Z}_{2}^{n}$ and $p, q \in \mathcal{P}_{2}(n)$ we consider the pairing of $[ \pm n]$ given by $p \cdot{ }_{\epsilon} q=(\gamma \delta)^{-1} \delta_{\epsilon} p \delta q \delta \delta_{\epsilon}(\gamma \delta)$ of $[ \pm n]$. By Lemma 5 there is a permutation $\pi_{p \cdot_{\epsilon} q} \in S_{n}$ and $\eta_{p \cdot{ }_{\epsilon} q} \in \mathbb{Z}_{2}^{n}$ such that

$$
\sum_{\substack{i_{1}, i_{-1}, \ldots, i_{n}, i_{-n}=1 \\ i=i \circ p \cdot \epsilon q}}^{d} a_{i_{1} i_{-1}}^{(1)} a_{i_{2} i_{-2}}^{(2)} \cdots a_{i_{k} i_{-k}}^{(k)} \cdots a_{i_{n} i_{-n}}^{(n)}=\operatorname{Tr}_{\left(\pi_{p \cdot \epsilon q}, \eta_{p \cdot \epsilon q}\right)}\left(A_{1}, \ldots, A_{n}\right) .
$$


Note that $p$ is a pairing of $[n], \delta q \delta$ is a pairing of $[-n]$ and so $p \delta q \delta$ is a pairing of $[ \pm n]$. If we adopt the notation $\gamma_{-}=\delta \gamma \delta$ then $\left(p \cdot{ }_{\epsilon} q\right) \delta=$ $\gamma_{-}^{-1} \delta_{\epsilon} q \delta p \delta_{\epsilon} \gamma$. Recall from the proof of Lemma 5 that $\pi_{p \cdot{ }_{\epsilon} q}$ was obtained by writing $\left(p \cdot{ }_{\epsilon} q\right) \delta$ as a product of cycles and taking one cycle of each pair $\left\{c, c^{\prime}\right\}$. After this choice has been made $\eta_{p \cdot{ }_{\epsilon} q}$ records the position of the minus signs.

Proposition 12. Let $O$ be a Haar distributed $d \times d$ random orthogonal matrix and $\left\{Y_{1}, \ldots, Y_{n}\right\} d \times d$ random matrices which are independent from $O$ and whose entries have moments of all orders. Let $\gamma \in S_{n}$, $\epsilon \in \mathbb{Z}_{2}^{n}$ and suppose $d \geq n$.

$$
\begin{aligned}
& \mathrm{E}\left(\operatorname{Tr}_{\gamma}\left(O^{\epsilon_{1}} Y_{1}, \ldots, O^{\epsilon_{n}} Y_{n}\right)\right) \\
& \quad=\sum_{p, q \in \mathcal{P}_{2}(n)}\langle\mathrm{Wg}(p), q\rangle \mathrm{E}\left(\operatorname{Tr}_{\left(\pi_{p \cdot \epsilon q}, \eta_{p \cdot \epsilon q}\right)}\left(Y_{1}, \cdots, Y_{n}\right)\right)
\end{aligned}
$$

Proof.

$$
\begin{aligned}
\mathrm{E} & \left(\operatorname{Tr}_{\gamma}\left(O^{\epsilon_{1}} Y_{1} \cdots O^{\epsilon_{n}} Y_{n}\right)\right) \\
& =\sum_{j_{1}, \ldots, j_{-n}=1}^{d} \mathrm{E}\left(o_{j_{1} j_{-1}}^{\left(\epsilon_{1}\right)} \cdots o_{j_{n} j_{-n}}^{\left(\epsilon_{n}\right)}\right) \mathrm{E}\left(y_{j_{-1} j_{\gamma(1)}}^{(1)} \cdots y_{j_{-n} j_{\gamma(n)}}^{(n)}\right)
\end{aligned}
$$

Now for notational convenience let $\epsilon(k)=\epsilon_{|k|} k$ and let $l_{k}=j_{\epsilon(k)}$, then $o_{j_{k} j_{-k}}^{\left(\epsilon_{k}\right)}=o_{l_{k} l_{-k}}$. Thus

$$
\mathrm{E}\left(o_{j_{1} j_{-1}}^{\left(\epsilon_{1}\right)} \cdots o_{j_{n} j_{-n}}^{\left(\epsilon_{n}\right)}\right)=\mathrm{E}\left(o_{l_{1} l_{-1}} \cdots o_{l_{n} l_{-n}}\right)=\sum_{p, q \in \mathcal{P}_{2}(n)}\langle\mathrm{Wg}(p), q\rangle \delta_{p \delta q \delta}^{l},
$$

where $\delta_{p \delta q \delta}^{l}=1$ if $l=l \circ p \delta q \delta$. Also $y_{j_{-k} j_{k+1}}^{(k)}=y_{l_{-\epsilon(k)} l_{\epsilon \gamma(k)}}^{(k)}$. Hence we have

$$
\begin{aligned}
\mathrm{E} & \left(\operatorname{Tr}_{\gamma}\left(O^{\epsilon_{1}} Y_{1} \cdots O^{\epsilon_{n}} Y_{n}\right)\right) \\
& =\sum_{l_{1}, \ldots, l_{-n}} \mathrm{E}\left(o_{l_{1} l_{-1}} \cdots o_{l_{n} l_{-n}}\right) \mathrm{E}\left(y_{l_{-\epsilon(1)} l_{\epsilon \gamma(1)}}^{(1)} \cdots y_{\left.l_{-\epsilon(n)} l_{\epsilon \gamma(n)}^{(n)}\right)}\langle\operatorname{Wg}(p), q\rangle \sum_{\substack{l_{1}, \ldots, l_{-n} \\
l=l o p \delta q \delta}} \mathrm{E}\left(y_{l_{-\epsilon(1)} l_{\epsilon \gamma(1)}}^{(1)} \cdots y_{l_{-\epsilon(n)} l_{\epsilon \gamma(n)}}^{(n)}\right) .\right.
\end{aligned}
$$

Let $i=l \circ \epsilon \gamma \delta$. Then $i_{1}=l_{-\epsilon(1)}, i_{-1}=l_{\epsilon \gamma(1)}, \ldots, i_{n}=l_{-\epsilon(n)}, i_{-n}=$ $l_{\epsilon \gamma(n)}$. Thus as $p \cdot{ }_{\epsilon} q=\delta \gamma^{-1} \delta_{\epsilon} p \delta q \delta \delta_{\epsilon} \gamma \delta$ we have

$$
\sum_{\substack{l_{1}, \ldots, l_{-n} \\ l=l o p \delta q \delta}} \mathrm{E}\left(y_{l_{-\epsilon(1)} l_{\epsilon \gamma(1)}}^{(1)} \cdots y_{l_{-\epsilon(n)} l_{\epsilon \gamma(n)}}^{(n)}\right)=\sum_{\substack{i_{1}, \ldots, i_{-n} \\ i=i \circ p \cdot{ }_{\epsilon} q}} \mathrm{E}\left(y_{i_{1} i_{-1}}^{(1)} \cdots y_{i_{n} i_{-n}}^{(n)}\right)
$$


So

$$
\begin{aligned}
\mathrm{E} & \left(\operatorname{Tr}_{\gamma}\left(O^{\epsilon_{1}} Y_{1} \cdots O^{\epsilon_{n}} Y_{n}\right)\right) \\
& =\sum_{p, q \in \mathcal{P}_{2}(n)}\langle\mathrm{Wg}(p), q\rangle \sum_{\substack{i_{1}, \ldots, i_{-n} \\
i=i \circ p \cdot \epsilon q}} \mathrm{E}\left(y_{i_{1} i_{-1}}^{(1)} \cdots y_{i_{n} i_{-n}}^{(n)}\right) \\
& =\sum_{p, q \in \mathcal{P}_{2}(n)}\langle\mathrm{Wg}(p), q\rangle \mathrm{E}\left(\operatorname{Tr}_{\left(\pi_{p \cdot \epsilon q}, \eta_{p \cdot \epsilon q}\right)}\left(Y_{1}, \ldots, Y_{n}\right)\right) .
\end{aligned}
$$

We shall need a special case of this result in section 9. Let us say that a permutation $\pi$ is parity preserving if for all $k, \pi(k)$ and $k$ have the same parity.

Lemma 13. Let $n_{1}, n_{2}, \ldots, n_{r}$ be even positive integers and $n=n_{1}+$ $\cdots+n_{r} . \quad$ Let $\gamma=\left(1,2, \ldots, n_{1}\right)\left(n_{1}+1, \ldots, n_{1}+n_{2}\right) \cdots\left(n_{1}+\cdots+\right.$ $\left.n_{r-1}+1, \ldots, n_{1}+\cdots+n_{r}\right) \in S_{n}$. Suppose that $\epsilon \in \mathbb{Z}_{2}^{n}$ is such that $\epsilon_{k}=(-1)^{k+1}$. Then for all $p, q \in \mathcal{P}_{2}(n), \pi_{p \cdot{ }_{\epsilon} q}$ is parity preserving.

Proof. We first show that $p \cdot{ }_{\epsilon} q=\delta \gamma^{-1} \delta_{\epsilon} p \delta q \delta \delta_{\epsilon} \gamma \delta$ is parity preserving. By direct computation we have the following.

$$
\begin{aligned}
p \cdot{ }_{\epsilon} q(2 k-1) & =\left\{\begin{array}{cc}
-\gamma^{-1}(q(2 k-1)) & q(2 k-1) \text { is even, } \\
q(2 k-1) & q(2 k-1) \text { is odd }
\end{array}\right. \\
p \cdot{ }_{\epsilon} q(-(2 k-1)) & =\left\{\begin{array}{cc}
-\gamma^{-1}(q(2 k)) & q(2 k) \text { is even, } \\
q(2 k) & q(2 k) \text { is odd } .
\end{array}\right.
\end{aligned}
$$

Note that since $\gamma$ always reverses the parity of its argument, all four possible outcomes are odd. Thus $p \cdot{ }_{\epsilon} q$ takes odd numbers to odd numbers. Since $p \cdot{ }_{\epsilon} q$ is a permutation it must then take even numbers to even numbers. Indeed

$$
\begin{aligned}
p \cdot{ }_{\epsilon} q(2 k) & =\left\{\begin{array}{cc}
p(2 k) & p(2 k) \text { is even } \\
-\gamma^{-1}(p(2 k)) & p(2 k) \text { is odd }
\end{array}\right. \\
p \cdot{ }_{\epsilon} q(-(2 k)) & =\left\{\begin{array}{cc}
p(\gamma(2 k)) & p(\gamma(2 k)) \text { is even } \\
-\gamma^{-1}(p(\gamma(2 k))) & p(\gamma(2 k)) \text { is odd }
\end{array}\right.
\end{aligned}
$$

Now $\delta(k)=-k$ is parity preserving, thus so is $\left(p \cdot{ }_{\epsilon} q\right) \delta$. Finally $\pi_{p \cdot{ }_{\epsilon} q}$ is obtained by choosing one representative of each pair $\left\{c, \delta c^{-1} \delta\right\}$ of $\left(p \cdot{ }_{\epsilon} q\right) \delta$, and taking the absolute value of each entry. This means that each cycle will consist of integers of the same parity. Hence $\pi_{p{ }_{\epsilon} q}$ is parity preserving.

We wish to extend the conclusion of Proposition 12 to case where some of the $Y$ 's are not interleaved by orthogonal matrices. 
Proposition 14. Let $O$ be a Haar distributed $d \times d$ random orthogonal matrix and $\left\{Y_{1}, \ldots, Y_{n}\right\} d \times d$ random matrices which are independent from $O$ and whose entries have moments of all orders. Let $1 \leq m \leq n$, $\gamma \in S_{m}, \epsilon \in \mathbb{Z}_{2}^{m}$ and suppose $d \geq m$.

$$
\begin{aligned}
& \mathrm{E}\left(\operatorname{Tr}_{\gamma}\left(O^{\epsilon_{1}} Y_{1}, \ldots, O^{\epsilon_{m}} Y_{m}\right) \operatorname{Tr}\left(Y_{m+1}\right) \cdots \operatorname{Tr}\left(Y_{n}\right)\right) \\
& \quad=\sum_{p, q \in \mathcal{P}_{2}(m)}\langle\mathrm{Wg}(p), q\rangle \mathrm{E}\left(\operatorname{Tr}_{\left(\pi_{p \cdot \epsilon q}, \eta_{p \cdot \epsilon q}\right)}\left(Y_{1}, \cdots, Y_{m}\right) \operatorname{Tr}\left(Y_{m+1}\right) \cdots \operatorname{Tr}\left(Y_{n}\right)\right)
\end{aligned}
$$

Proof. The proof is the same as for Proposition 12 except that we append the random variable $\operatorname{Tr}\left(Y_{m+1}\right) \cdots \operatorname{Tr}\left(Y_{n}\right)$ to the right hand side of each expression.

We now wish to extend the conclusion of Proposition 12 in another way, namely to the case of independent Haar distributed orthogonal matrices. Suppose $\left\{O_{1}, \ldots, O_{s}\right\}$ are independent Haar distributed $d \times d$ orthogonal matrices, with the $(i, j)$ entry of $O_{k}$ denoted $o_{(k) i j}$. We shall

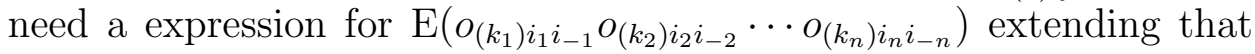
given in Theorem 9 .

Notation 15. Given an $n$-tuple $\left(i_{1}, i_{2}, \ldots, i_{n}\right)$ of integers in $[s]$ we let $\operatorname{ker}(i)$ be the partition of $[n]$ such that $i_{r}=i_{s}$ where $r$ and $s$ are in the same block of $\operatorname{ker}(i)$ and $i_{r} \neq i_{s}$ when $r$ and $s$ are in different blocks of $\operatorname{ker}(i)$.

Let $\mathcal{U} \in \mathcal{P}(n)$ be a partition of $[n]$ and $p \in \mathcal{P}_{2}(n)$ be a pairing such that each pair of $p$ lies in some block of $\mathcal{U}$. We shall denote this by $p \leq \mathcal{U}$. If we write the blocks of $\mathcal{U}$ as $\left\{U_{1}, \ldots, U_{r}\right\}$, then the pairs of $p$ that lie in $U_{i}$ form a pairing of $U_{i}$ which we shall denote by $p_{\mid U_{i}}$ or just $p_{i}$ when convenient.

If we have a partition $\mathcal{U}$ and pairings $p, q \in \mathcal{P}_{2}(n)$ with $p, q \leq \mathcal{U}$ then we let

$$
\mathrm{Wg}(\mathcal{U}, p, q)=\left\langle\mathrm{Wg}\left(p_{1}\right), q_{1}\right\rangle \cdots\left\langle\mathrm{Wg}\left(p_{r}\right), q_{r}\right\rangle
$$

Remark 16. Note that since $\mathrm{Wg}$ is not multiplicative, $\operatorname{Wg}(\mathcal{U}, p, q)$ and $\langle\mathrm{Wg}(p), q\rangle$ are different. However by Remark 8 we see that when $p, q \leq$ $\mathcal{U}$ then $\operatorname{Wg}(\mathcal{U}, p, q)-\langle\operatorname{Wg}(p), q\rangle=\mathrm{O}\left(d^{-n+\#(p \vee q)-1}\right)$ as the leading terms in both expressions are the same.

Lemma 17. Suppose $\left\{O_{1}, \ldots, O_{s}\right\}$ are independent Haar distributed $d \times d$ orthogonal matrices. Let the $(i, j)$ entry of $O_{k}$ be denoted $o_{(k) i, j}$. Given an $n$-tuple $\left(k_{1}, \ldots, k_{n}\right)$ in $[s]$ then

$$
\mathrm{E}\left(o_{\left(k_{1}\right) i_{1} i_{-1}} o_{\left(k_{2}\right) i_{2} i_{-2}} \cdots o_{\left(k_{n}\right) i_{n} i_{-n}}\right)=\sum_{\substack{p, q \in \mathcal{P}_{2}(n) \\ p, q \leq \operatorname{ker}(k)}} \operatorname{Wg}(\operatorname{ker}(k), p, q) \delta_{p \delta q \delta}^{i} .
$$


Proof. We can write $\mathrm{E}\left(o_{\left(k_{1}\right) i_{1} i_{-1}} o_{\left(k_{2}\right) i_{2} i_{-2}} \cdots o_{\left(k_{n}\right) i_{n} i_{-n}}\right)$ as a product of expectations, one for each block of $\operatorname{ker}(k)$. For each block $U_{j}$ of $\operatorname{ker}(k)$ we get a factor $\sum_{p_{j}, q_{j} \in \mathcal{P}_{2}\left(U_{i}\right)}\left\langle\mathrm{Wg}\left(p_{j}\right), q_{j}\right\rangle d_{p_{j} \delta q_{j} \delta}^{i_{j} \delta}$ where $i_{j}$ is the restriction of $i$ to the block $U_{j}$. Taking the product of these terms we get $\sum_{\substack{p, q \in \mathcal{P}_{2}(n) \\ p, q \leq \operatorname{ker}(k)}} \mathrm{Wg}(\operatorname{ker}(k), p, q) \delta_{p \delta q \delta}^{i}$.

Proposition 18. Let $\left\{O_{1}, \ldots, O_{s}\right\}$ be independent Haar distributed $d \times d$ orthogonal matrices and $\left\{Y_{1}, \ldots, Y_{n}\right\} d \times d$ random matrices which are independent from $\left\{O_{1}, \ldots, O_{s}\right\}$ and whose entries have moments of all orders. Let $\gamma \in S_{n}, \epsilon \in \mathbb{Z}_{2}^{n}$ and suppose $d \geq n$. For each $n$-tuple $\left(k_{1}, \ldots, k_{n}\right)$ in $[s]$ we have

$$
\begin{aligned}
& \mathrm{E}\left(\operatorname{Tr}_{\gamma}\left(O_{k_{1}}^{\epsilon_{1}} Y_{1}, \ldots, O_{k_{n}}^{\epsilon_{n}} Y_{n}\right)\right) \\
& =\sum_{\substack{p, q \in \mathcal{P}_{2}(n) \\
p, q \leq \operatorname{ker}(k)}} \operatorname{Wg}(\operatorname{ker}(k), p, q) \mathrm{E}\left(\operatorname{Tr}_{\left(\pi_{p \cdot \epsilon^{q}, \eta_{p} \epsilon_{q}}\right)}\left(Y_{1}, \cdots, Y_{n}\right)\right) .
\end{aligned}
$$

Proof. The only point where the proof differs from the proof of Proposition 12 is in Equation 5, which we replace by

$$
\begin{aligned}
& \mathrm{E}\left(o_{\left(k_{1}\right) j_{1} j_{-1}}^{\left(\epsilon_{1}\right)} \cdots o_{\left(k_{n}\right) j_{n} j_{-n}}^{\left(\epsilon_{n}\right)}\right) \\
& \quad=\mathrm{E}\left(o_{\left(k_{1}\right) l_{1} l_{-1}} \cdots o_{\left(k_{n}\right) l_{n} l_{-n}}\right)=\sum_{\substack{p, q \in \mathcal{P}_{2}(n) \\
p, q \leq \operatorname{ker}(k)}} \mathrm{Wg}(\operatorname{ker}(k), p, q) \delta_{p \delta q \delta}^{l} .
\end{aligned}
$$

The remainder of the proof is unchanged.

\section{A Lemma on Spoke Diagrams}

At several points later on we shall wish to know that a given permutation represents a spoke diagram (see Figure 2). Lemma 20 identifies standard spoke diagrams and Lemma 21 identifies reversed spoke diagrams.

Lemma 19. Suppose $\gamma \in S_{n}$ is a permutation, $p \in \mathcal{P}_{2}(n)$ a pairing, and $\epsilon \in \mathbb{Z}_{2}^{n}$ an assignment of signs, are such that $\pi_{p_{\epsilon} p}$ is a pairing. Let $(r, s) \in p$ be a pair of $p$.

i) If $\epsilon_{r}=-\epsilon_{s}$ then $\left(\gamma^{-1}(r), \gamma(s)\right) \in p,\left(\gamma^{-1}(r), s\right) \in \pi_{p \cdot \epsilon p}$, and $\epsilon_{\gamma^{-1}(r)}=-\epsilon_{\gamma(s)}$.

ii) If $\epsilon_{r}=\epsilon_{s}$ then $\left(\gamma^{-1}(r), \gamma^{-1}(s)\right) \in p,\left(\gamma^{-1}(r), \gamma^{-1}(s)\right) \in \pi_{p \cdot \epsilon p}$, and $\epsilon_{\gamma(r)}=\epsilon_{\gamma(s)}$. 
Proof. (i) Let us suppose that $\epsilon_{r}=-\epsilon_{s}$. Since $(r, s) \in p$ and $\epsilon_{r}=-\epsilon_{s}$ we have

$$
(r,-s),(-r, s) \in \delta_{\epsilon} p \delta p \delta \delta_{\epsilon} .
$$

Since $\pi_{p \cdot \epsilon} p$ is a pairing, $\left(p \cdot{ }_{\epsilon} p\right) \delta$ is also a pairing - recall that $p \cdot{ }_{\epsilon} p=$ $(\gamma \delta)^{-1} \delta_{\epsilon} p \delta p \delta \delta_{\epsilon}(\gamma \delta)$. Also

$$
\left(p \cdot{ }_{\epsilon} p\right) \delta\left(\gamma^{-1}(r)\right)=(\gamma \delta)^{-1}\left(\delta_{\epsilon} p \delta p \delta \delta_{\epsilon}\right)(\gamma \delta) \delta\left(\gamma^{-1}(r)\right)=s .
$$

Thus $\left(\gamma^{-1}(r), s\right) \in\left(p \cdot{ }_{\epsilon} p\right) \delta$, because $\left(p \cdot{ }_{\epsilon} p\right) \delta$ is a pairing. Since both $\gamma^{-1}(r), s \in[n]$ we have that $\left(\gamma^{-1}(r), s\right) \in \pi_{p \cdot \epsilon p}$. Moreover $\left(\gamma^{-1}(r)\right.$, $s) \in \pi_{p \cdot{ }_{\epsilon} p}$ and so $\left(p \cdot{ }_{\epsilon} p\right) \delta(s)=\gamma^{-1}(r)$. Unwinding this equation we have

$$
p \delta p \delta\left(\epsilon_{\gamma(s)} \gamma(s)\right)=-\epsilon_{\gamma^{-1}(r)} \gamma^{-1}(r) .
$$

Since $p \delta p \delta$, as a permutation, doesn't change the sign of its argument, we have $\epsilon_{\gamma(s)}=-\epsilon_{\gamma^{-1}(r)}$. Thus $p \delta p \delta(\gamma(s))=\gamma^{-1}(r)$, and we are left with $\left(\gamma^{-1}(r), \gamma(s)\right)$ is a cycle of $p,\left(\gamma^{-1}(r), s\right) \in \pi_{p \cdot \epsilon}$, and $\epsilon_{\gamma(s)}=-\epsilon_{\gamma^{-1}(r)}$ as required.

(ii) Let us suppose that $\epsilon_{r}=\epsilon_{s}$. Since $(r, s) \in p$ and $\epsilon_{r}=\epsilon_{s}$ we have

$$
(r, s),(-r,-s) \in \delta_{\epsilon} p \delta p \delta \delta_{\epsilon} .
$$

Since $\pi_{p \cdot \epsilon p}$ is also a pairing, $\left(p \cdot{ }_{\epsilon} p\right) \delta$ is a pairing. Also

$$
\left(p \cdot{ }_{\epsilon} p\right) \delta\left(\gamma^{-1}(r)\right)=(\gamma \delta)^{-1}\left(\delta_{\epsilon} p \delta p \delta \delta_{\epsilon}\right)(\gamma \delta) \delta\left(\gamma^{-1}(r)\right)=-\gamma^{-1}(s) .
$$

Thus $\left(\gamma^{-1}(r),-\gamma^{-1}(s)\right)$ is a pair of $\left(p \cdot{ }_{\epsilon} p\right) \delta$. Thus $\left(\gamma^{-1}(r), \gamma^{-1}(s)\right) \in$ $\pi_{p \cdot \epsilon p}$. Moreover $\left(p \cdot_{\epsilon} p\right) \delta\left(-\gamma^{-1}(s)\right)=\gamma^{-1}(r)$. Unwinding the equation $\left(p \cdot{ }_{\epsilon} p\right) \delta\left(-\gamma^{-1}(s)\right)=\gamma^{-1}(r)$ we have

$$
p \delta p \delta\left(-\epsilon_{\gamma(s)} \gamma(s)\right)=-\epsilon_{\gamma^{-1}(r)} \gamma^{-1}(r) .
$$

Since $p \delta p \delta$, as a permutation, doesn't change the sign of its argument, we have $\epsilon_{\gamma^{-1}(r)}=\epsilon_{\gamma^{-1}(s)}$. Thus $p \delta p \delta\left(\gamma^{-1}(s)\right)=\gamma^{-1}(r)$, and we are left with $\left(\gamma^{-1}(r), \gamma^{-1}(s)\right)$ is a cycle of $p, \epsilon_{\gamma^{-1}(r)}=\epsilon_{\gamma^{-1}(s)}$, and $\left(\gamma^{-1}(r), \gamma^{-1}(s)\right) \in \pi_{p \cdot \epsilon p}$ as claimed.

Lemma 20. Let $\gamma$ be the permutation with the two cycles $(1, \ldots, m)$ $(m+1, \ldots, m+n)$, let $\epsilon \in \mathbb{Z}_{2}^{m+n}$, and let $p \in \mathcal{P}_{2}(m+n)$ be a pairing such that

i) $p \vee \gamma=1_{m+n}$, i.e. at least one of cycle of $p$ connects the two cycles of $\gamma$;

ii) for some $(r, s) \in p$ we have $\epsilon_{r}=-\epsilon_{s}$; and

iii) $\pi_{p \cdot{ }_{\epsilon} p}$ is a pairing. 
Then $m=n, p$ and $\pi_{p \cdot{ }_{\epsilon} p}$ are standard spoke diagrams, and there is $l$, which we make take to be $\gamma^{-r}(s)$ if we assume that $1 \leq r \leq m$, such that

a) every cycle of $p$ is of the form $\left(k, \gamma^{-k}(l)\right)$ for $1 \leq k \leq m$, and

b) every cycle of $\pi_{p \cdot{ }_{\epsilon} p}$ is of the form $\left(k, \gamma^{-k-1}(l)\right)$ for $1 \leq k \leq m$,

c) and $\epsilon_{r}=-\epsilon_{s}$ for all $(r, s) \in p$,

d) $\eta_{k}=1$ for all $k$.

Proof. Let $(r, s) \in p$, i.e. $(r, s)$ is a cycle of $p$, and suppose $\epsilon_{r}=-\epsilon_{s}$. By using induction on Lemma 19 we know that for all $k,\left(\gamma^{-k}(r), \gamma^{k}(s)\right) \in$ $p, \epsilon_{\gamma^{-k}(r)}=-\epsilon_{\gamma^{k}(s)}$, and $\left(\gamma^{-k}(r), \gamma^{k-1}(s)\right) \in \pi_{p \cdot \epsilon p}$. Recall that in the proof of Lemma $19(i)$ we showed that $\left(\gamma^{-1}(r), s\right) \in\left(p \cdot{ }_{\epsilon} p\right) \delta$. This implied that $\left(\gamma^{-1}(r), s\right) \in \pi_{p \cdot \epsilon_{\epsilon} p}$ and that $\eta_{\gamma^{-1}(r)}=\eta_{s}=1$. By our induction argument we have that $\eta_{k}=1$ for all $k$.

By assumption, $p$ has at least one pair $(r, s)$ that connects the cycles of $\gamma$; and so by what we have just observed, all cycles of $p$ connect the two cycles of $\gamma$. This implies $m=n$, and all cycles of $p$ are of the form $\left(k, \gamma^{-k}(l)\right)$, where $l=\gamma^{r-1}(s)$, assuming $\gamma^{-r}(r)=m$. Moreover, both $p$ and $\pi_{p \cdot{ }_{\epsilon} p}$ are spoke diagrams, i.e. non-crossing annular pairings of an $(m, m)$-annulus with all pairs connecting the two circles; see Figure 2.

Lemma 21. Let $\gamma$ be the permutation with the two cycles $(1, \ldots, m)$ $(m+1, \ldots, m+n)$, let $\epsilon \in \mathbb{Z}_{2}^{m+n}$, and let $p \in \mathcal{P}_{2}(m+n)$ be a pairing such that

i) $p \vee \gamma=1_{m+n}$, i.e. at least one of cycle of $p$ connects the two cycles of $\gamma$

ii) for some $(r, s) \in p$ we have $\epsilon_{r}=\epsilon_{s}$; and

iii) $\pi_{p \cdot{ }_{\epsilon} p}$ is a pairing.

Then $m=n, p$ and $\pi_{p \cdot{ }_{\epsilon} p}$ are reversed spoke diagrams, and there is $l$, which we make take to be $\gamma^{-r}(s)$ if we assume that $1 \leq r \leq m$, such that

a) every cycle of $p$ is of the form $\left(k, \gamma^{k}(l)\right)$ for $1 \leq k \leq m$, and

$b)$ every cycle of $\pi_{p \cdot{ }_{\epsilon} p}$ is of the form $\left(k, \gamma^{k}(l)\right)$ for $1 \leq k \leq m$,

c) and $\epsilon_{r}=\epsilon_{s}$ for all $(r, s) \in p$,

d) $\eta_{k}=-1$ for all $k \in[m]$.

Proof. Let $(r, s) \in p$, i.e. $(r, s)$ is a cycle of $p$, and suppose $\epsilon_{r}=\epsilon_{s}$. By using induction on Lemma 19 we know that for all $k,\left(\gamma^{-k}(r), \gamma^{-k}(s)\right) \in$ $p, \epsilon_{\gamma^{-k}(r)}=\epsilon_{\gamma^{-k}(s)}$, and $\left(\gamma^{-k}(r), \gamma^{-k}(s)\right) \in \pi_{p \cdot{ }_{\epsilon} p}$. Recall that in the proof of Lemma 19 (ii) we showed that $\left(\gamma^{-1}(r),-\gamma^{-1}(s)\right) \in\left(p \cdot{ }_{\epsilon} p\right) \delta$. This implied that $\left(\gamma^{-1}(r), \gamma^{-1}(s)\right) \in \pi_{p \cdot{ }_{\epsilon} p}$ and that $\eta_{\gamma^{-1}(r)}=-1$. By our induction argument we have that $\eta_{k}=-1$ for all $k \in[m]$. 
By assumption, $p$ has at least one pair $(r, s)$ that connects the cycles of $\gamma$; and so by what we have just observed, all cycles of $p$ connect the two cycles of $\gamma$. This implies $m=n$, and all cycles of $p$ are of the form $\left(k, \gamma^{k}(l)\right)$, where $l=\gamma^{-r}(s)$, assuming $\gamma^{-r}(r)=m$. Moreover, both $p$ and $\pi_{p \cdot{ }_{\epsilon} p}$ are spoke diagrams, i.e. non-crossing annular pairings of an $(m, m)$-annulus with all pairs connecting the two circles; see Figure 2.

Corollary 22. Let $\gamma \in S_{n}$ be a permutation, $p \in \mathcal{P}_{2}(n)$ a pairing, and $\epsilon \in \mathbb{Z}_{2}^{n}$ an assignment of signs. Suppose that $\pi_{p \cdot{ }_{\epsilon} p}$ is a pairing then each block of $p \vee \gamma$ contains at most two cycles of $\gamma$.

Proof. We saw in Lemma 19 that when $p$ connects a pair of cycles of $\gamma$ these two cycles form a spoke diagram. So a block of $p \vee \gamma$ can contain at most two cycles of $\gamma$.

\section{Real Second Order Freeness}

Let us recall the definition of real second order freeness from Redelmeier $\left[\mathrm{R}_{2}, \S 1\right]$. We begin with the concept of a real second order non-commutative probability space.

Definition 23. Let $\mathcal{A}$ be an algebra over $\mathbb{C}$ and with an anti-automorphism of order 2 denoted by $a \mapsto a^{t}$. Suppose that $\varphi: \mathcal{A} \rightarrow \mathbb{C}$ is a tracial state and $\varphi_{2}: \mathcal{A} \times \mathcal{A} \rightarrow \mathbb{C}$ is a bi-trace, i.e. $\varphi_{2}$ is bilinear and tracial in each entry. Moreover we assume that $\varphi_{2}(1, a)=\varphi(a, 1)=0$, $\varphi\left(a^{t}\right)=\varphi(a)$ and $\varphi_{2}\left(a^{t}, b\right)=\varphi_{2}\left(a, b^{t}\right)=\varphi_{2}(a, b)$ for all $a, b \in \mathcal{A}$. Then $\left(\mathcal{A}, \varphi, \varphi_{2}, t\right)$ is a real second order non-commutative probability space.

Notation 24. Let unital subalgebras $\mathcal{A}_{1}, \ldots, \mathcal{A}_{r} \subset \mathcal{A}$ be given.

$i)$ We say that a tuple $\left(a_{1}, \ldots, a_{n}\right)$ of elements from $\mathcal{A}$ is cyclically alternating if, for each $i$, there is $j_{i} \in\{1, \ldots, r\}$ such that $a_{i} \in \mathcal{A}_{j_{i}}$ and, if $n \geq 2$, we have $j_{k} \neq j_{k+1}$ for all $k=1, \ldots, n$. We count indices in a cyclic way modulo $n$, i.e., for $k=n$ the equation above means $j_{n} \neq j_{1}$.

ii) We say that a tuple $\left(a_{1}, \ldots, a_{n}\right)$ of elements from $\mathcal{A}$ is centred if we have

$$
\varphi\left(a_{i}\right)=0 \quad \text { for all } i=1, \ldots, n .
$$

Definition 25. Let $\left(\mathcal{A}, \varphi, \varphi_{2}, t\right)$ be a real second order non-commutative probability space and suppose that we have unital subalgebras $\mathcal{A}_{1}, \ldots, \mathcal{A}_{n}$ that are invariant under $a \mapsto a^{t}$. We say that $\mathcal{A}_{1}, \ldots, \mathcal{A}_{n}$ are real free of second order if (see figure 4 )

i) the subalgebras $\mathcal{A}_{1}, \ldots, \mathcal{A}_{n}$ are free with respect to $\varphi$; 

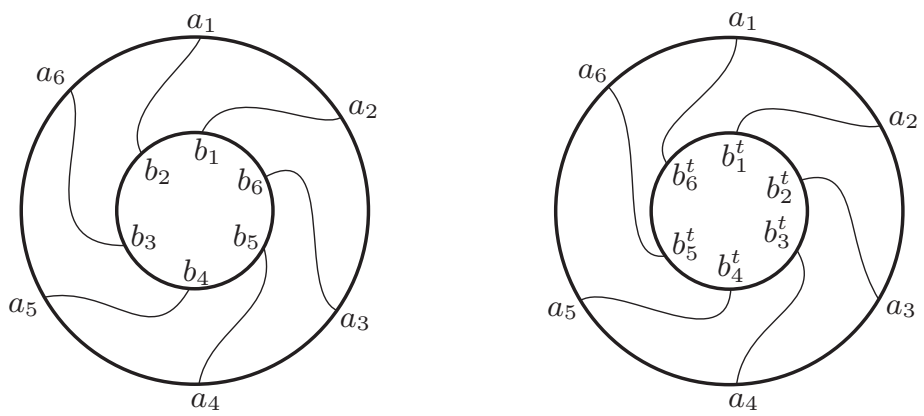

FIGURE 4. The terms on the right hand side of equation (6) are sums over all spoke diagrams. In the diagram on the left the circles have the opposite orientation; we put the $a$ 's on on circle and the $b$ 's on the other. This gives the first term on the right hand side of (6). In the circle on the right the two circles have the same orientation and we put ' $b^{t}$ 's on the inside circle. This gives the second term on the right hand side of (6).

ii) for every $a_{1}, \ldots, a_{m} \in \mathcal{A}$ and $b_{1}, \ldots, b_{n} \in \mathcal{A}$ such that $\left(a_{1}, \ldots\right.$, $\left.a_{m}\right)$ and $\left(b_{1}, \ldots, b_{n}\right)$ are centred and cyclically alternating, we have

a) $\varphi_{2}\left(a_{1} \cdots a_{m}, b_{1} \cdots b_{n}\right)=0$, if $m \neq n$ or if $m=n=1$ and $a_{1}$ and $b_{1}$ are from different subalgebras;

b) for $m=n>1$ we have, taking all indices modulo $n$

$$
\varphi_{2}\left(a_{1} \cdots a_{n}, b_{1} \cdots b_{n}\right)=\sum_{k=1}^{n} \prod_{i=1}^{n}\left(\varphi\left(a_{i} b_{k-i}\right)+\varphi\left(a_{i} b_{i-k}^{t}\right)\right) .
$$

Notation 26. Let $p \in \mathbb{C}\left[x_{1}, \ldots, x_{s}, x_{1}^{t}, \ldots, x_{s}^{t}\right]$ be a polynomial in the non-commuting variables $\left\{x_{1}, \ldots, x_{s}, x_{1}^{t}, \ldots, x_{s}^{t}\right\}$ and $A_{1}, \ldots, A_{s}$ be $d \times$ $d$ matrices. By $p\left(A_{1}, \ldots, A_{s}\right)$ we mean the matrix obtained by replacing $x_{i}$ by $A_{i}$ and $x_{i}^{t}$ by $A_{i}^{t}$ in $p$. Similarly if $\left(\mathcal{A}, \varphi, \varphi_{2}, t\right)$ is a real second order non-commutative probability space then by $p\left(a_{1}, \ldots, a_{s}\right)$ we mean the random variable in $\mathcal{A}$ obtained by replacing $x_{i}$ by $a_{i}$ and $x_{i}^{t}$ by $a_{i}^{t}$.

Remark 27. Expanding on the notation in equation (3) we define, for a permutation $\pi \in S_{n}$ and $a_{1}, \ldots, a_{n} \in \mathcal{A}, \varphi_{\pi}\left(A_{1}, \ldots, a_{n}\right)$ as below.

$$
\varphi_{\pi}\left(a_{1}, \ldots, a_{n}\right)=\prod_{\substack{c \in \pi \\ c=\left(i_{1}, \ldots, i_{k}\right)}} \varphi\left(a_{i_{1}} \cdots a_{i_{k}}\right),
$$


where the product is over all cycles $c$ of $\pi$ and for each cycle $c=$ $\left(i_{1}, \ldots, i_{k}\right)$ we get the factor $\varphi\left(a_{i_{1}}, \ldots, a_{i_{k}}\right)$. This makes $\varphi_{\pi}$ a $n$-linear functional.

With this notation we can write equation (6) in a simpler way:

$$
\begin{aligned}
& \varphi_{2}\left(a_{1} \cdots a_{n}, b_{1} \cdots b_{n}\right) \\
& =\sum_{\pi \in S p^{+}(n)} \varphi_{\pi}\left(a_{1}, \ldots, a_{n}, b_{1}, \ldots, b_{n}\right) \\
& +\sum_{\pi \in S p^{-}(n)} \varphi_{\pi}\left(a_{1}, \ldots, a_{n}, b_{1}^{t}, \ldots, b_{n}^{t}\right),
\end{aligned}
$$

where, recall, $S p^{+}(n)$ denotes the set of standard spoke diagrams and $S p^{-}(n)$ denotes the set of standard spoke diagrams.

We shall need to use the associativity of real second order freeness. Let us recall how this works in the first order case [VDN]. Suppose that we have unital subalgebras $\mathcal{A}_{1}, \ldots, \mathcal{A}_{s} \subset \mathcal{A}$ which are free with respect to $\varphi$. Moreover that for each $1 \leq i \leq s$ we have unital subalgebras $\mathcal{B}_{i, 1}, \ldots, \mathcal{B}_{i, t_{i}} \subset \mathcal{A}_{i}$ which are free with respect to $\varphi$. Then by [VDN, Prop. 2.5.5 (iii)] the subalgebras $\mathcal{B}_{1,1}, \ldots \mathcal{B}_{s, t_{s}} \subset \mathcal{A}$ are free with respect to $\varphi$. We shall prove the real second order version of this. In [MŚS, Remark 2.7] the second order version of [VDN] was left as an exercise for the reader, now we shall provide a solution. We begin with a lemma.

Lemma 28. Let $\mathcal{A}_{1}, \ldots, \mathcal{A}_{s} \subset \mathcal{A}$ be unital subalgebras which are free with respect to $\varphi$. Suppose that $a_{1}, \ldots, a_{m}, b_{1}, \ldots, b_{n} \in \mathcal{A}$ are such that

$\circ \varphi\left(a_{i}\right)=\varphi\left(b_{j}\right)=0$ for all $i$ and $j$;

$\circ a_{i} \in \mathcal{A}_{k_{i}}$ and $k_{1} \neq k_{2} \neq \cdots \neq k_{m}$

$\circ b_{j} \in \mathcal{A}_{l_{j}}$ and $l_{1} \neq l_{2} \neq \cdots \neq l_{n}$.

Then for $m \neq n, \varphi\left(a_{1} \cdots a_{m} b_{n} \cdots b_{1}\right)=0$ and for $m=n$

$$
\varphi\left(a_{1} \cdots a_{m} b_{m} \cdots b_{1}\right)=\prod_{i=1}^{m} \varphi\left(a_{i} b_{i}\right) .
$$

Proof. Let us begin by showing that

$$
\varphi\left(a_{1} \cdots a_{m} b_{n} \cdots b_{1}\right)=\varphi\left(a_{m} b_{n}\right) \varphi\left(a_{1} \cdots a_{m-1} b_{n-1} \cdots b_{1}\right) .
$$

First suppose that $k_{m} \neq l_{n}$. Then both $\varphi\left(a_{1} \cdots a_{m} b_{n} \cdots b_{1}\right)$ and $\varphi\left(a_{m} b_{n}\right)$ are 0 by freeness. Thus both sides of the equation above are 0 . Next suppose that $k_{m}=l_{n}$ and write $a_{m} b_{n}=\left(a_{m} b_{n}\right)^{\circ}+\varphi\left(a_{m} b_{n}\right)$. Then $\varphi\left(a_{1} \cdots a_{m-1}\left(a_{m} b_{n}\right)^{\circ} b_{n-1} \cdots b_{1}\right)=0$ because $k_{m-1} \neq k_{m}=l_{n} \neq l_{n-1}$. Thus

$$
\varphi\left(a_{1} \cdots a_{n} b_{1} \cdots b_{1}\right)
$$




$$
\begin{aligned}
& =\varphi\left(a_{1} \cdots a_{m-1}\left(a_{m} b_{n}\right)^{\circ} b_{n-1} \cdots b_{1}\right) \\
& +\varphi\left(a_{m} b_{n}\right) \varphi\left(a_{1} \cdots a_{m-1} b_{n-1} \cdots b_{1}\right) \\
& =\varphi\left(a_{m} b_{n}\right) \varphi\left(a_{1} \cdots a_{m-1} b_{n-1} \cdots b_{1}\right) .
\end{aligned}
$$

Now we conclude by induction. If $m=n$ we get the formula we claimed. If $m<n$ then

$$
\varphi\left(a_{1} \cdots a_{m} b_{n} \cdots b_{1}\right)=\varphi\left(a_{m} b_{n}\right) \cdots \varphi\left(a_{m} b_{n-m+1}\right) \varphi\left(b_{n-m} \cdots b_{1}\right)=0
$$

by the freeness of the $b_{j}$ 's. The case when $m>n$ is exactly the same.

Proposition 29. Let $\mathcal{A}_{1}, \ldots, \mathcal{A}_{s} \subset \mathcal{A}$ be t-invariant unital subalgebras of $\mathcal{A}$ which are real second order free with respect to $\left(\varphi, \varphi_{2}\right)$. For each $1 \leq i \leq s$ suppose we have $t$-invariant unital subalgebras $\mathcal{B}_{i, 1} \ldots, \mathcal{B}_{i, t_{i}} \subset$ $\mathcal{A}_{i}$ which are real free of second order with respect to $\left(\varphi, \varphi_{2}\right)$. Then the subalgebras $\mathcal{B}_{1,1}, \ldots, \mathcal{B}_{S, t_{s}} \subset \mathcal{A}$ are real free of second order with respect to $\left(\varphi, \varphi_{2}\right)$.

Proof. The proof of first order freeness is as in [VDN, Prop. 2.5.5 (iii)]. So let us prove part (ii) of Definition 25. Let $a_{1}, \ldots, a_{m}, b_{1}, \ldots, b_{n} \in \mathcal{A}$ be such that

$$
\begin{aligned}
& \circ \varphi\left(a_{i}\right)=\varphi\left(b_{j}\right)=0 \text { for all } i \text { and } j \text {; and } \\
& \circ a_{i} \in \mathcal{B}_{k_{i}, u_{i}} \text { and }\left(k_{1}, u_{1}\right) \neq\left(k_{2}, u_{2}\right) \neq \cdots \neq\left(k_{m}, u_{m}\right) \neq\left(k_{1}, u_{1}\right) ; \\
& \quad \text { and } \\
& \circ b_{j} \in \mathcal{B}_{l_{j}, v_{j}} \text { and }\left(l_{1}, v_{1}\right) \neq\left(l_{2}, v_{2}\right) \neq \cdots \neq\left(l_{n}, v_{n}\right) \neq\left(l_{1}, v_{1}\right) .
\end{aligned}
$$

We must show that for $m=n \geq 2$

$$
\begin{aligned}
& \varphi_{2}\left(a_{1} \cdots a_{m}, b_{1} \cdots b_{m}\right) \\
&=\sum_{\pi \in S p^{+}(m)} \varphi_{\pi}\left(a_{1} \cdots a_{m}, b_{1} \cdots b_{m}\right) \\
&+\sum_{\pi \in S p^{-}(m)} \varphi_{\pi}\left(a_{1} \cdots a_{m}, b_{1}^{t} \cdots b_{m}^{t}\right)
\end{aligned}
$$

and is 0 for $m \neq n$; the case $m=n=1$ is immediate.

Note that adjacent $a_{i}$ 's are, by assumption, from different $\mathcal{B}_{k, v}$ 's but might be from the same $\mathcal{A}_{d}$. So we group the $a_{i}$ 's according to which $\mathcal{A}_{d}$ contains them. Let $m_{1}, \ldots, m_{p}$ be positive integers such that $m_{1}+\cdots+m_{p}=m$ and $a_{m_{1}+\cdots+m_{i-1}+1}, \ldots, a_{m_{1}+\cdots+m_{i}} \in \mathcal{A}_{d_{i}}$, for $1 \leq i \leq p$ and $d_{1} \neq d_{2} \neq \cdots \neq d_{p} \neq d_{1}$. Then we let $A_{i}=$ $a_{m_{1}+\cdots+m_{i-1}+1} \cdots a_{m_{1}+\cdots+m_{i}} \in \mathcal{A}_{d_{i}}$. Then $a_{1} \cdots a_{m}=A_{1} \cdots A_{p}$.

We do exactly the same for the $b_{j}$ 's. Namely we let $n_{1}, \ldots, n_{q}$ be positive integers such that $n_{1}+\cdots+n_{q}=n$ and $b_{n_{1}+\cdots n_{j-1}+1}, \ldots, b_{n_{1}+\cdots+n_{j}} \in$ 
$\mathcal{A}_{e_{j}}$ for $1 \leq j \leq q$ and $e_{1} \neq e_{2} \neq \cdots \neq e_{q} \neq e_{1}$. We let $B_{j}=$ $b_{n+1+\cdots+b_{j-1}+1} \cdots b_{n_{1}+\cdots n_{j}} \in \mathcal{A}_{e_{i}}$. Then $b_{1} \cdots b_{n}=B_{1} \cdots B_{q}$.

Note that by first order freeness

$$
\varphi\left(A_{i}\right)=\varphi\left(a_{m_{1}+\cdots+m_{i-1}+1} \cdots a_{m_{1}+\cdots+m_{i}}\right)=0,
$$

since the $\mathcal{B}_{i, j}$ 's are first order free by [VDN, Prop. 2.5.5 (iii)]. Likewise $\varphi\left(B_{j}\right)=0$.

If $p=q=1$ then we have (7) by the assumed second order freeness of $\mathcal{B}_{1,1}, \ldots, \mathcal{B}_{1, t_{1}}$. If $p \neq q$, then by the assumed second order freeness of $\mathcal{A}_{1}, \ldots, \mathcal{A}_{s}$ we have $\varphi_{2}\left(a_{1} \cdots a_{m}, b_{1} \cdots b_{n}\right)=0$, thus the left hand side of $(7)$ is 0 .

Let us consider the right hand side of (7). If $m \neq n$ then the right hand side is 0 . So let us suppose that $m=n$. Let us first consider the term involving $S p^{+}(m)$. For $\pi \in S p^{+}(m)$ and $\varphi_{\pi}\left(a_{1}, \ldots, a_{m}, b_{1}, \ldots, b_{n}\right)$ $\neq 0$ we must have $\left(k_{i}, i_{i}\right)=\left(l_{j}, v_{j}\right)$ for all $(i, j) \in \pi$. This means $\pi$ gives a bijection between the $\mathcal{A}_{i}$ 's which contain the $a_{i}$ 's and the $\mathcal{A}_{j}$ 's which contain the $b_{j}$ 's. So in particular $p=q$, which is impossible. Likewise if $\pi \in S p^{-}(m)$ then we have a bijection between the $\mathcal{A}_{i}$ 's containing the $a_{i}$ 's and the $\mathcal{A}_{j}$ 's containing the $b_{j}^{t}$ 's. So again we would have $p=q$.

Now let us suppose that $p=q \geq 2$. By the assumed real second order freeness of $\mathcal{A}_{1}, \ldots, \mathcal{A}_{s}$ we have

$$
\begin{aligned}
\varphi\left(a_{1} \cdots a_{m}, b_{1} \cdots b_{n}\right)=\varphi_{2}\left(A_{1} \cdots A_{p}, B_{1} \cdots B_{p}\right) \\
=\sum_{\pi \in S p^{+}(p)} \varphi_{\pi}\left(A_{1}, \ldots, A_{p}, B_{1}, \ldots, B_{p}\right) \\
+\sum_{\pi \in S p^{-}(p)} \varphi_{\pi}\left(A_{1}, \ldots, A_{p}, B_{1}^{t}, \ldots, B_{p}^{t}\right) .
\end{aligned}
$$

For $\pi \in S p^{+}(p)$ and $(i, j) \in \pi$ we have by Lemma 28 , when $m_{i}=n_{j}$

$$
\varphi\left(A_{i} B_{j}\right)=\varphi\left(a_{m_{1}+\cdots m_{i-1}+1} b_{n_{1}+\cdots+n_{j}}\right) \cdots \varphi\left(a_{m_{1}+\cdots m_{i}} b_{n_{1}+\cdots+n_{j-1}+1}\right)
$$

and 0 when $m_{i} \neq n_{j}$. Thus for this $\pi$, assuming $m_{i}=n_{j}$ for all $(i, j) \in \pi$, we have

$$
\varphi_{\pi}\left(A_{1}, \ldots, A_{p}, B_{1}, \ldots B_{p}\right)=\varphi_{\tilde{\pi}}\left(a_{1}, \ldots, a_{m}, b_{1}, \ldots, b_{m}\right)
$$

where $\tilde{\pi} \in S p^{+}(m)$ is the spoke diagram obtained by matching up $m_{1}+\cdots+m_{i-1}+k$ with $n_{1}+\cdots n_{j}-k+1$.

For $\pi \in S p^{-}(p)$ and $(i, j) \in \pi$ we have by Lemma 28, when $m_{i}=n_{j}$

$$
\varphi\left(A_{i} B_{j}^{t}\right)=\varphi\left(a_{m_{1}+\cdots m_{i-1}+1} b_{n_{1}+\cdots+n_{j-1}+1}^{t}\right) \cdots \varphi\left(a_{m_{1}+\cdots m_{i}} b_{n_{1}+\cdots+n_{j}}\right)
$$


and 0 when $m_{i} \neq n_{j}$. Thus for this $\pi$, assuming $m_{i}=n_{j}$ for all $(i, j) \in \pi$, we have

$$
\varphi_{\pi}\left(A_{1}, \ldots, A_{p}, B_{1}^{t}, \ldots B_{p}^{t}\right)=\varphi_{\tilde{\pi}}\left(a_{1}, \ldots, a_{m}, b_{1}^{t}, \ldots, b_{m}^{t}\right)
$$

where $\tilde{\pi} \in S p^{+}(m)$ is the spoke diagram obtained by matching up $m_{1}+\cdots+m_{i-1}+k$ with $n_{1}+\cdots n_{j-1}+k$.

If we let $\pi$ run over $\mathrm{Sp}^{+}(\mathrm{m})$ on the right hand side of (8), the corresponding $\tilde{\pi}$ 's will not exhaust all $\pi$ 's on the right hand side of $(7)$, but the ones that are missed are such that $\varphi_{\pi}\left(a_{1}, \ldots, a_{m}, b_{1}, \ldots, b_{m}\right)=0$, by the first order freeness of the $\mathcal{A}_{i}$ 's. Similarly for the $\pi$ 's in $S p^{-}(m)$ on the right hand side of (8). We thus have

$$
\begin{aligned}
\sum_{\pi \in S p^{+}(p)} \varphi_{\pi}\left(A_{1}, \ldots, A_{p}, B_{1}, \ldots, B_{p}\right) & \\
+ & \sum_{\pi \in S p^{-}(p)} \varphi_{\pi}\left(A_{1}, \ldots, A_{p}, B_{1}^{t}, \ldots, B_{p}^{t}\right) \\
& =\sum_{\pi \in S p^{+}(m)} \varphi_{\pi}\left(a_{1} \cdots a_{m}, b_{1} \cdots b_{m}\right) \\
& +\sum_{\pi \in S p^{-}(m)} \varphi_{\pi}\left(a_{1} \cdots a_{m}, b_{1}^{t} \cdots b_{m}^{t}\right) .
\end{aligned}
$$

This combined with (8) proves (7).

Definition 30. Suppose for each $d$ we have random matrices $\left\{A_{d, 1}, \ldots\right.$, $\left.A_{d, s}\right\}$. We say that the ensemble has a real second order limit distribution if there is a real second order non-commutative probability space $\left(\mathcal{A}, \varphi, \varphi_{2}, t\right)$ and $a_{1}, \ldots, a_{s} \in \mathcal{A}$ such that for all polynomials $p_{1}, p_{2}, p_{3}, \ldots$ in the non-commuting variables $\left\{x_{1}, \ldots, x_{s}, x_{1}^{t}, \ldots, x_{s}^{t}\right\}$ we have

i) $\lim _{d \rightarrow \infty} \mathrm{E}\left(\operatorname{tr}\left(p_{1}\left(A_{d, 1}, \ldots, A_{d, s}\right)\right)\right)=\varphi\left(p_{1}\left(a_{1}, \ldots, a_{s}\right)\right) ;$

$$
\lim _{d \rightarrow \infty} \operatorname{cov}\left(\operatorname{Tr}\left(p_{1}\left(A_{d, 1}, \ldots, A_{d, s}\right)\right),\right.
$$

iii) for all $r \geq 3$

$$
\begin{aligned}
& \left.\operatorname{Tr}\left(p_{2}\left(A_{d, 1}, \ldots, A_{d, s}\right)\right)\right) \\
& \quad=\varphi_{2}\left(p_{1}\left(a_{1}, \ldots, a_{s}\right), p_{2}\left(a_{1}, \ldots, a_{s}\right)\right)
\end{aligned}
$$

$$
\lim _{d \rightarrow \infty} k_{r}\left(\operatorname{Tr}\left(p_{1}\left(A_{d, 1}, \ldots, A_{d, s}\right)\right), \ldots, \operatorname{Tr}\left(p_{r}\left(A_{d, 1}, A_{d, 1}^{t}, \ldots, A_{d, s}\right)\right)\right)=0 .
$$

Remark 31. The third condition is only needed to ensure the convergence of fluctuations of mixed moments. In fact boundedness would be 
enough. For many ensembles of matrices the $r^{\text {th }}$ cumulant vanishes on the order of $d^{2-r}$, for example the ensembles discussed in $\left[\mathrm{R}_{1}, \mathrm{R}_{2}\right]$. For deterministic matrices the higher cumulants of traces are 0 . Moreover a close reading of our proof shows that if one starts with an ensemble $\left\{A_{i}\right\}_{i}$ with $k_{r}$ between $\mathrm{o}(1)$ and $\mathrm{O}(1)$ for $r \geq 3$, the mixed cumulants of $A$ 's and $O$ 's for $r \geq 3$ would have the same order as $\left\{A_{i}\right\}_{i}$.

Remark 32. Suppose we have for each $d$, random matrices $\left\{A_{d, 1}, \ldots\right.$, $\left.A_{d, s}\right\}$, a non-commutative probability space $(\mathcal{A}, \varphi)$, and $a_{1}, \ldots, a_{n} \in$ $\mathcal{A}$ such that for every polynomial $p$ in the non-commuting variables $x_{1}, \ldots, x_{s}, x_{1}^{t}, \ldots, x_{s}^{t}$ we have

$$
\lim _{d \rightarrow \infty} \operatorname{tr}\left(p\left(A_{d, 1}, \ldots, A_{d, n}\right)\right)=\varphi\left(p\left(a_{1}, \ldots, a_{n}\right)\right)
$$

then we say that the matrices $\left\{A_{d, 1}, \ldots, A_{d, n}\right\}$ have the limit joint $t$ distribution given by $a_{1}, \ldots, a_{n}$.

Definition 33. Let $\left\{A_{d, 1}, \ldots, A_{d, r}\right\}_{d}$ and $\left\{B_{d, 1} \ldots, B_{d, s}\right\}_{d}$ be two ensembles of random matrices such that $\left\{A_{d, 1}, \ldots, A_{d, r}, B_{d, 1} \ldots, B_{d, s}\right\}_{d}$ has a real second order limit distribution given by $\left\{a_{1}, \ldots, a_{s}, b_{1}, \ldots\right.$, $\left.b_{s}\right\}$ in the real second order non-commutative probability space $(\mathcal{A}, \varphi$, $\left.\varphi_{2}, t\right)$. If the two unital subalgebras $\mathcal{A}_{1}=\operatorname{alg}\left(1, a_{1}, \ldots, a_{r}, a_{1}^{t}, \ldots, a_{r}^{t}\right)$ and $\mathcal{A}_{2}=\operatorname{alg}\left(1, b_{1}, \ldots, b_{s}, b_{1}^{t}, \ldots, b_{s}^{t}\right)$ are real free of second order then we say that the two ensembles $\left\{A_{d, 1}, \ldots, A_{d, r}\right\}_{d}$ and $\left\{B_{d, 1} \ldots, B_{d, s}\right\}_{d}$ are asymptotically real free of second order.

\section{First Order Freeness of HaAr Orthogonal AND INDEPENDENT MATRICES}

To show that a family of $d \times d$ random matrices $\left\{A_{1}, \ldots, A_{s}\right\}_{d}$ and an independent family of orthogonal matrices $\left\{O_{d}\right\}_{d}$ are asymptotically real free of second order, we must first demonstrate that they are asymptotically free of first order, or asymptotically free in the sense of Voiculescu [VDN, §2.5].

For this we must show that given polynomials $\left\{p_{1}, \ldots, p_{n}\right\}$ in $O$ and $O^{-1}$ such that $\mathrm{E}\left(\operatorname{tr}\left(p_{i}\left(O, O^{-1}\right)\right)\right)=0$ and random matrices $\left\{A_{1}, \ldots\right.$, $\left.A_{s}\right\}$ with $\mathrm{E}\left(\operatorname{tr}\left(A_{i}\right)\right)=0$, then

$$
\lim _{d \rightarrow \infty} \mathrm{E}\left(\operatorname{tr}\left(p_{1}\left(O, O^{-1}\right) A_{1} \cdots p_{n}\left(O, O^{-1}\right) A_{s}\right)\right)=0
$$

provided that the entries of the $A_{d, i}$ 's are independent from those of the $O$ 's and the $\left\{A_{d, 1}, \ldots, A_{d, n}\right\}$ have a real second order limit distribution. For this it suffices to prove that

$$
\lim _{d \rightarrow \infty} \mathrm{E}\left(\operatorname{tr}\left(O^{m_{1}} A_{1} \cdots O^{m_{n}} A_{s}\right)\right)=0
$$


for any sequence of non-zero integers $m_{1}, \ldots, m_{n}$ and $\left\{A_{1}, \ldots, A_{s}\right\}$ as above.

Notation 34. Let $\pi \in S_{n}$ be a permutation and $\mathcal{U} \in \mathcal{P}(n)$ be a partition such that each cycle of $\pi$ lies in some block of $\mathcal{U}$. We denote this relation by $\pi \leq \mathcal{U}$. Let $A_{1}, \ldots, A_{n}$ be $d \times d$ random matrices and write, as in equation (3),

$$
\operatorname{Tr}_{\pi}\left(A_{1}, \ldots, A_{n}\right)=\sum_{i_{1}, \ldots, i_{n}}^{d} a_{i_{1} i_{\pi(1)}}^{(1)} \cdots a_{i_{n} i_{\pi(n)}}^{(n)}
$$

Let the blocks of $\mathcal{U}$ be $\left\{U_{1}, \ldots, U_{k}\right\}$ and let $\pi_{i}$ be the product of cycles of $\pi$ that lie in $U_{i}$. If $c=\left(i_{1}, \ldots, i_{r}\right)$ is a cycle of $\pi$, let $\operatorname{Tr}_{c}\left(A_{1}, \ldots, A_{n}\right)=\operatorname{Tr}\left(A_{i_{1}} \cdots A_{i_{r}}\right)$. If $\pi_{i}=c_{1} \cdots c_{k}$, as a product of cycles, let $\operatorname{Tr}_{\pi_{i}}\left(A_{1}, \ldots, A_{n}\right)=\prod_{i} \operatorname{Tr}_{c_{i}}\left(A_{1}, \ldots, A_{n}\right)$. Next let

$$
\mathrm{E}_{\mathcal{U}}\left(\operatorname{Tr}_{\pi}\left(A_{1}, \ldots, A_{n}\right)\right)=\prod_{i=1}^{k} \mathrm{E}\left(\operatorname{Tr}_{\pi_{i}}\left(A_{1}, \ldots, A_{n}\right)\right)
$$

Finally for $\eta=\left(\eta_{1}, \eta_{2}, \ldots, \eta_{n}\right) \in \mathbb{Z}_{2}^{n}$ and $\pi \in S_{n}$, let

$$
\mathrm{E}_{\mathcal{U}}\left(\operatorname{Tr}_{(\pi, \eta)}\left(A_{1}, \ldots, A_{n}\right)\right)=\mathrm{E}_{\mathcal{U}}\left(\operatorname{Tr}_{\pi}\left(A_{1}^{\left(\eta_{1}\right)}, \ldots, A_{n}^{\left(\eta_{n}\right)}\right)\right) .
$$

To make this clear let us give an example. Let $n=6, \pi=(1)(2,4)(3)$ and $\mathcal{U}=\{(1,3),(2,4)\}$. Then

$$
\mathrm{E}_{\mathcal{U}}\left(\operatorname{Tr}_{\pi}\left(A_{1}, A_{2}, A_{3}, A_{4}\right)\right)=\mathrm{E}\left(\operatorname{Tr}\left(A_{1}\right) \operatorname{Tr}\left(A_{3}\right)\right) \mathrm{E}\left(\operatorname{Tr}\left(A_{2} A_{4}\right)\right) .
$$

We shall also need to work with the normalized trace $\operatorname{tr}=d^{-1} \operatorname{Tr}$. We let $\operatorname{tr}_{\pi}\left(A_{1}, \ldots, A_{n}\right)=d^{-\#(\pi)} \operatorname{Tr}_{\pi}\left(A_{1}, \ldots, A_{n}\right)$.

If $\mathcal{U} \in \mathcal{P}(n)$ and $\pi \leq \mathcal{U}$, in the sense above, then we let

$$
k_{\mathcal{U}}\left(\operatorname{Tr}_{\pi}\left(A_{1}, \ldots, A_{n}\right)\right)=\sum_{\substack{\mathcal{V} \in \mathcal{P}(n) \\ \pi \leq \mathcal{V} \leq \mathcal{U}}} m(\mathcal{V}, \mathcal{U}) \mathrm{E}_{\mathcal{V}}\left(\operatorname{Tr}_{\pi_{i}}\left(A_{1}, \ldots, A_{n}\right)\right)
$$

Then by Möbius inversion we have

$$
\mathrm{E}_{\mathcal{U}}\left(\operatorname{Tr}_{\pi_{i}}\left(A_{1}, \ldots, A_{n}\right)\right)=\sum_{\substack{\mathcal{V} \in \mathcal{P}(n) \\ \pi \leq \mathcal{V} \leq \mathcal{U}}} k_{\mathcal{V}}\left(\operatorname{Tr}_{\pi}\left(A_{1}, \ldots, A_{n}\right)\right) .
$$

Remark 35. In what follows, for an ensemble of $d \times d$ matrices $\left\{A_{1}, \ldots\right.$, $\left.A_{s}\right\}_{d}$, will suppress the dependency of $A_{i}$ on $d$ and just denote it by $A_{i}$. Moreover the $(i, j)$-entry of $A_{k}$ will be denoted $a_{i j}^{(k)}$. This should not cause any confusion as at each stage of the discussion we shall only be multiplying matrices of the same size. Likewise for an ensemble of random orthogonal orthogonal matrices $\left\{O_{d}\right\}_{d}$, we shall drop the dependence on $d$ from the notation. 
Theorem 36. Let for each $d,\left\{A_{1}, \ldots, A_{n}\right\}$ be a ensemble of centred $d \times d$ random matrices that have a real second order limit distribution, $O$ a Haar distributed random $d \times d$ orthogonal matrix, and $m_{1}, \ldots, m_{n}$ be non-zero integers. Then

$$
\lim _{d \rightarrow \infty} \mathrm{E}\left(\operatorname{tr}\left(O^{m_{1}} A_{1} \cdots O^{m_{n}} A_{n}\right)\right)=0 .
$$

Proof. In order to be able to use the result of Proposition 12, with $\gamma=$ $(1,2,3 \ldots, n)$, we have to reduce it to the case of each $m_{i}$ being either 1 or -1 . We can achieve this by inserting an identity matrix, $I$, between any two adjacent $O$ 's or adjacent $O^{-1}$ 's. For example $O^{2} A_{1} O^{-1} A_{2}$ would become $O I O A_{1} O^{-1} A_{2}$. So with this change we must show that, whenever we have $\epsilon_{1}, \ldots, \epsilon_{n} \in\{-1,1\}$ and random matrices $A_{1}, \ldots, A_{n}$ with a limit joint $t$-distribution such that for each $i$, either $A_{i}$ is centred, i.e. $\mathrm{E}\left(\operatorname{tr}\left(A_{i}\right)\right)=0$, or $A_{i}=I$ and $\epsilon_{i}=\epsilon_{\gamma(i)}$, then

$$
\lim _{d \rightarrow \infty} \mathrm{E}\left(\operatorname{tr}\left(O^{\epsilon_{1}} A_{1} \cdots O^{\epsilon_{n}} A_{n}\right)\right)=0 .
$$

By Proposition 12

$$
\begin{aligned}
& \mathrm{E}\left(\operatorname{Tr}\left(O^{\epsilon_{1}} A_{1}, \ldots, O^{\epsilon_{n}} A_{n}\right)\right) \\
& \quad=\sum_{p, q \in \mathcal{P}_{2}(n)}\langle\operatorname{Wg}(p), q\rangle \mathrm{E}\left(\operatorname{Tr}_{\left.{\left(\pi_{p} \epsilon_{q}, \eta_{p} \cdot q\right.}\right)}\left(A_{1}, \ldots, A_{n}\right)\right) .
\end{aligned}
$$

Let us recall the construction of $\pi_{p \cdot{ }_{\epsilon} q}$. We write the permutation $\left(p \cdot{ }_{\epsilon} q\right) \delta$, which is the product of two pairings, as a product of cycles. We showed that the cycles always occur in pairs of the form $\left\{c, c^{\prime}\right\}$, where $c^{\prime}=\delta c^{-1} \delta$. From each pair we choose one, and then from this we obtained a cycle of $\pi_{p \cdot{ }_{\epsilon} q}$ by deleting any minus signs. The minus signs that are deleted are recorded in $\eta_{p_{\epsilon} q}$. So let us consider the singletons of $\pi_{p \cdot{ }_{\epsilon} q}$. If $(k)$ is a singleton of $\pi_{p \cdot{ }_{\epsilon} q}$, then $\left(p \cdot{ }_{\epsilon} q\right) \delta$ will have the two singletons $(k)(-k)$ and thus $(k,-k)$ will be a cycle of $\left(p \cdot{ }_{\epsilon} q\right)$ and hence $\left(-\delta_{\epsilon}(k), \delta_{\epsilon}(\gamma(k))\right)$ will be a cycle of $p \delta q \delta$. The cycles of $p \delta q \delta$ are either cycles of $p$, consisting of pairs of positive numbers, or cycles of $\delta q \delta$, consisting of pairs of negative numbers. Thus if $(k)$ is a singleton of $\pi_{p \cdot \epsilon q}$ then we must have $\epsilon_{k}=-\epsilon_{\gamma(k)}$, and hence $A_{k}$ is a centred matrix.

Now consider the expansion

$$
\begin{aligned}
& \mathrm{E}\left(\operatorname{Tr}\left(O^{\epsilon_{1}} A_{1}, \ldots, O^{\epsilon_{n}} A_{n}\right)\right) \\
& \quad=\sum_{p, q \in \mathcal{P}_{2}(n)}\langle\mathrm{Wg}(p), q\rangle \mathrm{E}\left(\operatorname{Tr}_{\left(\pi_{p \cdot \epsilon}, \eta_{p \epsilon q}\right)}\left(A_{1}, \ldots, A_{n}\right)\right) .
\end{aligned}
$$

We have

$$
\langle\mathrm{Wg}(p), q\rangle=\mathrm{O}\left(d^{-n+\#(p \vee q)}\right)
$$


We must next find a upper bound for the order of

$$
\mathrm{E}\left(\operatorname{Tr}_{\left(\pi_{p \cdot \epsilon q}, \eta_{p \cdot \epsilon q}\right)}\left(A_{1}, \ldots, A_{n}\right)\right)=\sum_{\pi \leq \mathcal{U}} k_{\mathcal{U}}\left(\operatorname{Tr}_{\left(\pi_{p \cdot \epsilon q}, \eta_{p \cdot \epsilon q}\right)}\left(A_{1}, \ldots, A_{n}\right)\right) .
$$

Since $\left\{A_{1}, \ldots, A_{n}\right\}$ has a real second order limit distribution we have that

$$
k_{\mathcal{U}}\left(\operatorname{Tr}_{\left(\pi_{p \cdot \epsilon q}, \eta_{p \cdot \epsilon q}\right)}\left(A_{1}, \ldots, A_{n}\right)\right)=\mathrm{O}\left(d^{u}\right)
$$

where $u$ is the number of blocks of $\mathcal{U}$ that contain a single cycle of $\pi$. If $\mathcal{U}$ has a singleton $(k)$ then $\pi$, too, will have a singleton $(k)$ and then $A_{k}$ will be centred so $k_{\mathcal{U}}\left(\operatorname{Tr}_{\left(\pi_{p \cdot \epsilon q}, \eta_{p \cdot \epsilon q}\right)}\left(A_{1}, \ldots, A_{n}\right)\right)$ will have a factor $\mathrm{E}\left(\operatorname{Tr}\left(A_{k}\right)\right)=0$, hence $k_{\mathcal{U}}\left(\operatorname{Tr}_{\left(\pi_{p \cdot \epsilon q}, \eta_{p \cdot \epsilon q}\right)}\left(A_{1}, \ldots, A_{n}\right)\right)=0$.

Thus $u \leq \#(\mathcal{U}) \leq n / 2$ and so $-n+\#(p \vee q)+u \leq 0$, thus

$$
\langle\mathrm{Wg}(p), q\rangle \mathrm{E}\left(\operatorname{Tr}_{\left(\pi_{p \cdot \epsilon q}, \eta_{p \cdot \epsilon q}\right)}\left(A_{1}, \ldots, A_{n}\right)\right)=\mathrm{O}(1) \text {. }
$$

Thus

$$
\mathrm{E}\left(\operatorname{Tr}\left(O^{\epsilon_{1}} A_{1}, \ldots, O^{\epsilon_{n}} A_{n}\right)\right)=\mathrm{O}(1)
$$

and hence

$$
\lim _{d \rightarrow \infty} \mathrm{E}\left(\operatorname{tr}\left(O^{\epsilon_{1}} A_{1}, \ldots, O^{\epsilon_{n}} A_{n}\right)\right)=0 .
$$

Corollary 37. Let $\left\{A_{1}, \ldots, A_{n+1}\right\}$ be $d \times d$ random matrices whose entries have moments of all orders, $O$ a Haar distributed random $d \times d$ orthogonal matrix, independent from $\left\{A_{1}, \ldots, A_{n+1}\right\}$, and $\epsilon_{1}, \ldots, \epsilon_{n} \in$ $\mathbb{Z}_{2}$. Suppose that for each $1 \leq i \leq n$ we have that either $\mathrm{E}\left(\operatorname{Tr}\left(A_{i}\right)\right)=0$ or $A_{i}=I$ and $\epsilon_{i}=\epsilon_{i+1}$ (using $\left.\epsilon_{n+1}=\epsilon_{1}\right)$, and $\mathrm{E}\left(\operatorname{Tr}\left(A_{n+1}\right)\right)=0$. Then

$$
\mathrm{E}\left(\operatorname{Tr}\left(O^{\epsilon_{1}} A_{1} \cdots O^{\epsilon_{n}} A_{n}\right)\right)=\mathrm{O}(1) \text {, }
$$

in fact

$$
\begin{aligned}
& \mathrm{E}\left(\operatorname{Tr}\left(O^{\epsilon_{1}} A_{1} \cdots O^{\epsilon_{n}} A_{n}\right)\right) \\
& \quad=d^{-n / 2} \sum_{p \in \mathcal{P}_{2}(n)} \mathrm{E}_{\pi_{p \cdot \epsilon}}\left(\operatorname{Tr}_{\left(\pi_{p \cdot \epsilon}, \eta_{p \cdot \epsilon}\right)}\left(A_{1}, \ldots, A_{n}\right)\right)+\mathrm{O}\left(d^{-1}\right)
\end{aligned}
$$

where the sum is over all $p$ 's such that $\pi_{p \cdot{ }_{\epsilon} p}$ is a pairing and

$$
\mathrm{E}\left(\operatorname{Tr}\left(O^{\epsilon_{1}} A_{1} \cdots O^{\epsilon_{n}} A_{n}\right) \operatorname{Tr}\left(A_{n+1}\right)\right)=\mathrm{O}\left(d^{-1}\right) .
$$

Proof. The first claim is just the second last equation of the proof of Theorem 36. Recall that when we expand into cumulants

$$
\mathrm{E}\left(\operatorname{Tr}_{\left(\pi_{p \cdot \epsilon q}, \eta_{p \cdot \epsilon q}\right)}\left(A_{1}, \ldots, A_{n}\right)\right)=\sum_{\substack{\mathcal{U} \in \mathcal{P}(n) \\ \pi_{p \cdot \epsilon q} \leq \mathcal{U}}} k_{\mathcal{U}}\left(A_{1}, \ldots, A_{n}\right)
$$

and let $u$ be the number of blocks of $\mathcal{U}$ that contain a single cycle of $\pi_{p \cdot{ }_{\epsilon} q}$ we have $-n+\#(p \vee q)+u \leq 0$ with equality only when $p=q$ 
and $n=n / 2$, i.e. $\mathcal{U}=\pi_{p \cdot{ }_{\epsilon} p}$ and $\pi_{p \cdot{ }_{\epsilon} p}$ is a pairing. This establishes the second claim.

By Proposition 14 we have

$$
\begin{gathered}
\mathrm{E}\left(\operatorname{Tr}\left(O^{\epsilon_{1}} A_{1} \cdots O^{\epsilon_{n}} A_{n}\right) \operatorname{Tr}\left(A_{n+1}\right)\right) \\
=\sum_{p, q \in \mathcal{P}_{2}(n)}\langle\mathrm{Wg}(p), q\rangle \mathrm{E}\left(\operatorname{Tr}_{\left(\pi_{p \cdot \epsilon q}, \eta_{p \cdot \epsilon q}\right)}\left(A_{1}, \ldots, A_{n}\right) \operatorname{Tr}\left(A_{n+1}\right)\right) .
\end{gathered}
$$

For the moment let us fix $p, q \in \mathcal{P}_{2}(n)$ and let $\tilde{\pi} \in S_{n+1}$ be the permutation which fixes $n+1$ and whose restriction to $[n]$ is $\pi_{p \cdot{ }_{\epsilon} q}$. Likewise let $\left.\tilde{\eta}\right|_{[n]}=\eta_{p_{\epsilon} q}$ and $\tilde{\eta}_{n+1}=1$. Then $\mathrm{E}\left(\operatorname{Tr}_{\left(\pi_{p \cdot \epsilon q}, \eta_{p \cdot \epsilon q}\right)}\left(A_{1}, \ldots, A_{n}\right) \operatorname{Tr}\left(A_{n+1}\right)\right)$

$=\mathrm{E}\left(\operatorname{Tr}_{(\tilde{\pi}, \tilde{\eta})}\left(A_{1}, \ldots, A_{n+1}\right)\right)$ Then we expand as above

$$
\mathrm{E}\left(\operatorname{Tr}_{(\tilde{\pi}, \tilde{\eta})}\left(A_{1}, \ldots, A_{n+1}\right)\right)=\sum_{\substack{\mathcal{U} \in \mathcal{P}(n+1) \\ \tilde{\pi} \leq \mathcal{U}}} k_{\mathcal{U}}\left(\operatorname{Tr}_{(\tilde{\pi}, \tilde{\eta})}\left(A_{1}, \ldots, A_{n+1}\right)\right) .
$$

Suppose $\mathcal{U} \in \mathcal{P}(n+1)$ is such that $\tilde{\pi} \leq \mathcal{U}$ and $k_{\mathcal{U}}\left(\operatorname{Tr}_{(\tilde{\pi}, \tilde{\eta})}\left(A_{1}, \ldots\right.\right.$, $\left.\left.A_{n+1}\right)\right) \neq 0$. Then

$$
k_{\mathcal{U}}\left(\operatorname{Tr}_{(\tilde{\pi}, \tilde{\eta})}\left(A_{1}, \ldots, A_{n+1}\right)\right)=\mathrm{O}\left(d^{u}\right)
$$

where $u$ is the number of blocks of $\mathcal{U}$ that contain only one cycle of $\tilde{\pi}$. Since, by assumption, $\mathrm{E}\left(\operatorname{Tr}\left(A_{n+1}\right)\right)=0$, the last cycle of $\tilde{\pi}$ cannot be in a block of $\mathcal{U}$ on its own (otherwise $k_{\mathcal{U}}=0$ ); thus $u \leq \#(\mathcal{U})-1$. As in the proof of Theorem 36. $\#\left(\left.\mathcal{U}\right|_{[n]}\right) \leq n / 2$ and as the cycle $(n+1)$ cannot be on its own we have $\#(\mathcal{U}) \leq n / 2$. So $u \leq n / 2-1$. Thus $-n+\#(p \vee q)+u \leq-1$ and so

$$
\langle\mathrm{Wg}(p), q\rangle k_{\mathcal{U}}\left(\operatorname{Tr}_{(\tilde{\pi}, \tilde{\eta})}\left(A_{1}, \ldots, A_{n+1}\right)\right)=\mathrm{O}\left(d^{-1}\right) .
$$

Since this holds for every $\mathcal{U}$ we have

$$
\langle\mathrm{Wg}(p), q\rangle \mathrm{E}\left(\operatorname{Tr}_{(\tilde{\pi}, \tilde{\eta})}\left(A_{1}, \ldots, A_{n+1}\right)\right)=\mathrm{O}\left(d^{-1}\right) .
$$

Since this in turn holds for every $p$ and $q$ we have

$$
\mathrm{E}\left(\operatorname{Tr}\left(O^{\epsilon_{1}} A_{1} \cdots O^{\epsilon_{n}} A_{n}\right) \operatorname{Tr}\left(A_{n+1}\right)\right)=\mathrm{O}\left(d^{-1}\right) .
$$

\section{Fluctuation Moments of Haar Orthogonal AND INDEPENDENT RANDOM MATRICES}

Our next step is to show that the limit distribution of Haar distributed orthogonal matrices and an independent ensemble of random matrices with a real second order limit distribution satisfies part (ii) (b) of Definition 25. Fix positive integers $m$ and $n$ and let $\gamma$ be the permutation with the two cycles $(1, \ldots, m)(m+1, \ldots, m+n)$. 
Theorem 38. Let $\left\{A_{1}, \ldots, A_{m}\right\}$ and $\left\{B_{1}, \ldots, B_{n}\right\}$ be a ensemble of centred $d \times d$ matrices that have a real second order limit distributions given by $\left(a_{1}, \ldots, a_{m}\right)$ and $\left(b_{1}, \ldots, b_{n}\right)$, respectively, in a real second order non-commutative probability space $\left(\mathcal{A}, \varphi, \varphi_{2}, t\right)$, and $O$ a Haar distributed random $d \times d$ orthogonal matrix, and $k_{1}, \ldots, k_{m}, l_{1}, \ldots, l_{n}$ nonzero integers. Suppose that the entries of $\left\{A_{1}, \ldots, A_{m}, B_{1}, \ldots, B_{n}\right\}$ are independent from those of $O$. Then

$$
\lim _{d \rightarrow \infty} \operatorname{cov}\left(\operatorname{Tr}\left(O^{k_{1}} A_{1} \cdots O^{k_{m}} A_{m}\right), \operatorname{Tr}\left(O^{l_{1}} B_{1} \cdots O^{l_{n}} B_{n}\right)\right)
$$

exists and equals 0 when $m \neq n$, and when $m=n \geq 2$, equals

$$
\sum_{r=1}^{m}\left\{\prod_{i=1}^{m} \varphi\left(a_{i} b_{r-i}\right) \varphi\left(o^{k_{i}+l_{r-(i-1)}}\right)+\prod_{i=1}^{m} \varphi\left(a_{i} b_{r+i}^{t}\right) \varphi\left(o^{k_{i}-l_{r+i}}\right)\right\} .
$$

where the indices of the b's and l's are taken modulo $m$.

Proof. We begin by noting that by Theorem $9, m+n$ must be even, otherwise the limit of the covariances is 0 . In order to apply Proposition 12 to the expression

$$
\operatorname{cov}\left(\operatorname{Tr}\left(O^{k_{1}} A_{1} \cdots O^{k_{m}} A_{m}\right), \operatorname{Tr}\left(O^{l_{1}} B_{1} \cdots O^{l_{n}} B_{n}\right)\right)
$$

we have to reduce it to the case where all $k$ 's and $l$ 's are either 1 or -1 . So let us consider the term $\varphi\left(a_{i} b_{r-i}\right) \varphi\left(o^{k_{i}+l_{r-(i-1)}}\right)$ of expression (13). In order for this to be non-zero we must have $k_{i}+l_{r-(i-1)}=$ 0 . So when we perform the reduction used in the proof of Theorem 36 we replace $o^{k_{i}}$, supposing $k_{i}>0$, with $o 1 o \cdots o 1 o$ and $o^{l_{r-i+1}}$ with $o^{-1} 1 o^{-1} \cdots o^{-1} 1 o^{-1}$ the factor $\varphi\left(o^{k_{i}+l_{r-i+1}}\right)=1$ gets replaced by $\varphi\left(o o^{-1}\right) \varphi(11) \varphi\left(o o^{-1}\right) \cdots \varphi\left(o o^{-1}\right) \varphi(11) \varphi\left(o o^{-1}\right)=1$. Likewise with the factor $\varphi\left(o^{k_{i}-l_{r+i}}\right)$. Thus without loss of generality we can assume that $k_{1} \ldots, k_{m} l_{1}, \ldots, l_{n} \in\{-1,1\}$. In this case we must show that

$$
\lim _{d \rightarrow \infty} \operatorname{cov}\left(\operatorname{Tr}\left(O^{\epsilon_{1}} A_{1} \cdots O^{\epsilon_{m}} A_{m}\right), \operatorname{Tr}\left(O^{\epsilon_{m+1}} B_{1} \cdots O^{\epsilon_{m+n}} B_{n}\right)\right)
$$

exists and equals 0 when $m \neq n$ and when $m=n$ equals

$$
\sum_{r=1}^{m}\left\{\prod_{i=1}^{m} \varphi\left(a_{i} b_{r-i}\right) \delta_{\epsilon_{i},-\epsilon_{\gamma^{-i+1}(m+r)}}+\prod_{i=1}^{m} \varphi\left(a_{i} b_{r+i}^{t}\right) \delta_{\epsilon_{i}, \epsilon_{\gamma^{i}(m+r)}}\right\}
$$

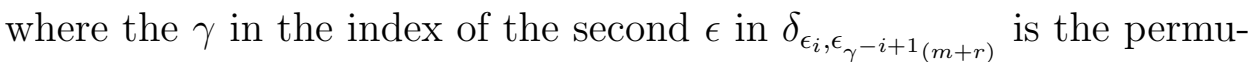
tation with cycle decomposition $(1, \ldots, m)(m+1, \ldots, 2 m)$.

By Proposition 12

$$
\begin{aligned}
& \mathrm{E}\left(\operatorname{Tr}\left(O^{\epsilon_{1}} A_{1} \cdots O^{\epsilon_{m}} A_{m}\right) \operatorname{Tr}\left(O^{\epsilon_{m+1}} B_{1} \cdots O^{\epsilon_{m+n}} B_{n}\right)\right) \\
= & \sum_{p, q \in \mathcal{P}_{2}(m+n)}\langle\mathrm{Wg}(p), q\rangle \mathrm{E}\left(\operatorname{Tr}_{\left(\pi_{p \cdot \epsilon q}, \eta_{p \cdot \epsilon}\right)}\left(A_{1}, \ldots, B_{n}\right)\right),
\end{aligned}
$$


and

$$
\begin{gathered}
\mathrm{E}\left(\operatorname{Tr}\left(O^{\epsilon_{1}} A_{1} \cdots O^{\epsilon_{m}} A_{m}\right)\right) \mathrm{E}\left(\operatorname{Tr}\left(O^{\epsilon_{m+1}} B_{1} \cdots O^{\epsilon_{m+n}} B_{n}\right)\right) \\
=\sum_{\substack{p, q \in \mathcal{P}_{2}(m+n) \\
p, q \leq \gamma}} \operatorname{Wg}(\gamma, p, q) \mathrm{E}_{\gamma}\left(\operatorname{Tr}_{\pi_{p \cdot \epsilon}(}\left(\vec{A}^{\eta}, \vec{B}^{\eta}\right)\right) .
\end{gathered}
$$

To simplify the notation we let $\operatorname{Tr}_{\pi_{p \cdot \epsilon q}}\left(\overrightarrow{A^{\eta}}, \overrightarrow{B^{\eta}}\right)=\operatorname{Tr}_{\left(\pi_{p \cdot \epsilon q}, \eta_{p \cdot \epsilon q}\right)}\left(A_{1}, \ldots\right.$, $\left.B_{n}\right)$. Thus

$$
\begin{aligned}
& \operatorname{cov}\left(\operatorname{Tr}\left(O^{\epsilon_{1}} A_{1} \cdots O^{\epsilon_{m}} A_{m}\right), \operatorname{Tr}\left(O^{\epsilon_{m+1}} B_{1} \cdots O^{\epsilon_{m+n}} B_{n}\right)\right) \\
& =\sum_{p, q \in \mathcal{P}_{2}(m+n)}\langle\operatorname{Wg}(p), q\rangle \mathrm{E}\left(\operatorname{Tr}_{\pi_{p \cdot \epsilon}}\left(\vec{A}^{\eta}, \vec{B}^{\eta}\right)\right) \\
& \quad-\sum_{\substack{p, q \in \mathcal{P}_{2}(m+n) \\
p, q \leq \gamma}} \operatorname{Wg}(\gamma, p, q) \mathrm{E}_{\gamma}\left(\operatorname{Tr}_{\pi_{p \cdot \epsilon}}\left(\vec{A}^{\eta}, \vec{B}^{\eta}\right)\right) \\
& =\sum_{\substack{p, q \in \mathcal{P}_{2}(m+n) \\
p \vee q \vee \gamma=1_{m+n}}}\langle\operatorname{Wg}(p), q\rangle \mathrm{E}\left(\operatorname{Tr}_{\pi_{p \cdot \epsilon q}}\left(\vec{A}^{\eta}, \vec{B}^{\eta}\right)\right) \\
& \quad+\sum_{p, q \in \mathcal{P}_{2}(m+n)}\left\{\langle\operatorname{Wg}(p), q\rangle-\mathrm{Wg}_{p, q \leq \gamma}(\gamma, p, q)\right\} \mathrm{E}\left(\operatorname{Tr}_{\pi_{p \cdot \epsilon q}}\left(\vec{A}^{\eta}, \vec{B}^{\eta}\right)\right) \\
& \quad+\sum_{\substack{p, q \in \mathcal{P}_{2}(m+n) \\
p, q \leq \gamma}} \operatorname{Wg}(\gamma, p, q)\left\{\mathrm{E}\left(\operatorname{Tr}_{\pi_{p \cdot \epsilon q}}\left(\vec{A}^{\eta}, \vec{B}^{\eta}\right)\right)-\mathrm{E}_{\gamma}\left(\operatorname{Tr}_{\pi_{p \cdot \epsilon q}}\left(\vec{A}^{\eta}, \vec{B}^{\eta}\right)\right)\right\} .
\end{aligned}
$$

We shall show that the first term 15 converges to

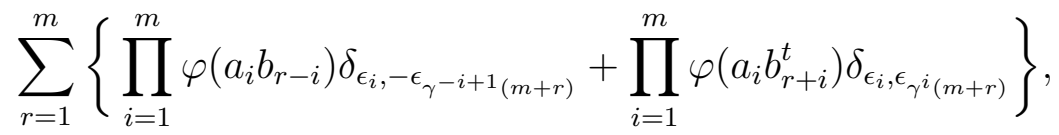

and the second (16) and third term (17) converge to 0.

We first consider expression (15), and show that this has the limit we have claimed. Let us find the order of $\mathrm{E}\left(\operatorname{Tr}_{\pi_{p \cdot \epsilon q}}\left(\overrightarrow{A^{\eta}}, \overrightarrow{B^{\eta}}\right)\right)$; to do this we have to rewrite this expectation in terms of cumulants so that we can use our assumptions about the $A$ 's and $B$ 's having a real second order limit distribution. If we consider $\pi_{p \cdot{ }_{\epsilon} q}$ a partition of $[m+n]$ then by equation (11) we have

$$
\begin{aligned}
& \mathrm{E}\left(\operatorname{Tr}_{\left(\pi_{p^{\prime} \epsilon}, \eta_{p^{\prime} \epsilon}\right)}\left(A_{1}, \ldots, B_{n}\right)\right) \\
& =\sum_{\substack{\mathcal{U} \in \mathcal{P}(m+n) \\
\mathcal{U} \geq \pi_{p \cdot \epsilon q}}} k_{\mathcal{U}}\left(\operatorname{Tr}_{\left(\pi_{p \cdot \epsilon}, \eta_{p \cdot \epsilon}\right)}\left(A_{1}, \ldots, B_{n}\right)\right) .
\end{aligned}
$$


Suppose $\mathcal{U} \in \mathcal{P}(m+n)$ and $\mathcal{U} \geq \pi_{p \cdot{ }_{\epsilon} q}$. If $\mathcal{U}$ has a singleton $(k)$, then $(k)$ is also a singleton of $\pi_{p \cdot{ }_{\epsilon} q}$. As in the proof of Theorem 36, this implies that $A_{k}$ (or $B_{k-m}$ if $\left.k>m\right)$ is centred, and thus, $k_{\mathcal{U}}\left(\operatorname{Tr}_{\pi_{p_{\epsilon} q}}\left(\overrightarrow{A^{\eta}}, \overrightarrow{B^{\eta}}\right)\right)=$ 0 . Thus we only have to consider $\mathcal{U}$ 's with no singletons. Hence $\#(\mathcal{U}) \leq$ $(m+n) / 2$. Suppose $U$ is a block of $\mathcal{U}$ which contains two or more cycles of $\pi_{p{ }_{\epsilon} q}$; the corresponding factor in Equation (18) is a second or higher cumulant of traces, which converge by our assumption that the $A$ 's and $B$ 's have a real second order limit distribution. Hence these factors will be of order $\mathrm{O}\left(d^{0}\right)$. Each block of $\mathcal{U}$ which contains only one cycle of $\pi_{p \cdot{ }_{\epsilon} q}$ will be of order $\mathrm{O}(d)$. Hence $k_{\mathcal{U}}\left(\operatorname{Tr}_{\pi_{p \cdot \epsilon q}}\left(\overrightarrow{A^{\eta}}, \overrightarrow{B^{\eta}}\right)\right)=\mathrm{O}\left(d^{u}\right)$ where $u$ is the number of blocks of $\mathcal{U}$ which contain only one cycle of $\pi_{p{ }_{\epsilon} q}$. As

$$
u \leq \#(\mathcal{U}) \leq(m+n) / 2,
$$

we have $k_{\mathcal{U}}\left(\operatorname{Tr}_{\pi_{p \cdot \epsilon q}}\left(\overrightarrow{A^{\eta}}, \overrightarrow{B^{\eta}}\right)\right)=\mathrm{O}\left(d^{(m+n) / 2}\right)$ and the order $(m+n) / 2$ can only be achieved when $u=(m+n) / 2$, which implies that $\pi_{p \cdot{ }_{\epsilon} q}=\mathcal{U}$, as partitions, and no cycle of $\pi_{p_{\epsilon_{\epsilon}} q}$ is a singleton, because no block of $\mathcal{U}$ is a singleton. If $\#\left(\pi_{p{ }_{\epsilon} q}\right)=u=(m+n) / 2$ and $\pi_{p{ }_{\epsilon} q}$ has no singletons; $\pi_{p{ }_{\epsilon} q}$ must be a pairing. Combining these conclusions we have

$$
\mathrm{E}\left(\operatorname{Tr}_{\pi_{p \cdot \epsilon q}}\left(\overrightarrow{A^{\eta}}, \overrightarrow{B^{\eta}}\right)\right)=\mathrm{O}\left(d^{(m+n) / 2-1}\right)
$$

unless $p=q$ and $\pi_{p \cdot{ }_{\epsilon} q}$ is a pairing, in which case

$$
\begin{aligned}
& \mathrm{E} \\
& \left.\quad \operatorname{Tr}_{\pi_{p_{\epsilon} \epsilon}}\left(\vec{A}^{\eta}, \vec{B}^{\eta}\right)\right) \\
& \quad=\mathrm{E}_{\pi_{p^{\prime} \epsilon q}}\left(\operatorname{Tr}_{\pi_{p_{\epsilon} q}}\left(\vec{A}^{\eta}, \vec{B}^{\eta}\right)\right)+\mathrm{O}\left(d^{(m+n) / 2-1}\right) .
\end{aligned}
$$

Using our usual bound on the order of $\mathrm{Wg}$, namely

$$
\langle\mathrm{Wg}(p), q\rangle=\mathrm{O}\left(d^{-(m+n)+\#(p \vee q)}\right),
$$

we thus have

$$
\langle\mathrm{Wg}(p), q\rangle \mathrm{E}\left(\operatorname{Tr}_{\pi_{p \cdot \epsilon q}}\left(\vec{A}^{\eta}, \vec{B}^{\eta}\right)\right)=\mathrm{O}\left(d^{-1}\right)
$$

unless $p=q$ and $\pi_{p{ }_{\epsilon} q}$ is a pairing, in which case

$$
\begin{aligned}
& \langle\mathrm{Wg}(p), q\rangle \mathrm{E}\left(\operatorname{Tr}_{\pi_{p \cdot \epsilon q}}\left(\overrightarrow{A^{\eta}}, \overrightarrow{B^{\eta}}\right)\right) \\
& \quad=\mathrm{E}_{\pi_{p \cdot \epsilon q}}\left(\operatorname{tr}_{\pi_{p^{\prime} \epsilon}}\left(\vec{A}^{\eta}, \vec{B}^{\eta}\right)\right)+\mathrm{O}\left(d^{-1}\right) .
\end{aligned}
$$

Thus

$$
\begin{aligned}
& \sum_{\substack{p, q \in \mathcal{P}_{2}(m+n) \\
p \vee q \vee \gamma=1_{m+n}}}\langle\mathrm{Wg}(p), q\rangle \mathrm{E}\left(\operatorname{Tr}_{\pi_{p \cdot \epsilon}}\left(\vec{A}^{\eta}, \vec{B}^{\eta}\right)\right) \\
= & \sum_{p \in \mathcal{P}_{2}(m+n)} \mathrm{E}_{\pi_{p \cdot \epsilon p}}\left(\operatorname{tr}_{\pi_{p \cdot \epsilon p}}\left(\vec{A}^{\eta}, \vec{B}^{\eta}\right)\right)+\mathrm{O}\left(d^{-1}\right)
\end{aligned}
$$


where the second sum runs over all $p$ such that $p \vee \gamma=1_{m+n}$ and $\pi_{p \cdot{ }_{\epsilon} p}$ is a pairing. To find the limit as $d \rightarrow \infty$ we use Lemmas 20 and 21 .

First suppose that there is $(u, v) \in p$ such that $\epsilon_{u}=-\epsilon_{v}$. Then by Lemma 20 we have $m=n$, every cycle of $p$ connects the two cycles of $\gamma$, and $\epsilon_{i}=-\epsilon_{j}$ for all $(i, j) \in p$. Then for some $r \in[m]$ we have $(m-1, m+r) \in p$. Again by Lemma 20 we have for all $k \in[m]$

$\circ\left(k, \gamma^{-k}(m+r)\right) \in \pi_{p \cdot \epsilon}$,

$\circ\left(k, \gamma^{-k+1}(m+r)\right) \in p$,

$\circ \eta_{k}=1$.

Thus $\epsilon_{k}=-\epsilon_{\gamma^{-k+1}(m+r)}$ and

$\mathrm{E}_{\pi_{p \cdot \epsilon p}}\left(\operatorname{tr}_{\left(\pi_{p \cdot \epsilon}, \eta_{p \cdot \epsilon}\right)}\left(A_{1}, \ldots, B_{m}\right)\right)=\prod_{k=1}^{m} \mathrm{E}\left(\operatorname{tr}\left(A_{k} B_{r-k}\right)\right) \delta_{\epsilon_{k},-\epsilon_{\gamma^{-i+1}}(m+r)}$

which converges to

$$
\prod_{k=1}^{m} \varphi\left(a_{k} b_{r-k}\right) \delta_{\epsilon_{k},-\epsilon_{\gamma-k+1}(m+r)}
$$

as $d \rightarrow \infty$.

Next suppose that there is $(u, v) \in p$ such that $\epsilon_{u}=\epsilon_{v}$. Then by Lemma 21 we have $m=n$, every cycle of $p$ connects the two cycles of $\gamma$, and $\epsilon_{i}=\epsilon_{j}$ for all $(i, j) \in p$. Then for some $r \in[m]$ we have $(m-1, m+r) \in p$. As in Lemma 21, let $l=\gamma^{-m+1}(m+r)=\gamma^{r}(2 m)$. Then $\gamma^{k}(l)=\gamma^{r}(m+k)$, for $k \in[m]$. Hence by Lemma 21 we have for all $k \in[m]$

$$
\begin{aligned}
& \circ\left(k, \gamma^{r}(m+k)\right) \in \pi_{p \cdot{ }_{\epsilon} p}, \\
& \circ\left(k, \gamma^{r}(m+k)\right) \in p, \\
& \circ \eta_{k}=-1 .
\end{aligned}
$$

Thus $\epsilon_{k}=\epsilon_{\gamma^{k}(m+r)}$.

$$
\mathrm{E}_{\pi_{p \cdot \epsilon p}}\left(\operatorname{tr}_{\left(\pi_{p \cdot \epsilon}, \eta_{p \cdot \epsilon p}\right)}\left(A_{1}, \ldots, B_{m}\right)\right)=\prod_{k=1}^{m} \mathrm{E}\left(\operatorname{tr}\left(A_{k} B_{r+k}^{t}\right)\right) \delta_{\epsilon_{k}, \epsilon_{\gamma^{k}(m+r)}}
$$

which converges to

$$
\prod_{k=1}^{m} \varphi\left(a_{k} b_{r+k}\right) \delta_{\epsilon_{k}, \epsilon_{\gamma^{k}(m+r)}}
$$

as $d \rightarrow \infty$. Hence the expression 15 converges to

$$
\sum_{r=1}^{m}\left\{\prod_{k=1}^{m} \varphi\left(a_{k} b_{r-k}\right) \delta_{\epsilon_{k},-\epsilon_{\gamma-i+1}(m+r)}+\prod_{k=1}^{m} \varphi\left(a_{k} b_{r+k}^{t}\right) \delta_{\epsilon_{k}, \epsilon_{\gamma^{i}(m+r)}}\right\} .
$$


To show that (16) and (17) vanish as $d \rightarrow \infty$ we have to consider the order of $\mathrm{E}_{\gamma}\left(\operatorname{Tr}_{\pi_{p \cdot \epsilon}}\left(\overrightarrow{A^{\eta}}, \overrightarrow{B^{\eta}}\right)\right)$ with $p, q \leq \gamma$. As before we write this as a sum of cumulants

$$
\mathrm{E}_{\gamma}\left(\operatorname{Tr}_{\pi_{p \cdot \epsilon q}}\left(\vec{A}^{\eta}, \vec{B}^{\eta}\right)\right)=\sum_{\substack{\mathcal{U} \in \mathcal{P}(m+n) \\ \mathcal{U} \leq \gamma}} k_{\mathcal{U}}\left(\operatorname{Tr}_{\pi_{p \cdot \epsilon q}}\left(\vec{A}^{\eta}, \vec{B}^{\eta}\right)\right) .
$$

Let $u$ be the number of blocks of $\mathcal{U}$ that contain only one cycle of $\pi_{p{ }_{\epsilon} q}$. If $\mathcal{U}$ has a singleton then the corresponding cumulant will be 0 because the $A$ 's and $B$ 's are centred; so we only consider $\mathcal{U}$ 's which have no singletons and thus $\#(\mathcal{U}) \leq(m+n) / 2$. If we let $u$ be the number of blocks of $\mathcal{U}$ that contain exactly one cycle of $\pi_{p \cdot{ }_{\epsilon} q}$, then $k_{\mathcal{U}}\left(\operatorname{Tr}_{p{ }_{\epsilon} q}\left(\overrightarrow{A^{\eta}}, \vec{B}^{\eta}\right)\right)=\mathrm{O}\left(d^{u}\right)$ and $u \leq \#(\mathcal{U}) \leq(m+n) / 2$. Recall that $\langle\mathrm{Wg}(p), q\rangle-\mathrm{Wg}(\gamma, p, q)=\mathrm{O}\left(d^{-(m+n)+\#(p \vee q)-1}\right)$

Since $\#(p \vee q) \leq(m+n) / 2$ we have

$$
\begin{aligned}
\{\mathrm{Wg}(\gamma, p, q)- & \mathrm{Wg}(\gamma, p, q)\} k_{\mathcal{U}}\left(\operatorname{Tr}_{\pi_{p \cdot \epsilon} q}\left(\vec{A}^{\eta}, \vec{B}^{\eta}\right)\right) \\
= & \mathrm{O}\left(d^{-(m+n)+\#(p \vee q)-1+u}\right)=\mathrm{O}\left(d^{-1}\right) .
\end{aligned}
$$

Then summing over all $\mathcal{U}$ 's we have

$$
\{\mathrm{Wg}(\gamma, p, q)-\mathrm{Wg}(\gamma, p, q)\} \mathrm{E}_{\gamma}\left(\operatorname{Tr}_{\pi_{p \cdot \epsilon q}}\left(\overrightarrow{A^{\eta}}, \vec{B}^{\eta}\right)\right)=\mathrm{O}\left(d^{-1}\right) .
$$

Thus the expression 16

$$
\sum_{\substack{p, q \in \mathcal{P}_{2}(m+n) \\ p, q \leq \gamma}}\{\langle\mathrm{Wg}(p), q\rangle-\mathrm{Wg}(\gamma, p, q)\} \mathrm{E}\left(\operatorname{Tr}_{\pi_{p \cdot \epsilon q}}\left(\overrightarrow{A^{\eta}}, \vec{B}^{\eta}\right)\right) .
$$

converges to 0 .

Let us finally consider the expression (17)

$$
\sum_{\substack{p, q \in \mathcal{P}_{2}(m+n) \\ p, q \leq \gamma}} \operatorname{Wg}(\gamma, p, q)\left\{\mathrm{E}\left(\operatorname{Tr}_{\pi_{p_{\epsilon} \in q}}\left(\overrightarrow{A^{\eta}}, \overrightarrow{B^{\eta}}\right)\right)-\mathrm{E}_{\gamma}\left(\operatorname{Tr}_{\pi_{p \cdot \epsilon q}}\left(\overrightarrow{A^{\eta}}, \overrightarrow{B^{\eta}}\right)\right)\right\} .
$$

For each $p, q \leq \gamma$ we must show that

$$
\mathrm{Wg}(\gamma, p, q)\left\{\mathrm{E}\left(\operatorname{Tr}_{\pi_{p_{\epsilon} q}}\left(\overrightarrow{A^{\eta}}, \vec{B}^{\eta}\right)\right)-\mathrm{E}_{\gamma}\left(\operatorname{Tr}_{\pi_{p \cdot \epsilon q}}\left(\vec{A}^{\eta}, \vec{B}^{\eta}\right)\right)\right\}=\mathrm{O}\left(d^{-1}\right) .
$$

So fix $p, q \leq \gamma$ and write $\operatorname{Tr}_{\pi_{p \cdot \epsilon q}}\left(\overrightarrow{A^{\eta}}, \overrightarrow{B^{\eta}}\right)=X_{1} \cdots X_{r} X_{r+1} \cdots X_{r+s}$ with $X_{1}, \ldots, X_{r}$ coming from the cycles of $\pi_{p \cdot{ }_{\epsilon} q}$ contained in $[m]$ and $X_{r+1}, \ldots, X_{r+s}$ coming from the cycles of $\pi_{p{ }_{\epsilon} q}$ contained in $[m+1, m+$ $n]$. Then

$\mathrm{E}\left(\operatorname{Tr}_{\pi_{p \cdot \epsilon q}}\left(\overrightarrow{A^{\eta}}, \overrightarrow{B^{\eta}}\right)\right)-\mathrm{E}_{\gamma}\left(\operatorname{Tr}_{\pi_{p \cdot \epsilon q}}\left(\vec{A}^{\eta}, \vec{B}^{\eta}\right)\right)=k_{2}\left(X_{1} \cdots X_{r}, X_{r+1} \cdots X_{r+s}\right)$. 
Using the formula of Leonov and Shiryaev [LS]

$$
k_{2}\left(X_{1} \cdots X_{r}, X_{r+1} \cdots X_{r+s}\right)=\sum_{\substack{\mathcal{V} \in \mathcal{P}(r+s) \\ \mathcal{V} \vee \tau=1_{r+s}}} k_{\mathcal{V}}\left(X_{1}, \cdots, X_{r+s}\right)
$$

where $\tau=\{(1, \ldots, r)(r+1, \ldots, r+s)\}$. Now let us use Notation 34 to write this as

$$
\mathrm{E}\left(\operatorname{Tr}_{\pi_{p^{\prime} \epsilon q}}\left(\vec{A}^{\eta}, \vec{B}^{\eta}\right)\right)-\mathrm{E}_{\gamma}\left(\operatorname{Tr}_{\pi_{p_{\epsilon} \in}}\left(\vec{A}^{\eta}, \vec{B}^{\eta}\right)\right)=\sum_{\substack{\pi_{p \cdot \epsilon q} \leq \mathcal{U} \in \mathcal{P}(m+n) \\ \mathcal{U} \vee \gamma=1_{m+n}}} k_{\mathcal{U}}\left(\operatorname{Tr}_{\pi_{p \cdot \epsilon q}}\left(\overrightarrow{A^{\eta}}, \vec{B}^{\eta}\right)\right) .
$$

If $\mathcal{U}$ has a singleton $(k)$ then $\pi_{p \cdot{ }_{\epsilon} q}$ will have a singleton $(k)$. As in the proof of Theorem 36 this singleton must be a centred $A_{k}$ (or $B_{k-m}$ if $k>m)$. So if $\mathcal{U}$ has a singleton we must have $k_{\mathcal{U}}\left(\operatorname{Tr}_{\pi_{p \cdot \epsilon} q}\left(\overrightarrow{A^{\eta}}, \overrightarrow{B^{\eta}}\right)\right)=0$. Thus we may assume that $\mathcal{U}$ has no singletons, so in particular $\#(\mathcal{U}) \leq$ $(m+n) / 2$. . As before let $u$ be the number of blocks of $\mathcal{U}$ that contain exactly one cycle of $\pi_{p \cdot{ }_{\epsilon} q}$. Then

$$
k_{\mathcal{U}}\left(\operatorname{Tr}_{\pi_{p \cdot \epsilon q}}\left(\vec{A}^{\eta}, \overrightarrow{B^{\eta}}\right)\right)=\mathrm{O}\left(d^{u}\right) .
$$

Now $u \leq \#(\mathcal{U}) \leq(m+n) / 2$ and, as usual,

$$
\mathrm{Wg}(\gamma, p, q)=\mathrm{O}\left(d^{-(m+n)+\#(p \vee q)}\right) .
$$

Thus

$$
\mathrm{Wg}(\gamma, p, q) k_{\mathcal{U}}\left(\operatorname{Tr}_{\pi_{p \cdot \epsilon} q}\left(\vec{A}^{\eta}, \vec{B}^{\eta}\right)\right)=\mathrm{O}\left(d^{-(m+n)+\#(p \vee q)+u}\right) .
$$

Since $\pi_{p \cdot{ }_{\epsilon} q} \leq \gamma$ and $\mathcal{U} \vee \gamma=1_{m+n}$ we must have $u<(m+n) / 2$, as equality would force $\pi_{p{ }_{\epsilon} q}=\mathcal{U}$ as partitions. Thus $-(m+n)+\#(p \vee$ q) $+u \leq-1$. Hence

$$
\mathrm{Wg}(\gamma, p, q) k_{\mathcal{U}}\left(\operatorname{Tr}_{\pi_{p \cdot \epsilon q}}\left(\vec{A}^{\eta}, \vec{B}^{\eta}\right)\right)=\mathrm{O}\left(d^{-1}\right) .
$$

Summing over all $\mathcal{U}$ 's we have

$$
\mathrm{Wg}(\gamma, p, q)\left\{\mathrm{E}\left(\operatorname{Tr}_{\pi_{p \cdot \epsilon}}\left(\vec{A}^{\eta}, \vec{B}^{\eta}\right)\right)-\mathrm{E}_{\gamma}\left(\operatorname{Tr}_{\pi_{p \cdot \epsilon q}}\left(\vec{A}^{\eta}, \vec{B}^{\eta}\right)\right)\right\}=\mathrm{O}\left(d^{-1}\right) .
$$

Remark 39. The proof of Theorem 38 actually proves a stronger statement than was claimed. Let $A_{1}, \ldots, A_{s}$ is an ensemble of $d \times d$ centred random matrices where for $\eta \in\{-1,1\}$ we let $A^{\eta}=A^{t}$ for $\eta=-1$ we let $A^{\eta}=A_{j}$ for $\eta=1$. Suppose that for any monomials $W_{k}=A_{i_{1}, k}^{\eta_{i_{1}, k}} \cdots A_{i_{n_{k}}, k}^{\eta_{n_{k}, k}}$, we have

$\circ \mathrm{E}\left(\operatorname{tr}\left(W_{i}\right)\right)=\mathrm{O}\left(d^{0}\right)$ and

○ $k_{r}\left(\operatorname{Tr}\left(W_{i_{1}}\right), \ldots, \operatorname{Tr}\left(W_{i_{r}}\right)\right)=\mathrm{O}\left(d^{0}\right)$ for $r \geq 2$. 
Then by equation 20 we have for $m \neq n$

$$
\operatorname{cov}\left(\operatorname{Tr}\left(O^{\epsilon_{1}} A_{i_{1}} \cdots O^{\epsilon_{m}} A_{i_{m}}\right), \operatorname{Tr}\left(O^{\eta_{1}} A_{j_{1}} \cdots O^{\eta_{n}} A_{j_{n}}\right)\right)=\mathrm{O}\left(d^{-1}\right)
$$

and by equations $(21)$ and $(22)$ we have for $m=n$

$$
\begin{aligned}
\operatorname{cov}\left(\operatorname { T r } \left(O^{\epsilon_{1}} A_{i_{1}} \cdots\right.\right. & \left.\left.O^{\epsilon_{m}} A_{i_{m}}\right), \operatorname{Tr}\left(O^{\eta_{1}} A_{j_{1}} \cdots O^{\eta_{m}} A_{j_{m}}\right)\right) \\
=\sum_{s=1}^{m} & \left\{\prod_{r=1}^{m} \mathrm{E}\left(\operatorname{tr}\left(A_{i_{r}} A_{j_{s-r}}\right)\right) \mathrm{E}\left(\operatorname{tr}\left(O^{\epsilon_{r}+\eta_{s-r+1}}\right)\right)\right. \\
& \left.+\prod_{r=1}^{m} \mathrm{E}\left(\operatorname{tr}\left(A_{i_{r}} A_{j_{s+r}}^{t}\right)\right) \mathrm{E}\left(\operatorname{tr}\left(O^{\epsilon_{r}-\eta_{s-r}}\right)\right)\right\}+\mathrm{O}\left(d^{-1}\right),
\end{aligned}
$$

where the indices of the $j$ 's and $\eta$ 's are interpreted modulo $m$.

Corollary 40. Let $O$ be a $d \times d$ Haar distributed random orthogonal matrix. Then for integers $m$ and $n$

$$
\lim _{d \rightarrow \infty} \operatorname{cov}\left(\operatorname{Tr}\left(O^{m}\right), \operatorname{Tr}\left(O^{n}\right)\right)=\left\{\begin{array}{ll}
0 & |m| \neq|n| \\
2|m| & |m|=|n|
\end{array} .\right.
$$

Proof. Let $\epsilon_{1}=\cdots=\epsilon_{m}=\operatorname{sgn}(m)$ and $\epsilon_{m+1}=\cdots=\epsilon_{m+n}=$ $\operatorname{sgn}(n)$. Let $\gamma$ be the permutation with the two cycles $(1,2, \ldots, m)(m+$ $1, \ldots, m+n)$. Then by Proposition 12

$$
\mathrm{E}\left(\operatorname{Tr}\left(O^{\epsilon_{1}} \cdots O^{\epsilon_{m}}\right) \operatorname{Tr}\left(O^{\epsilon_{m+1}} \cdots O^{\epsilon_{m+n}}\right)\right)=\sum_{p, q \in \mathcal{P}_{2}(m+n)}\langle\mathrm{Wg}(p), q\rangle d^{\#\left(\pi_{p \cdot \epsilon q}\right)} .
$$

and if let $\mathcal{U}$ be the partition with blocks the cycles of $\gamma$

$$
\begin{aligned}
& \mathrm{E}\left(\operatorname{Tr}\left(O^{\epsilon_{1}} \cdots O^{\epsilon_{m}}\right)\right) \mathrm{E}\left(\operatorname{Tr}\left(O^{\epsilon_{m+1}} \cdots O^{\epsilon_{m+n}}\right)\right. \\
& \quad=\sum_{\substack{p, q \in \mathcal{P}_{2}(m+n) \\
p, q \leq \mathcal{U}}} \operatorname{Wg}(\mathcal{U}, p, q) d^{\#\left(\pi_{p \cdot \epsilon q}\right)} .
\end{aligned}
$$

By the multiplicativity of the coefficient of the term of leading order of $\langle\mathrm{Wg}(p), q\rangle$ we thus have

$$
\begin{aligned}
& \operatorname{cov}\left(\operatorname{Tr}\left(O^{\epsilon_{1}} \cdots O^{\epsilon_{m}}\right), \operatorname{Tr}\left(O^{\epsilon_{m+1}} \cdots O^{\epsilon_{m+n}}\right)\right) \\
& =\sum_{\substack{p, q \in \mathcal{P}_{2}(m+n) \\
p, q \leq \mathcal{U}}}\langle\mathrm{Wg}(p), q\rangle d^{\#\left(\pi_{p \cdot \epsilon}\right)}+\mathrm{O}\left(d^{-(m+n)+\#(p \vee q)+\#\left(\pi_{p \cdot \epsilon}\right)-1}\right)
\end{aligned}
$$

As in the proof of Theorem 36, if $\pi_{p{ }_{\epsilon} q}$ has a singleton $(k)$ then $\epsilon_{k}=$ $-\epsilon_{\gamma(k)}$, which is impossible given our construction of $\epsilon$. Thus $\pi_{p \cdot{ }_{\epsilon} q}$ has no singletons. Hence $\#\left(\pi_{p{ }_{\epsilon} q}\right) \leq(m+n) / 2$. Thus $-(m+n)+\#(p \vee$ $q)+\#\left(\pi_{p \cdot \epsilon}\right) \leq 0$, with equality only if $p=q$ and $\pi_{p \cdot{ }_{\epsilon} p}$ is a pairing. 
Let $(r, s) \in p$. Either $\epsilon_{r}=-\epsilon_{s}$ or $\epsilon_{r}=\epsilon_{s}$. As in the proof of Theorem 38 all cycles of $p$ connect the two cycles of $\gamma$ and hence $|m|=|n|$. Also in the case in which $\epsilon_{r}=-\epsilon_{s}$, we have $\left(\gamma^{-1}(r), \gamma(s)\right) \in p$. There are exactly $|m|$ such $p$ 's. In the case $\epsilon_{r}=\epsilon_{s}$, we have $\left(\gamma^{-1}(r), \gamma^{-1}(s)\right) \in p$. There are exactly $|m|$ such $p$ 's. All together there are $2|m|$ such $p$ 's. By Remark 8 the coefficient of $d^{-n / 2}$ in $\langle\mathrm{Wg}(p), p\rangle$ is 1 . This gives the claimed result.

\section{Vanishing of Higher Cumulants of Traces}

Let $\left\{A_{j}\right\}_{j}$ be a family of $d \times d$ random matrices, containing the identity matrix, with a real second order limit distribution. By this we mean that as $d \rightarrow \infty$

$$
\begin{aligned}
\circ & \operatorname{tr}\left(A_{i_{1}}^{\left(\epsilon_{1}\right)} \cdots A_{i_{n}}^{\left(\epsilon_{n}\right)}\right) \text { converges to } \varphi\left(a_{i_{1}}^{\left(\epsilon_{1}\right)} \cdots a_{i_{n}}^{\left(\epsilon_{n}\right)}\right) \text { for all } i_{1}, \ldots, i_{n} \\
& \text { and all } \epsilon_{1}, \ldots, \epsilon_{n} ; \\
\circ & k_{2}\left(\operatorname{Tr}\left(A_{i_{1}}^{\left(\epsilon_{1}\right)} \cdots A_{i_{m}}^{\left(\epsilon_{m}\right)}\right), \operatorname{Tr}\left(A_{i_{m+1}}^{\left(\epsilon_{m+1}\right)} \cdots A_{i_{m+n}}^{\left(\epsilon_{m+n}\right)}\right)\right) \text { converges to } \\
& \varphi_{2}\left(a_{i_{1}}^{\left(\epsilon_{1}\right)} \cdots a_{i_{m}}^{\left(\epsilon_{m}\right)}, a_{i_{m+1}}^{\left(\epsilon_{m+1}\right)} \cdots a_{i_{m+n}}^{\left(\epsilon_{m+n}\right)}\right) \text { for all } i_{1}, \ldots, i_{m+n} \text { and all } \\
& \epsilon_{1}, \ldots, \epsilon_{m+n} ; \\
\circ & k_{r}\left(\operatorname{Tr}\left(A_{i_{1}}^{\left(\epsilon_{1}\right)} \cdots A_{i_{m_{1}}}^{\left(\epsilon_{m_{1}}\right)}\right), \cdots, \operatorname{Tr}\left(A_{i_{m_{1}+\cdots m_{r}-1}+1}^{\left(\epsilon_{m_{1}+\cdots+m_{r}+1}\right)} \cdots A_{\left.i_{m_{1}+\cdots+m_{r}}\right)}^{\left(\epsilon_{m_{1}+\cdots+m_{r}}\right)}\right)\right) \\
& \text { converges to } 0 \text { for all } r \geq 3, \text { all } i_{1}, \ldots, i_{m_{1}+\cdots+m_{r}} \text { and all } \epsilon_{1}, \ldots, \\
& \epsilon_{m_{1}+\cdots+m_{r}} .
\end{aligned}
$$

Let $O$ be a Haar distributed $d \times d$ random orthogonal matrix whose entries are independent from those of $\left\{A_{j}\right\}_{j}$. In this section we shall show that whenever $X_{1}, \ldots, X_{r}$ be $r$ random variables where each $X_{i}$ is one of the following types:

$$
\left.\begin{array}{l}
\circ X_{i}=\operatorname{Tr}\left(A_{k}\right) \text { for some } k \text {; or } \\
\circ X_{i}=\operatorname{Tr}\left(O^{\epsilon_{1}} A_{j_{1}} \cdots O^{\epsilon_{n}} A_{j_{n}}\right) \text { with } \epsilon_{k} \in\{-1,1\} \text { and such } \\
\quad \text { that if } A_{j_{k}}=I \text { then } \epsilon_{k-1}=\epsilon_{k}, \text { where } \epsilon_{n+1}=\epsilon_{1} .
\end{array}\right\}
$$

The the third and higher cumulants of the $X$ 's will converge to 0 as $d \rightarrow \infty$. This, combined with Theorems 36 and 38 will show that we have asymptotic real second order freeness of the $\left\{A_{j}\right\}_{j}$ and $O$.

For the rest of this section we shall assume that the $\left\{A_{j}\right\}_{j}$ satisfy condition (23) and our goal is to prove the theorem below.

Theorem 41. Suppose that $X_{1}, \ldots, X_{r}$ are of the form (24) and $r \geq 3$, then

$$
\lim _{d \rightarrow \infty} k_{r}\left(X_{1}, \ldots, X_{r}\right)=0
$$


We prove this theorem by proving the following result where we strengthen the hypothesis in (24) by assuming that the non-constant $A$ 's are centred.

i) $X_{i}=\operatorname{Tr}\left(A_{k}\right)$ for some $k$ with $\mathrm{E}\left(\operatorname{Tr}\left(X_{k}\right)\right)=0$; or

ii) $X_{i}=\operatorname{Tr}\left(O^{\epsilon_{1}} A_{j_{1}} \cdots O^{\epsilon_{n}} A_{j_{n}}\right)$ with $\epsilon_{k} \in\{-1,1\}$ and such that either $\mathrm{E}\left(\operatorname{Tr}\left(A_{j_{k}}\right)\right)=0$ or $A_{j_{k}}=I$ and
$\epsilon_{k-1}=\epsilon_{k}$, where $\epsilon_{n+1}=\epsilon_{1}$.

Theorem 42. Suppose that whenever $X_{1}, \ldots, X_{r}$ are of form (26) and $r \geq 3$ then

$$
\lim _{d \rightarrow \infty} k_{r}\left(X_{1}, \ldots, X_{r}\right)=0 .
$$

Proof of Theorem 41 using Theorem 42: We begin by recalling that the cumulant $k_{r}\left(X, \ldots, X_{r}\right)$ will be 0 whenever an $X_{i}$ is constant and $r \geq 2$. Recall also that by our assumption of a second order limit distribution $\mathrm{E}\left(\operatorname{tr}\left(A_{i}\right)\right)$ is a convergent function of $d$ and thus bounded. Thus if $k_{r}\left(X_{1}, \ldots, X_{r}\right) \rightarrow 0$ then so does $\mathrm{E}\left(\operatorname{tr}\left(A_{j}\right)\right) k_{r}\left(X_{1}, \ldots, X_{r}\right)$.

Suppose $X_{i}=\operatorname{Tr}\left(A_{j}\right)$ for some $j$. Let $A_{j}=A_{j}-\mathrm{E}\left(\operatorname{tr}\left(A_{j}\right)\right) I$. Let $c=\mathrm{E}\left(\operatorname{tr}\left(A_{j}\right)\right)$. Then $\mathrm{E}\left(\operatorname{Tr}\left(\AA_{j}\right)\right)=0$ and $A_{j}=\AA_{j}+c I$. Then $k_{r}\left(X_{1}, \ldots, X_{i-1}, c d, X_{i+1}, \ldots, X_{r}\right)=0$ and so

$$
\begin{aligned}
k_{r}\left(X_{1}, \ldots, X_{r}\right) & \\
= & k_{r}\left(X_{1}, \ldots, X_{i-1}, c d, X_{i+1}, \ldots, X_{r}\right) \\
& \quad+k_{r}\left(X_{1}, \ldots, X_{i-1}, \operatorname{Tr}\left(\AA_{j}\right), X_{i+1}, \ldots, X_{r}\right) \\
= & k_{r}\left(X_{1}, \ldots, X_{i-1}, \operatorname{Tr}\left(\AA_{j}\right), X_{i+1}, \ldots, X_{r}\right) .
\end{aligned}
$$

So we may suppose that any $X$ 's of the form $\operatorname{Tr}\left(A_{j}\right)$ are centred.

Next suppose that $X_{i}=\operatorname{Tr}\left(O^{\eta_{1}} A_{j_{1}} \cdots O^{\eta_{s}} A_{j_{s}}\right)$, with each $\eta_{i}= \pm 1$ and whenever $A_{j_{t}}=I$ we have $\eta_{t}=\eta_{t+1}$. For each $i$, we shall write $X_{i}=$ $\operatorname{Tr}\left(O^{\eta_{1}} A_{j_{1}} \cdots O^{\eta_{s}} A_{j_{s}}\right)$ as a linear combination of a constant random variable and terms of the form $\operatorname{Tr}\left(A_{j_{t}}\right)$, or $\operatorname{Tr}\left(O^{\mu_{1}} A_{k_{1}} \cdots O^{\mu_{l}} A_{k_{l}}\right)$ where for each $t$ either $\mathrm{E}\left(\operatorname{Tr}\left(A_{k_{t}}\right)\right)=0$ or $A_{k_{t}}=I$ and $\mu_{t}=\mu_{t+1}$; where $\mu_{l+1}=$ $\mu_{1}$. We then replace $X_{i}$ in $k_{r}\left(X_{1}, \ldots, X_{r}\right)$ by this linear combination and get a sum of cumulants in which all the $A$ 's are of the form (26).

To show that each $X_{i}=\operatorname{Tr}\left(O^{\eta_{1}} A_{j_{1}} \cdots O^{\eta_{s}} A_{j_{s}}\right)$ can be written as such a linear combination we replace for each $t, A_{j_{t}}$ with ${\stackrel{\circ}{j_{t}}}_{1}+\mathrm{E}\left(\operatorname{tr}\left(A_{j_{t}}\right)\right) I$. We then expand this sum. If we have a factor $\mathrm{E}\left(\operatorname{tr}\left(A_{j_{t}}\right)\right) I$, we will get cancellation of cyclically adjacent $O$ 's wherever $\eta_{t}=-\eta_{t+1}$. This might bring two centred $A$ 's next to each other. As the product will not necessarily be such the expectation of the trace is 0 , we repeat the centring process and continue. Since the number of factors decreases 
whenever there is a cancellation, the process terminates with either: an $X_{i}$ of the form $(26, i)$; an $X_{i}$ as in $(26, i i)$; or a constant $X_{i}$ (if all the $O$ 's get cancelled).

Remark 43. To illustrate the previous theorem let us consider the example

$$
k_{3}\left(\operatorname{Tr}\left(O A_{1} O^{-1} A_{2}\right), \operatorname{Tr}\left(O A_{3} O A_{4}\right), \operatorname{Tr}\left(O A_{5} O^{-1} A_{6}\right)\right) .
$$

There are six $A$ 's and we let $A_{i}=\AA_{i}+c_{i} I$ with $c_{i}=\mathrm{E}\left(\operatorname{Tr}\left(A_{i}\right)\right)$. This produces $2^{6}$ terms, some of which are 0 because some of the entries of the cumulant are constant. For example we shall get terms such as

$$
c_{1} c_{3} c_{4} c_{5} k_{3}\left(\operatorname{Tr}\left(\AA_{2}\right), \operatorname{Tr}(O I O I), \operatorname{Tr}\left(\AA_{6}\right)\right) \text {. }
$$

If we started with the example

$$
k_{3}\left(\operatorname{Tr}\left(O A_{1} O^{-1} A_{2}\right), \operatorname{Tr}\left(O A_{3} O^{-1} A_{4}\right), \operatorname{Tr}\left(O A_{5} O^{-1} A_{6}\right)\right) .
$$

then we would also get terms like

$$
c_{1} c_{3} c_{5} k_{3}\left(\operatorname{Tr}\left(\AA_{2}\right), \operatorname{Tr}\left(\AA_{4}\right), \operatorname{Tr}\left(\stackrel{\circ}{A}_{6}\right)\right)
$$

where there no $O$ 's.

Our task now is to prove Theorem 42. We shall recall the moment cumulant relation

$$
\mathrm{E}\left(X_{1} \cdots X_{r}\right)=\sum_{\mathcal{U} \in \mathcal{P}(r)} k_{\mathcal{U}}\left(X_{1}, \ldots, X_{r}\right) .
$$

So to prove something about the cumulants $k_{r}\left(X_{1}, \ldots, X_{r}\right)$ we shall prove something first about $\mathrm{E}\left(X_{1} \cdots X_{r}\right)$ and use this to prove Theorem 42 . We let $\mathcal{P}_{1,2}(n)$ be the partitions of $[n]$ with blocks of size either 1 or 2 .

Theorem 44. Whenever $X_{1}, \ldots, X_{r}$ are of form (26) then

$$
\mathrm{E}\left(X_{1} \cdots X_{r}\right)=\sum_{\mathcal{U} \in \mathcal{P}_{1,2}(r)} k_{\mathcal{U}}\left(X_{1}, \ldots, X_{r}\right)+\mathrm{o}(1) .
$$

Proof of Theorem 42 using Theorem 44.

By Corollary 37 we have that $k_{1}\left(X_{i}\right)=\mathrm{O}(1)$ is $X_{i}$ is of type 26, ii) and $k_{1}\left(X_{i}\right)=0$ if $X_{i}$ is of type (26.i). If $X_{i_{1}}$ and $X_{i_{2}}$ are both of type (26.ii) then by Theorem $38, k_{2}\left(X_{i_{1}}, X_{i_{2}}\right)=\mathrm{O}(1)$. If they are both of type (26. $i$ ), then by assumption (23) we have $k_{2}\left(X_{i_{1}}, X_{i_{2}}\right)=\mathrm{O}(1)$. If $X_{i_{1}}$ is of type (26. $i)$ and $X_{i_{2}}$ is of type (26.ii), then $k_{2}\left(X_{i_{1}}, X_{i_{2}}\right)=\mathrm{E}\left(X_{i_{1}} X_{i_{2}}\right)$, as $\mathrm{E}\left(X_{i_{1}}\right)=0$. Then by Corollary 37, $\mathrm{E}\left(X_{i_{1}} X_{i_{2}}\right)=\mathrm{O}\left(d^{-1}\right)$. So in all cases $k_{1}\left(X_{i_{1}}\right)$ and $k_{2}\left(X_{i_{1}}, X_{i_{2}}\right)$ are of order at most $\mathrm{O}(1)$. 
Now by 28

$$
\begin{aligned}
& k_{3}\left(X_{i_{1}}, X_{i_{2}}, X_{i_{3}}\right) \\
& \quad=\mathrm{E}\left(X_{i_{1}} X_{i_{2}} X_{i_{3}}\right)-\sum_{\mathcal{U} \in \mathcal{P}_{1,2}(3)} k_{\mathcal{U}}\left(X_{i_{1}}, X_{i_{2}}, X_{i_{3}}\right)=\mathrm{o}(1) .
\end{aligned}
$$

Suppose we have shown for $3 \leq s<l$ that $k_{s}\left(X_{i_{1}}, \ldots, X_{i_{s}}\right)=\mathrm{o}(1)$. Then

$$
\begin{aligned}
& \mathrm{E}\left(X_{i_{1}} \cdots X_{i_{l}}\right)-\sum_{\mathcal{U} \in \mathcal{P}_{1,2}(l)} k_{\mathcal{U}}\left(X_{i_{1}}, \ldots, X_{i_{l}}\right) \\
& =k_{l}\left(X_{i_{1}}, \ldots, X_{i_{l}}\right)+\sum_{\mathcal{U} \in \widetilde{\mathcal{P}}_{1,2}(l)} k_{\mathcal{U}}\left(X_{i_{1}}, \ldots, X_{i_{l}}\right)
\end{aligned}
$$

Where $\widetilde{\mathcal{P}}_{1,2}(l)$ is all the partitions in $\mathcal{P}(l)$ except those in $\mathcal{P}_{1,2}(l)$ and $1_{l}$, the partition with only one block. If $\mathcal{U} \in \widetilde{\mathcal{P}}_{1,2}(l)$ then $\mathcal{U}$ has blocks of size 1 or 2 and at least one block of size between 3 and $s$. Since the cumulants from the blocks of order $\mathrm{O}(1)$ and, by our induction hypothesis, all others are of order $\mathrm{O}\left(d^{-1}\right)$, the product $k_{\mathcal{U}}\left(X_{i_{1}}, \ldots, X_{i_{l}}\right)$ is of order $\mathrm{o}(1)$. Hence

$$
k_{l}\left(X_{i_{1}}, \ldots, X_{i_{l}}\right)+\sum_{\mathcal{U} \in \widetilde{\mathcal{P}}_{1,2}(l)} k_{\mathcal{U}}\left(X_{i_{1}}, \ldots, X_{i_{l}}\right)=\mathrm{o}(1)
$$

forces us to conclude that $k_{l}\left(X_{i_{1}}, \ldots, X_{i_{l}}\right)=\mathrm{o}(1)$.

Notation 45. From now on we shall assume that we have positive integers $n_{1}, \ldots, n_{r}$. We let $n=n_{1}+\cdots+n_{r}$. There is $1 \leq r_{0} \leq r$ such that for $r_{0} \leq i \leq r$ we have $n_{i}=1$. We let $\gamma \in S_{n}$ be the permutation with cycles

$$
\begin{gathered}
\left(1, \ldots, n_{1}\right) \cdots\left(n_{1}+\cdots+n_{r_{0}-1}+1, \ldots, n_{1}+\cdots+n_{r_{0}}\right) \\
\quad \times\left(n_{1}+\cdots+n_{r_{0}}+n_{r_{0}+1}\right) \cdots\left(n_{1}+\cdots+n_{r_{0}}+n_{r}\right)
\end{gathered}
$$

If $r_{0}=1$ then $\gamma=e$ is the identity permutation. We shall assume the random variables $X_{i}$ are such that for $1 \leq i \leq r_{0}$

$$
\circ X_{i}=\operatorname{Tr}\left(O^{\epsilon_{n_{1}+\cdots+n_{i-1}+1}} A_{n_{1}+\cdots+n_{i-1}+1} \cdots O^{\epsilon_{n_{1}+\cdots+n_{i}}} A_{n_{1}+\cdots+n_{i}}\right)
$$

where for each $n_{1}+\cdots+n_{i-1}+1 \leq t \leq n_{1}+\cdots+n_{i}$ either $\mathrm{E}\left(\operatorname{Tr}\left(A_{t}\right)\right)=0$ or $A_{t}=I$ and $\epsilon_{t}=\epsilon_{\gamma(t)} ;$

and for $r_{0}<i \leq r$

$$
\circ X_{i}=\operatorname{Tr}\left(A_{n_{1}+\cdots+n_{i}}\right) \text { and } \mathrm{E}\left(X_{i}\right)=0 .
$$

Let $m=n_{1}+\cdots+n_{r_{0}-1}$. If $m$ is odd and positive then $\mathrm{E}\left(X_{1} \cdots X_{r}\right)=0$. So we shall assume that $m$ is even, and possibly 0 . Let $\mathcal{P}_{2}(m, n)$ be the set of partitions of $[n]$ whose restriction to $[\mathrm{m}]$ is a pairing and all of whose other blocks are singletons. In the case $r_{0}=1$ we have $m=0$ 
and the only partition in $\mathcal{P}_{2}(m, n)$ is the one with $n$ blocks of size 1 . We assume that $\epsilon \in \mathbb{Z}_{2}^{n}$ with $\epsilon_{i}=1$ for $i>m$.

Now let $p$ and $q$ be in $\mathcal{P}_{2}(m, n)$. Then $p \delta q \delta$ is a permutation of $[ \pm n]$ whose restriction to $[ \pm m]$ is a pairing and all of whose other cycles are singletons. Now consider $\gamma_{-}^{-1} \delta_{\epsilon} p \delta q \delta \delta_{\epsilon} \gamma$. Its restriction to $[ \pm n] \backslash[ \pm m]$ consists of singletons. Its restriction to $[ \pm m]$ is as in Notation 11, i.e. the cycles occur in pairs $\left\{c, c^{\prime}\right\}$. We obtained a permutation, $\pi_{p_{\epsilon} q}$, of $[m]$ as follows. For each pair we choose one representative, replacing any negative entries by their absolute values. Now we wish to extend this construction to the case where $p, q \in \mathcal{P}_{2}(m, n)$. The cycles in $[ \pm n] \backslash[ \pm m]$ also occur in pairs $(-k)(k)$ (with $k>0$ ) and so we just choose $(k)$ for each of these cycles. Also for $m<k \leq n$ let $\eta_{p \cdot{ }_{\epsilon} q}(k)=1$.

Let $X_{1}, \ldots, X_{r}$ satisfy (26) and let us expand $\mathrm{E}\left(X_{1} \cdots X_{r}\right)$ as follows.

$$
\begin{aligned}
& \mathrm{E}\left(X_{1} \cdots X_{r}\right) \\
& \quad=\sum_{p, q \in \mathcal{P}_{2}(m, n)}\langle\mathrm{Wg}(p), q\rangle \mathrm{E}\left(\operatorname{Tr}_{\left(\pi_{p_{\epsilon} q}, \eta_{p \cdot \epsilon q}\right)}\left(A_{1}, \ldots, A_{n}\right)\right) .
\end{aligned}
$$

We need to find the order of $\mathrm{E}\left(\operatorname{Tr}_{\left(\pi_{p \cdot \epsilon q}, \eta_{p \cdot \epsilon}\right)}\left(A_{1}, \ldots, A_{n}\right)\right)$.

Proposition 46. If $m \geq 2$ and $\left.\pi_{p \cdot{ }_{\epsilon} q}\right|_{[m]}$ is not a pairing then

$$
\mathrm{E}\left(\operatorname{Tr}_{\left(\pi_{p \cdot \epsilon q}, \eta_{p_{\epsilon} q}\right)}\left(A_{1}, \ldots, A_{n}\right)\right)=\mathrm{O}\left(d^{m / 2-1}\right) .
$$

If $\left.\pi_{p \cdot{ }_{\epsilon} q}\right|_{[m]}$ is a pairing or if $m=0$ then

$$
\begin{aligned}
& \mathrm{E}\left(\operatorname{Tr}_{\left(\pi_{p \cdot \epsilon q}, \eta_{p \cdot \epsilon q}\right)}\left(A_{1}, \ldots, A_{m}\right)\right) \\
& =\mathrm{E}_{\left.\pi_{p \cdot \epsilon q}\right|_{[m]}}\left(\operatorname{Tr}_{\left(\left.\pi_{p \cdot \epsilon q}\right|_{[m]},\left.\eta_{p \cdot \epsilon}\right|_{[m]}\right)}\left(A_{1}, \ldots, A_{m}\right)\right) \mathrm{E}\left(\operatorname{Tr}\left(A_{m+1}\right) \cdots \operatorname{Tr}\left(A_{n}\right)\right) \\
& \quad+\mathrm{O}\left(d^{m / 2-1}\right) .
\end{aligned}
$$

Proof. Let follow the notation used in Equation (9). If $\mathcal{U}$ is a partition on $[n]$ and $\pi$ any permutation of $[n]$ we write $\mathrm{E}_{\mathcal{U}}\left(\operatorname{Tr}_{\pi}\left(A_{1}, \ldots, A_{n}\right)\right)$ to be the product $\prod_{i=1}^{k} \mathrm{E}\left(\operatorname{Tr}_{\pi_{i}}\left(A_{1}, \ldots, A_{n}\right)\right)$, where the blocks of $\mathcal{U}$ are $\left\{U_{1}, \ldots, U_{k}\right\}$ and $\pi_{i}=\left.\pi\right|_{U_{i}}$. We likewise let $k_{\mathcal{U}}\left(\operatorname{Tr}_{\pi}\left(A_{1}, \ldots, A_{n}\right)\right)$ be the product of cumulants along the blocks of $\mathcal{U}$, see equation 10 . Recall that we then have the moment-cumulant relation

$$
\begin{aligned}
& \mathrm{E}\left(\operatorname{Tr}_{\left(\pi_{r_{\epsilon} q}, \eta_{p \cdot \epsilon q}\right)}\left(A_{1}, \ldots, A_{n}\right)\right) \\
& =\sum_{\substack{\mathcal{U} \in \mathcal{P}(n) \\
\pi_{p \cdot \epsilon q} \leq \mathcal{U}}} k_{\mathcal{U}}\left(\operatorname{Tr}_{\left(\pi_{p \cdot \epsilon q}, \eta_{p \cdot \epsilon q}\right)}\left(A_{1}, \ldots, A_{n}\right)\right) .
\end{aligned}
$$

By our assumption (23) on the existence of a real second order limit distribution of the $A$ 's we have

$$
k_{\mathcal{U}}\left(\operatorname{Tr}_{\left(\pi_{p \cdot \epsilon q}, \eta_{p \cdot \epsilon q}\right)}\left(A, \ldots, A_{n}\right)\right)=\mathrm{O}\left(d^{u}\right)
$$


where $u$ is the number of blocks of $\mathcal{U}$ that contain only one cycle of $\pi_{p{ }_{\epsilon} q}$. Suppose $k_{\mathcal{U}}\left(\operatorname{Tr}_{\left(\pi_{p \cdot \epsilon}, \eta_{p \cdot \epsilon q}\right)}\left(A, \ldots, A_{n}\right)\right) \neq 0$. Then any block of $\mathcal{U}$ that contains a single cycle of $\pi_{p \cdot{ }_{\epsilon} q}$ must contain a cycle of $\left.\pi_{p \cdot{ }_{\epsilon} q}\right|_{[n]}$, as $\mathrm{E}\left(\operatorname{Tr}\left(A_{k}\right)\right)=0$ for $m<k \leq n$. Thus $u \leq \#\left(\left.\pi_{p \cdot{ }_{\epsilon} q}\right|_{[m]}\right)$. Also recall, from the fourth paragraph of the proof of Theorem 36 , that if $(k)$ is a singleton of $\left.\pi_{p{ }_{\epsilon} q}\right|_{[m]}$ then $\mathrm{E}\left(\operatorname{Tr}\left(A_{k}\right)\right)=0$, and hence $k_{\mathcal{U}}\left(\operatorname{Tr}_{\left(\pi_{p^{\prime} \epsilon q}, \eta_{p \cdot{ }^{*} q}\right)}\left(A_{1}, \ldots\right.\right.$, $\left.\left.A_{n}\right)\right)=0$. So for any block $U$ of $\mathcal{U}$ that contains only one cycle of $\pi_{p{ }_{\epsilon} q}$, $U$ must contain at least two elements. Thus $u \leq m / 2$. We can only have $u=m / 2$ when every block of $\left.\mathcal{U}\right|_{[m]}$ contains one cycle of $\left.\pi_{p{ }{ }_{\epsilon} q}\right|_{[m]}$ and that cycle has two elements, i.e. $\left.\pi_{p \cdot{ }_{\epsilon} q}\right|_{[m]}$ is a pairing. This proves the first claim.

If $\left.\pi_{p \cdot{ }_{\epsilon} q}\right|_{[m]}$ is a pairing then we have just seen that to have $u=m / 2$ we must have $\left.\mathcal{U}\right|_{[m]}=\left.\pi_{p_{\epsilon} q}\right|_{[m]}$. Thus if we only consider $\mathcal{U}$ 's for which $\left.\mathcal{U}\right|_{[m]}=\left.\pi_{p \cdot{ }_{\epsilon} q}\right|_{[m]}$ we have

$$
\begin{aligned}
& \mathrm{E}\left(\operatorname{Tr}_{\left(\pi_{p \cdot \epsilon q}, \eta_{p \cdot \epsilon q}\right)}\left(A_{1}, \ldots, A_{n}\right)\right) \\
& =\mathrm{E}_{\left.\pi_{p \cdot \epsilon q}\right|_{[m]}}\left(\operatorname{Tr}_{\left(\left.\pi_{p \cdot \epsilon q}\right|_{[m]},\left.\eta_{p \cdot \epsilon q}\right|_{[m]}\right)}\left(A_{1}, \ldots, A_{m}\right)\right) \\
& \times \sum_{\mathcal{U}^{\prime} \in \mathcal{P}([m+1, n])} k_{\mathcal{U}^{\prime}}\left(\operatorname{Tr}\left(A_{m+1}\right), \ldots, \operatorname{Tr}\left(A_{m}\right)\right) \\
& =\mathrm{E}_{\left.\pi_{p_{\epsilon} q}\right|_{[m]}}\left(\operatorname{Tr}_{\left(\left.\pi_{p \cdot \epsilon q}\right|_{[m]},\left.\eta_{p \cdot \epsilon q}\right|_{[m]}\right)}\left(A_{1}, \ldots, A_{m}\right)\right) \mathrm{E}\left(\operatorname{Tr}\left(A_{m+1}\right), \ldots, \operatorname{Tr}\left(A_{m}\right)\right)
\end{aligned}
$$

Finally we add back the remaining terms to obtain that

$$
\begin{aligned}
& \mathrm{E}\left(\operatorname{Tr}_{\left(\pi_{p \cdot \epsilon q}, \eta_{p \cdot \epsilon q}\right)}\left(A_{1}, \ldots, A_{n}\right)\right) \\
& =\mathrm{E}_{\left.\pi_{p_{\epsilon} q}\right|_{[m]}}\left(\operatorname{Tr}_{\left(\left.\pi_{p^{*} \epsilon}\right|_{[m]},\left.\eta_{p_{\epsilon} q}\right|_{[m]}\right)}\left(A_{1}, \ldots, A_{m}\right)\right) \mathrm{E}\left(\operatorname{Tr}\left(A_{m+1}\right), \ldots, \operatorname{Tr}\left(A_{m}\right)\right) \\
& +\mathrm{O}\left(d^{m / 2-1}\right) \text {. }
\end{aligned}
$$

Notation 47. Suppose we have $r_{0}, r, m, n$ and $\gamma$ and $\epsilon$ as in Notation 45. Let $\mathcal{A}(\gamma, \epsilon, m, n)$ be the set of partitions $p \in \mathcal{P}_{2}(m, n)$ such that $\left.\pi_{p \cdot{ }_{\epsilon} p}\right|_{[m]}$ is a pairing, the condition being vacuously satisfied when $m=$

0 . For $p \in \mathcal{A}(\gamma, \epsilon, m, n)$ let

$$
\begin{aligned}
& \mathcal{E}_{p}\left(A_{1}, \ldots, A_{n}\right) \\
& =d^{-m / 2} \mathrm{E}_{\left.\pi_{p \cdot \epsilon p}\right|_{[m]}}\left(\operatorname{Tr}_{\left(\left.\pi_{p \cdot \epsilon p}\right|_{[m]},\left.\eta_{p \cdot \epsilon p}\right|_{[m]}\right)}\left(A_{1}, \ldots, A_{m}\right)\right) \\
& \quad \times \mathrm{E}\left(\operatorname{Tr}\left(A_{m+1}\right) \cdots \operatorname{Tr}\left(A_{n}\right)\right) .
\end{aligned}
$$

Corollary 48. Suppose $X_{1}, \ldots, X_{r}$ satisfy (26). Then

$$
\mathrm{E}\left(X_{1} \cdots X_{r}\right)=\sum_{p \in \mathcal{A}(\gamma, \epsilon, m, n)} \mathcal{E}_{p}\left(A_{1}, \ldots, A_{n}\right)+\mathrm{O}\left(d^{-1}\right) .
$$


Proof. When $m=0$ there is nothing to prove. According to Proposition 14

$$
\begin{aligned}
\mathrm{E} & \left(X_{1} \cdots X_{r}\right) \\
& =\sum_{p, q \in \mathcal{P}_{2}(m, n)}\langle\mathrm{Wg}(p), q\rangle \mathrm{E}\left(\operatorname{Tr}_{\left(\pi_{p \cdot \epsilon q}, \eta_{p \cdot \epsilon q}\right)}\left(A_{1}, \ldots, A_{n}\right)\right) .
\end{aligned}
$$

By Proposition 46, if $\left.\pi_{p \cdot{ }_{\epsilon} q}\right|_{[m]}$ is not a pairing we have

$$
\mathrm{E}\left(\operatorname{Tr}_{\left(\pi_{p \cdot \epsilon q}, \eta_{p \cdot \epsilon q}\right)}\left(A_{1}, \ldots, A_{n}\right)\right)=\mathrm{O}\left(d^{m / 2-1}\right)
$$

and $\langle\mathrm{Wg}(p), q\rangle=\mathrm{O}\left(d^{-n+\#(p \vee q)}\right)$. So

$$
\langle\mathrm{Wg}(p), q\rangle \mathrm{E}\left(\operatorname{Tr}_{\left(\pi_{p \cdot \epsilon q}, \eta_{p \cdot \epsilon q}\right)}\left(A_{1}, \ldots, A_{n}\right)\right)=\mathrm{O}\left(d^{-1}\right) .
$$

Also if $\#(p \vee q)<n / 2$ (i.e. $p \neq q)$ we get the same conclusion. When $p=q$ and $\left.\pi_{p \cdot{ }_{\epsilon} p}\right|_{[m]}$ is a pairing, then $p \in \mathcal{A}(\gamma, \epsilon, m, n)$ and

$$
\langle\mathrm{Wg}(p), q\rangle \mathrm{E}\left(\operatorname{Tr}_{\left(\pi_{p \cdot \epsilon}, \eta_{p \cdot \epsilon q}\right)}\left(A_{1}, \ldots, A_{n}\right)\right)=\mathcal{E}_{p}\left(A_{1}, \ldots, A_{n}\right)+\mathrm{O}\left(d^{-1}\right)
$$

because $\langle\mathrm{Wg}(p), p\rangle=d^{-m / 2}+\mathrm{O}\left(d^{-m / 2-1}\right)$.

Proof of Theorem 44: To prove the theorem we show that

$$
\sum_{\mathcal{U} \in \mathcal{P}_{2}(r)} k_{\mathcal{U}}\left(X_{1}, \ldots, X_{r}\right)=\sum_{p \in \mathcal{A}(\gamma, \epsilon, m, n)} \mathcal{E}_{p}\left(A_{1}, \ldots, A_{n}\right)+\mathrm{o}(1)
$$

and then apply Corollary 48 . We saw in the proof of Theorem 42 that if $X_{i_{1}}$ is of type (26, $\left.i\right)$ and $X_{i_{2}}$ is of type (26. ii) then $k_{2}\left(X_{i_{1}}, X_{i_{2}}\right)=$ $\mathrm{O}\left(d^{-1}\right)$, so on the left hand side of 30 we only have to consider $\mathcal{U}$ 's for which each block is either contained in $[m]$ or in $[m+1, n]$. Thus

$$
\begin{aligned}
& \sum_{\mathcal{U} \in \mathcal{P}_{2}(r)} k_{\mathcal{U}}\left(X_{1}, \ldots, X_{r}\right) \\
& =\sum_{\mathcal{U} \in \mathcal{P}_{2}\left(r_{0}\right)} k_{\mathcal{U}}\left(X_{1}, \ldots, X_{r_{0}}\right) \sum_{\mathcal{V} \in \mathcal{P}_{2}\left(\left[r_{0}+1, r\right]\right)} k_{\mathcal{V}}\left(X_{r_{0}+1}, \ldots, X_{r}\right)+\mathrm{o}(1) .
\end{aligned}
$$

By assumption (23) we have

$$
\sum_{\mathcal{V} \in \mathcal{P}_{1,2}\left(\left[r_{0}+1, r\right]\right)} k_{\mathcal{V}}\left(X_{r_{0}+1}, \ldots, X_{r}\right)=\mathrm{E}\left(X_{m+1} \cdots X_{n}\right)+\mathrm{o}(1)
$$

because cumulants corresponding to blocks of size three or larger are $\mathrm{o}(1)$ and cumulants corresponding to blocks of size two are $\mathrm{O}(1)$ and cumulants corresponding to blocks of size one are 0 .

Let us next show that

$$
\sum_{\mathcal{U} \in \mathcal{P}_{1,2}\left(r_{0}\right)} k_{\mathcal{U}}\left(X_{1}, \ldots, X_{r_{0}}\right)
$$




$$
=d^{-m / 2} \sum_{\substack{p \in \mathcal{P}_{2}(m) \\ \pi_{p \cdot \epsilon p} \text { a pairing }}} \mathrm{E}_{\pi_{p \cdot \epsilon p}}\left(\operatorname{Tr}_{\left(\pi_{p \cdot \epsilon}, \eta_{p \cdot \epsilon p}\right)}\left(A_{1}, \ldots, A_{m}\right)\right)+\mathrm{O}\left(d^{-1}\right) .
$$

If we multiply these last two equations we get equation as

$$
\begin{aligned}
& \mathcal{E}_{p}\left(A_{1}, \ldots, A_{n}\right) \\
& \quad=d^{-m / 2} \mathrm{E}_{\pi_{p \cdot \epsilon p}}\left(\operatorname{Tr}_{\left(\pi_{p \cdot \epsilon p}, \eta_{p \cdot \epsilon p}\right)}\left(A_{1}, \ldots, A_{m}\right)\right) \mathrm{E}\left(X_{m+1} \cdots X_{n}\right) .
\end{aligned}
$$

To prove (31) we use $(12)$ and (18). They say that a first and second cumulant of $X$ 's if type (26. ii) can be written, up to terms of order $\mathrm{O}\left(d^{-1}\right)$, as sums over pairings $p$ in unions of intervals of $\gamma$ for which $\pi_{p \cdot{ }_{\epsilon} p}$ is a pairing. Moreover by Corollary 22 if $p \in \mathcal{P}_{2}(m)$ is a pairing and $\pi_{p \cdot{ }_{\epsilon} p}$ is also a pairing then at most two cycles of $\gamma$ can be contained in any block of $p \vee \gamma$.

Let $p \in \mathcal{P}_{2}(m)$ be a pairing such that $\pi_{p \cdot{ }_{\epsilon} p}$ is a pairing. The partition $p \vee \gamma$ determines a partition $\mathcal{U}_{p} \in \mathcal{P}\left(r_{0}\right)$ of the cycles of $\gamma$. By Corollary 22. $\mathcal{U}_{p} \in \mathcal{P}_{1,2}\left(r_{0}\right)$. Thus we can write

$$
\begin{gathered}
d^{-m / 2} \sum_{p \in \mathcal{A}(\gamma, \epsilon, m)} \mathrm{E}_{\pi_{p \cdot \epsilon p}}\left(\operatorname{Tr}_{\left(\pi_{p \cdot \epsilon}, \eta_{p \cdot \epsilon p}\right)}\left(A_{1}, \ldots, A_{m}\right)\right) \\
=\sum_{\mathcal{U} \in \mathcal{P}_{1,2}\left(r_{0}\right)} d^{-m / 2} \sum_{\substack{p \in \mathcal{A}(\gamma, \epsilon, m) \\
\mathcal{U}_{p}=\mathcal{U}}} \mathrm{E}_{\pi_{p \cdot \epsilon p}}\left(\operatorname{Tr}_{\left(\pi_{p \cdot \epsilon p}, \eta_{p \cdot \epsilon}\right)}\left(A_{1}, \ldots, A_{m}\right)\right) .
\end{gathered}
$$

So to prove (31) it suffices to prove that for $\mathcal{U} \in \mathcal{P}_{1,2}\left(r_{0}\right)$

$$
\begin{aligned}
& k_{\mathcal{U}}\left(X_{1}, \ldots, X_{r_{0}}\right) \\
& =\sum_{\substack{p \in \mathcal{A}(\gamma, \epsilon, m) \\
\mathcal{U}_{p}=\mathcal{U}}} \mathrm{E}_{\pi_{p \cdot \epsilon p}}\left(\operatorname{tr}_{\left(\pi_{p \cdot \epsilon}, \eta_{p \cdot \epsilon p}\right)}\left(A_{1}, \ldots, A_{m}\right)\right)+\mathrm{O}\left(d^{-1}\right) .
\end{aligned}
$$

Now $k_{\mathcal{U}}\left(X_{1}, \ldots, X_{r_{0}}\right)$ is a product of first and second cumulants. For each first cumulant, $\mathrm{E}\left(X_{j}\right)$, we apply equation 12 to write

$$
\mathrm{E}\left(X_{j}\right)=d^{-s / 2} \sum_{p} \mathrm{E}_{\pi_{p \cdot \epsilon} p}\left(\operatorname{Tr}_{\left(\pi_{p \cdot \epsilon p}, \eta_{p \cdot \epsilon}\right)}\left(A_{i_{1}}, \ldots, A_{i_{s}}\right)\right)+\mathrm{O}\left(d^{-1}\right)
$$

with $p$ running over pairings of the corresponding cycle $\left(i_{1}, \ldots, i_{s}\right)$ of $\gamma$ such that $\pi_{p \cdot{ }_{\epsilon} p}$ is a pairing.

For each second cumulant $k_{2}\left(X_{k}, X_{l}\right)$ we apply equation (18) to write

$$
\operatorname{cov}\left(X_{k}, X_{l}\right)=d^{-t / 2} \sum_{p} \mathrm{E}_{\pi_{p \cdot \epsilon p}}\left(\operatorname{Tr}_{\left(\pi_{p \cdot \epsilon p}, \eta_{p \cdot \epsilon p}\right)}\left(A_{j_{1}}, \ldots, A_{j_{t}}\right)\right)+\mathrm{O}\left(d^{-1}\right)
$$

with $p$ running over pairings that connect the corresponding union $\left(j_{1}, \ldots, j_{t}\right)$ of two cycles of $\gamma$ such that $\pi_{p \cdot{ }_{\epsilon} p}$ is a pairing. Taking the product of these equations gives us 32 . 


\section{Main Results on Asymptotic Real Second Order Freeness}

In this section we will present some consequences of Theorems 36 , 38 and 41 .

Theorem 49. The ensemble of Haar distributed orthogonal random matrices has a real second order limit distribution.

Proof. Corollaries 10 and 40 show that an ensemble of Haar orthogonal matrices has convergent moments $\left\{\mathrm{E}\left(\operatorname{tr}\left(O^{m}\right)\right)\right\}_{d}$ and convergent fluctuation moments $\left\{k_{2}\left(\operatorname{Tr}\left(O^{m}\right), \operatorname{Tr}\left(O^{n}\right)\right)\right\}_{d}$. A particular example of Theorem 41 is the case when we have $k_{r}\left(\operatorname{Tr}\left(O^{m_{1}}\right), \ldots, \operatorname{Tr}\left(O^{m_{r}}\right)\right)$ for some non-zero integers $m_{1}, \ldots, m_{r}$. Together these results then show that an ensemble of Haar orthogonal random matrices has a real second order limit distribution.

Theorem 50. Suppose $\left\{A_{i}\right\}_{i}$ is an ensemble of random matrices with a real second order limit distribution and $O$ is an ensemble of Haar distributed orthogonal random matrices. If the entries of $\left\{A_{i}\right\}_{i}$ are independent from those of $O$, then $\left\{A_{i}\right\}_{i}$ and $O$ are asymptotically real second order free.

Proof. This is a consequence of Theorems 36,38 and 41.

Theorem 51. Let $O_{1}, \ldots, O_{s}$ be independent Haar distributed orthogonal random matrices. Then $O_{1}, \ldots, O_{s}$ are asymptotically real second order free.

Proof. A single $O$ has a real second order limit distribution by Theorem 49. By Theorem 50, $O_{1}$ and $O_{2}$ are asymptotically real second order free. Again by Theorem $50\left\{O_{1}, O_{2}\right\}$ and $O_{3}$ are asymptotically real second order free. By Proposition 29, $\mathrm{O}_{1}, \mathrm{O}_{2}$, and $\mathrm{O}_{3}$ are asymptotically real second order free. Then we can proceed by induction.

Proposition 52. Suppose $\left\{A_{i}\right\}_{i}$ and $\left\{B_{j}\right\}_{j}$ are two independent families of $d \times d$ random matrices, each having a real second order limit distribution, and suppose that $O$ is a $d \times d$ Haar orthogonal matrix independent from $\left\{A_{i}\right\}_{i} \cup\left\{B_{j}\right\}_{j}$. Then $\left\{B_{j}\right\}_{l}$ and $\left\{O A_{i} O^{-1}\right\}_{i}$ are asymptotically real second order free.

Proof. We do not know that $\left\{A_{i}\right\}_{i} \cup\left\{B_{j}\right\}_{j}$ has a real second order limit distribution so we cannot directly apply Theorems 36, 38 and 41. We shall argue that because of the special nature of the words we are considering, i.e. $O A_{i_{1}} O^{-1} B_{j_{1}} O A_{i_{2}} O^{-1} B_{j_{2}} \cdots O A_{i_{n}} O^{-1} B_{j_{n}}$, the proofs can be modified so that we only need the independence of $\left\{A_{i}\right\}_{i}$ and $\left\{B_{j}\right\}_{j}$ and the fact that the exponents of the $O$ 's alternate in sign. 
Consider the expression

$$
\mathrm{E}\left(\operatorname{Tr}_{\left(\pi_{p \cdot \epsilon q}, \eta_{p^{\prime} \epsilon}\right)}\left(Y_{1}, \ldots, Y_{n}\right)\right)
$$

appearing in the statement of Proposition 12. If we write

$$
\operatorname{Tr}_{\left(\pi_{p \cdot \epsilon q}, \eta_{p_{\epsilon} q}\right)}\left(Y_{1}, \ldots, Y_{n}\right)=\operatorname{Tr}\left(Z_{1}\right) \cdots \operatorname{Tr}\left(Z_{k}\right)
$$

as a product along the cycles $c_{1} \cdots c_{k}$ os $\pi_{p \cdot{ }_{\epsilon} q}$, then the existence of a real second order limit distribution was used to conclude that

$$
\begin{gathered}
d^{-1} k_{1}\left(\operatorname{Tr}\left(Z_{i}\right)\right) \text { converges, } \\
k_{2}\left(\operatorname{Tr}\left(Z_{i}\right), \operatorname{Tr}\left(Z_{j}\right)\right) \text { converges, and } \\
k_{r}\left(\operatorname{Tr}\left(Z_{i_{1}}\right), \ldots, \operatorname{Tr}\left(Z_{i_{r}}\right)\right)=\mathrm{o}(1) \text { for } r \geq 3 .
\end{gathered}
$$

This was all we needed to prove Theorems 36, 38 and 41. We shall show that we still have these three properties even though we do not assume that $\left\{A_{i}\right\}_{i} \cup\left\{B_{j}\right\}_{j}$ has a real second order limit distribution.

So let $n_{1}, n_{2}, \ldots, n_{r}$ be even positive integers and $n=n_{1}+\cdots+n_{r}$. Let

$\gamma=\left(1, \ldots, n_{1}\right)\left(n_{1}+1, \ldots, n_{1}+n_{2}\right) \cdots\left(n_{1}+\cdots+n_{r-1}+1, \ldots, n_{1}+\cdots n_{r}\right)$

be the permutation in $S_{n}$ with the cycle decomposition given above. Let $Y_{1}, Y_{3}, \ldots, Y_{n-1}$ be polynomials in $\left\{A_{i}\right\}_{i}$ and $Y_{2}, Y_{4}, \ldots, Y_{n}$ be polynomials in $\left\{B_{j}\right\}_{j}$. By Proposition 12 we have

$$
\begin{aligned}
& \mathrm{E}\left(\operatorname{Tr}_{\gamma}\left(O Y_{1}, O^{-1} Y_{2}, \ldots, O Y_{n-1}, O^{-1} Y_{n}\right)\right) \\
= & \sum_{p, q \in \mathcal{P}_{2}(n)}\langle\mathrm{Wg}(p), q\rangle \mathrm{E}\left(\operatorname{Tr}_{\left(\pi_{p \cdot \epsilon q}, \eta_{p \cdot \epsilon q}\right)}\left(Y_{1}, \ldots, Y_{n}\right) .\right.
\end{aligned}
$$

Now by Lemma 13

$$
\mathrm{E}\left(\operatorname{Tr}_{\left(\pi_{p_{\epsilon}, q}, \eta_{p_{\epsilon} q}\right)}\left(Y_{1}, \ldots, Y_{n}\right)=\mathrm{E}\left(\operatorname{Tr}\left(Z_{1}\right) \cdots \operatorname{Tr}\left(Z_{k}\right)\right)\right.
$$

where each $Z_{i}$ is a polynomial in either $\left\{A_{i}\right\}_{i}$ or in $\left\{B_{j}\right\}_{j}$. In fact we may suppose that $Z_{1}, \ldots, Z_{l}$ are polynomials in $\left\{A_{i}\right\}_{i}$ and $Z_{l+1}, \ldots, Z_{k}$ are polynomials in $\left\{B_{j}\right\}_{j}$. Then we have

$$
\begin{aligned}
& \mathrm{E}\left(\operatorname{Tr}_{\gamma}\left(O Y_{1}, O^{-1} Y_{2}, \ldots, O Y_{n-1}, O^{-1} Y_{n}\right)\right) \\
&=\sum_{p, q \in \mathcal{P}_{2}(n)}\langle\mathrm{Wg}(p), q\rangle \mathrm{E}\left(\operatorname{Tr}\left(Z_{1}\right) \cdots \operatorname{Tr}\left(Z_{l}\right)\right) \\
& \times \mathrm{E}\left(\operatorname{Tr}\left(Z_{l+1}\right) \cdots \operatorname{Tr}\left(Z_{k}\right)\right)
\end{aligned}
$$

by the independence of the $\left\{A_{i}\right\}_{i}$ and the $\left\{B_{j}\right\}_{j}$. This means that as far as the asymptotic behaviour of $\mathrm{E}\left(\operatorname{Tr}_{\left(\pi_{p \cdot \epsilon q}, \eta_{p \cdot \epsilon q}\right)}\left(Y_{1}, \ldots, Y_{n}\right)\right)$ is concerned we may assume that $\left\{A_{i}\right\}_{i} \cup\left\{B_{j}\right\}_{j}$ does have a real second order limit distribution. Now having cleared this hurdle we have by the proof of Theorem 36 that $\left\{O A_{i} O^{-1}\right\}_{i}$ and $\left\{B_{j}\right\}_{j}$ are first order 
free. Likewise, the proof of Theorem 41 can be applied to conclude that all third and higher cumulants of traces of products of $O A_{i} O^{-1}$ 's and $B_{j}$ 's are of order o(1) as $d \rightarrow \infty$. We shall conclude the proof by showing that Theorem 38 and equation (33) will give us condition (ii) of Definition 25.

So let us consider centred random matrices $X_{1}, \ldots, X_{m}$ and $Y_{1}, \ldots Y_{n}$ where $X_{1}, X_{3}, \ldots X_{m-1}$ and $Y_{1}, Y_{3}, \ldots Y_{n-1}$ are polynomials in $\left\{A_{i}\right\}_{i}$ and $X_{2}, X_{2}, \ldots, X_{m}$ and $Y_{2}, Y_{4}, \ldots, Y_{n}$ are polynomials in $\left\{B_{j}\right\}_{j}$. Let the second order limit distribution of $X_{1}, \ldots, X_{m}$ and $Y_{1}, \ldots, Y_{n}$ be given by $x_{1}, \ldots, x_{m}$ and $y_{1}, \ldots, y_{n}$ respectively.

By equation (14) we have for $m=n$

$$
\begin{aligned}
& \lim _{d \rightarrow \infty} \operatorname{cov}\left(\operatorname{Tr}\left(O X_{1} O^{-1} X_{2} \cdots O^{-1} X_{m}\right), \operatorname{Tr}\left(O Y_{1} \cdots O^{-1} Y_{n}\right)\right) \\
& =\sum_{r=1}^{m}\left\{\prod_{i=1}^{m} \varphi\left(x_{i} y_{r-i}\right) \delta_{\epsilon_{i},-\epsilon_{\gamma}-i+1(m+r)}+\prod_{i=1}^{m} \varphi\left(x_{i} y_{r+i}^{t}\right) \delta_{\epsilon_{i}, \epsilon_{\gamma^{i}(m+r)}}\right\}
\end{aligned}
$$

Since $\epsilon_{i}=(-1)^{i}$, we have both $\delta_{\epsilon_{i},-\epsilon_{\gamma^{-i+1}(m+r)}}=1$ and $\delta_{\epsilon_{i}, \epsilon_{\gamma^{i}(m+r)}}=1$ only when $r$ is even; see Figure 5. Thus

$$
\begin{array}{r}
\sum_{r=1}^{m}\left\{\prod_{i=1}^{m} \varphi\left(x_{i} y_{r-i}\right) \delta_{\epsilon_{i},-\epsilon_{\gamma^{-i+1}(m+r)}}+\prod_{i=1}^{m} \varphi\left(x_{i} y_{r+i}^{t}\right) \delta_{\epsilon_{i}, \epsilon_{\gamma^{i}(m+r)}}\right\} \\
=\sum_{r=1}^{m / 2}\left\{\prod_{i=1}^{m} \varphi\left(x_{i} y_{2 r-i}\right)+\prod_{i=1}^{m} \varphi\left(x_{i} y_{2 r+i}^{t}\right)\right\}
\end{array}
$$

For $i$ odd we write $\varphi\left(x_{i} y_{2 r-i}\right)=\varphi\left(o x_{i} o^{-1} o y_{2 r-i} o^{-1}\right)$. Then

$$
\begin{aligned}
\sum_{r=1}^{m / 2}\left\{\prod_{i=1}^{m} \varphi\left(x_{i} y_{2 r-i}\right)\right. & \left.+\prod_{i=1}^{m} \varphi\left(x_{i} y_{2 r+i}^{t}\right)\right\} \\
= & \sum_{r=1}^{m / 2}\left\{\prod_{i=1}^{m / 2} \varphi\left(\left(o x_{2 i-1} o^{-1}\right)\left(o y_{2 r-(2 i-1)} o^{-1}\right)\right) \varphi\left(x_{2 i} y_{2 r-2 i}\right)\right. \\
& \left.+\prod_{i=1}^{m / 2} \varphi\left(\left(o x_{2 i-1} o^{-1}\right)\left(o y_{2 r+2 i-1} O\right)^{t}\right) \varphi\left(x_{2 i} y_{2 r+2 i}^{t}\right)\right\}
\end{aligned}
$$

This shows that condition (ii) of Definition 25 is satisfied.

Definition 53. A random matrix is said to be invariant under conjugation by an orthogonal matrix if the joint distribution of the entries is invariant under conjugation by an orthogonal matrix. So if we let $A$ 

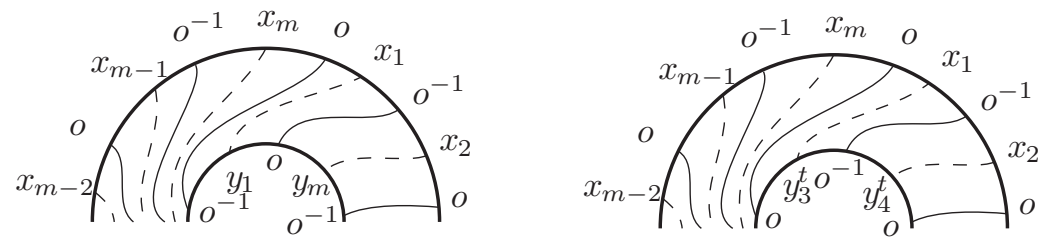

FiguRE 5. When $\epsilon_{i}=(-1)^{i}$ the only spoke diagrams that make a contribution are those where we connect an $o$ to an $o^{-1}$. This means we can only connect an $a$ to a $b$ if the indices have the same parity. This is what we see in equation (34).

be a random matrix, $O$ be a orthogonal matrix and $B=O A O^{-1}$ then we mean that for every $i_{1}, \ldots, i_{n}, i_{-1}, \ldots, i_{-n}$ we have

$$
\mathrm{E}\left(a_{i_{1} i_{-1}} \cdots a_{i_{n} i_{-n}}\right)=\mathrm{E}\left(b_{i_{1} i_{-1}} \cdots b_{i_{n} i_{-n}}\right) .
$$

Many standard examples of random matrices are invariant under conjugation by a unitary or orthogonal matrix. In particular, real Wishart matrices, the Gaussian orthogonal ensemble, Ginibre matrices, and orthogonal matrices are all invariant under conjugation by an orthogonal matrix. In $\left[\mathrm{R}_{1}, \mathrm{R}_{2}\right]$, Redelmeier these were shown to have real second order limit distributions and so satisfy the hypothesis of our theorem below.

Theorem 54. Suppose that $\left\{A_{i}\right\}_{i}$ and $\left\{B_{j}\right\}_{j}$ are two independent families of random matrices, each with real second order limit distribution. Suppose also that the family $\left\{A_{i}\right\}_{i}$ is invariant under conjugation by an orthogonal matrix. Then $\left\{A_{i}\right\}_{i}$ and $\left\{B_{j}\right\}_{j}$ are asymptotically real second order free.

Proof. Since the joint distribution of the entries of $A_{i}$ and $O A_{i} O^{-1}$ are the same we may replace $\left\{A_{i}\right\}_{i}$ by $\left\{O A_{i} O^{-1}\right\}_{i}$ and then apply Proposition 52 .

\section{Concluding Remark}

Let us consider $\left\{A_{i}\right\}_{i}$ and $\left\{B_{l}\right\}_{l}$ two independent ensembles of random matrices, each with a real second order limit distribution and suppose that the ensemble $\left\{A_{i}\right\}_{i}$ is invariant under a conjugation by a unitary matrix. In [MŚS] it is shown that $\left\{A_{i}\right\}_{i}$ and $\left\{B_{l}\right\}_{l}$ are asymptotically complex second order free (see [MŚS., Corollary 3.16). Since orthogonal matrices are also unitary, Theorem 54 implies that $\left\{A_{i}\right\}_{i}$ and $\left\{B_{l}\right\}_{l}$ are asymptotically both real and complex second order free. 
In particular, the second term on the right-hand side of equation (6) must vanish. In consequence, for $A_{1}, A_{2} \in\left\{A_{i}\right\}_{i}$ we have that

$$
\lim _{d \rightarrow \infty} \operatorname{tr}\left(A_{1} A_{2}^{t}\right)=0
$$

The connection between ensembles of random matrices which are invariant under a conjugation with a unitary and real second order freeness goes deeper than this and is investigated in the subsequent paper $\left[\mathrm{MP}_{2}\right]$ in which we show that unitarily invariant ensembles are asymptotically free from their transposes.

\section{REFERENCES}

[AGZ] G. Anderson, A. Guionnet, and O. Zeitouni, An introduction to random matrices, Cambridge University Press, 2010.

[Az] G. Anderson and O. Zeitouni, A CLT for a band matrix model, Probab. Theory Related Fields 134 (2006), 283-338.

[BS] Z. D. Bai and J. W. Silverstein, CLT for linear spectral statistics of largedimensional sample covariance matrices. Ann. Probab. 32 (2004), 553-605.

[C] B. Collins: Moments and cumulants of polynomial random variables on unitary groups, the Itzykson-Zuber integral, and free probability. Int. Math. Res. Not., (17) (2003), 953-982.

[CMśs] B. Collins, J. A. Mingo, P. Śniady, and R. Speicher, Second Order Freeness and Fluctuations of Random Matrices: III. Higher Order Freeness and Free Cumulants, Documenta Math., 12 (2007), 1-70.

[Cs] B. Collins and P. Śniady, Integration with Respect to the Haar Measure on Unitary, Orthogonal, and Symplectic Group, Comm. Math. Phy., 264 (2006), 773-795.

[FMP] J. B. French, P. A. Mello, and A. Pandey, Statistical properties of manyparticle spectra. II. Two-point correlations and fluctuations. Ann. Physics 113 (1978), 277-293.

[J] K. Johansson, On fluctuations of eigenvalues of random Hermitian matrices, Duke Math. J., 91 (1998), 151-204.

[K] C. King. Two-dimensional Potts models and annular partitions, J. Statist. Phy., 96 (1999), 1071-1089.

[KKP] A. Khorunzhy, B. Khoruzhenko, and L. Pastur, On the $1 / N$ corrections to the Green functions of random matrices with independent entries. J. Phys. A 28 (1995), L31-L35.

[LZ] S. K. Lando and A. K. Zvonkin, Graphs on surfaces and their applications, Springer-Verlag, 2004.

[LS] V. P. Leonov and A. N. Shiryaev, On a method of semi-invariants, Theory of Probability and its Applications, 4 (1959), 319-329.

$\left[\mathrm{MP}_{1}\right]$ V. A. Marčenko and L. A. Pastur, Distribution of eigenvalues in certain sets of random matrices, (Russian) Mat. Sb. (N.S.) 72 (114) (1967), 507536.

[MN] J. A. Mingo and A. Nica, Annular noncrossing permutations and partitions, and second-order asymptotics for random matrices, Int. Math. Res. Not., 28 (2004), 1413-1460. 
$\left[\mathrm{MP}_{2}\right]$ J. A. Mingo, M. Popa, On the Relation between the Complex and Real Second order Free Independence, preprint.

[Ms] J. A. Mingo and R. Speicher: Second Order Freeness and Fluctuations of Random Matrices: I. Gaussian and Wishart matrices and Cyclic Fock spaces. J. Funct. Anal., 235, (2006), 226-270.

[MŚs] J. A. Mingo, P. Śniady, and R. Speicher, Second order freeness and fluctuations of random matrices: II. Unitary random matrices. Adv. in Math. 209 (2007), 212-240.

[MST] J. A. Mingo, R. Speicher, and E. Tan, Second Order Cumulants of Products, Trans. Amer. Math. Soc., 361, (2009), 4751-4781.

[Ns] A. Nica and R. Speicher, Lectures on the Combinatorics of Free Probability, Cambridge Univ. Press, 2006.

$\left[\mathrm{R}_{1}\right] \quad$ C. E. I. Redelmeier, Genus expansion for real Wishart matrices, J. Theoret. Probab. 24 (2011), 1044-1062.

$\left[\mathrm{R}_{2}\right] \quad$ C. E. I. Redelmeier, Real second-order freeness and the asymptotic real second-order freeness of several real matrix ensembles, I.M.R.N. (to appear).

[s] R. Speicher: Multiplicative functions on the lattice of noncrossing partitions and free convolution. Math. Ann., 298 (1994), 611-628.

$\left[\mathrm{v}_{1}\right] \quad$ D.-V. Voiculescu, Limit laws for random matrices and free products, Invent. Math. 104 (1991), 201-220.

$\left[\mathrm{v}_{2}\right]$ D.-V. Voiculescu, A strengthened asymptotic freeness result for random matrices with applications to free entropy, Internat. Math. Res. Notices, 1998, no. 1, 41-63.

[vdn] D. V. Voiculescu, K. Dykema, and A. Nica, Free Random variables, Amer. Math. Soc., 1992.

[w] E. P. Wigner, Characteristic vectors of bordered matrices with infinite dimensions, Ann. of Math. 62 (1955), 548564.

प

Department of Mathematics and Statistics, Queen's University, Jeffery Hall, Kingston, Ontario, K7L 3N6, Canada

E-mail address: mingo@mast.queensu.ca

Department of Mathematics and Statistics, Queen's University, Jeffery Hall, Kingston, Ontario, K7L 3N6, Canada, and

Institute of Mathematics "Simion Stoilow" of the Romanian Academy, P.O. Box 1-764, Bucharest, RO-70700, Romania

E-mail address: popa@mast.queensu.ca 